\title{
Nanomaterials for Neural Interfaces
}

\author{
By Nicholas A. Kotov,* Jessica O. Winter,* Isaac P. Clements, Edward Jan, \\ Brian P. Timko, Stéphane Campidelli, Smita Pathak, Andrea Mazzatenta, \\ Charles M. Lieber,* Maurizio Prato,* Ravi V. Bellamkonda,* Gabriel A. Silva,* \\ Nadine Wong Shi Kam, Fernando Patolsky, and Laura Ballerini
}

This review focuses on the application of nanomaterials for neural interfacing. The junction between nanotechnology and neural tissues can be particularly worthy of scientific attention for several reasons: (i) Neural cells are electroactive, and the electronic properties of nanostructures can be tailored to match the charge transport requirements of electrical cellular interfacing. (ii) The unique mechanical and chemical properties of nanomaterials are critical for integration with neural tissue as long-term implants. (iii) Solutions to many critical problems in neural biology/medicine are limited by the availability of specialized materials. (iv) Neuronal stimulation is needed for a variety of common and severe health problems. This confluence of need, accumulated expertise, and potential impact on the well-being of people suggests the potential of nanomaterials to revolutionize the field of neural interfacing. In this review, we begin with foundational topics, such as the current status of neural electrode (NE) technology, the key challenges facing the practical utilization of NEs, and the potential advantages of nanostructures as components of chronic implants. After that the detailed account of toxicology and biocompatibility of nanomaterials in respect to neural tissues is given. Next, we cover a variety of specific applications of nanoengineered devices, including drug delivery, imaging, topographic patterning, electrode design, nanoscale transistors for high-resolution neural interfacing, and photoactivated interfaces. We also critically evaluate the specific properties of particular nanomaterials-including nanoparticles, nanowires, and carbon nanotubes-that can be taken advantage of in neuroprosthetic devices. The most promising future areas of research and practical device engineering are discussed as a conclusion to the review.

\section{Introduction}

The field of nanomaterials offers to have a particularly high impact on medicine ${ }^{[1-4]}$ It is thus appropriate to assess the current state and projected future role of nanotechnology within relevant areas of biomedical science and engineering. Interfacing with neural tissues, for example, is an especially challenging problem that is unsolved by current technologies. There exists a wide range of health problems that can be treated by stimulation of the nervous system, including hearing loss, chronic pain, incontinence, obesity, and diabetes. Some of these problems, such as paralysis or retinal degeneration, are exceptionally hard to treat. At the same time, the set of medical devices and treatments available to a practitioner in this field for evaluating and treating disorders related to neural tissues is especially limited. This situation is caused in part by the poor accessibility of most of the nervous system and by insufficient development of methods to intercept and alter the transmission of neural signals. ${ }^{[3]}$ In the larger sense, interfacing with neural tissue also involves the tasks of its monitoring and drug delivery, which are also often more challenging than for other tissues. The combination of these
[*] Prof. N. A. Kotov, E. Jan, Dr. N. W. S. Kam

Departments of Chemical Engineering, Biomedical Engineering, and

Materials Science and Engineering, University of Michigan

Ann Arbor, MI 48109 (USA)

E-mail: kotov@umich.edu

Prof. J. O. Winter

Department of Chemical Engineering and Biomedical Engineering

Ohio State University, Columbus, OH (USA)

E-mail: winter.63@osu.edu

Prof. C. M. Lieber, Dr. B. P. Timko, Prof. F. Patolsky

Department of Chemistry and Biochemistry

Harvard University, Cambridge, MA (USA)

E-mail: cml@cmliris.harvard.edu
Prof. M. Prato, Dr. S. Campidelli

Department of Pharmaceutical Sciences, University of Trieste

Piazzale Europa 1, 34127 Trieste (Italy)

E-mail: prato@units.it

Prof. R. V. Bellamkonda, I. P. Clements

Department of Biomedical Engineering

Georgia Institute of Technology, Atlanta, GA (USA)

E-mail: ravi@bme.gatech.edu

Prof. G. A. Silva, Dr. S. Pathak

Departments of Bioengineering, Ophthalmology, and Neurosciences Program University of California, San Diego, CA (USA)

E-mail: gsilva@ucsd.edu

Dr. A. Mazzatenta, Prof. L. Ballerini

Department of Physiology and Pathology, Center for Neuroscience B.R.A.I.N. University of Trieste, via Fleming 22, 34127 Trieste (Italy) 
issues motivated us to undertake this work in order to highlight the large number of successes in this field, as well as to address critical problems and challenges that face the field as it continues to move forward.

Until recently the development of neural prosthetic devices for recording and stimulation was primarily fueled by advances in microscale processing of silicon and other electronics-related materials. There were a number of excellent review articles focused on microscale technologies applied to neural electrodes (NEs). ${ }^{[5-11]}$ In this work, the focus is nanotechnology and nanomaterials for the same applications. Nanostructures represent a significant and largely untapped resource for the diagnosis and treatment of injuries and diseases related to neural tissues. From the point of view of a cell biologist, the complex electrical properties and nanoscale structural features of neural tissue necessitate a neural interface with nanoscale components. Nanoengineered materials and devices have the potential to interact with biological systems on a molecular scale, offering unprecedented levels of control over physiological activity. Indeed, many elements of cell machinery, such as ionic channels, adhesion proteins, filopodia, signal proteins, interfacial topography of cell membrane, and elements of cytoskeleton all have nanoscale dimensions, and materials engineered to contact them offer to accommodate these features and functions. Seamless integration of implants with the human body as well as solutions to drug delivery problems through neuroprotective barriers require technological precision at the nanoscale. ${ }^{[12-14]}$ The intrinsic properties of nanomaterials enable the engineering of cellular interfaces ${ }^{[12,15-20]}$ and multiple challenges of long-term communication with live cells make it essential if not inevitable that nanomaterials be used to interface with cells.

The nexus of neurons and nanoscale materials can also be examined from the perspective of a materials scientist. The large class of currently available nanomaterials contains many families of nanostructures, including nanoparticles (NPs), nanowires (NWs), multi- and single wall carbon nanotubes (CNTs), polymer coatings, silicon lithographic elements, and nanodevices. All of these nanostructures have the potential to be juxtaposed with living cells, offering great opportunities for cellular interfacing. Significantly, recent applications of nanotechnology in other fields, such as electronics, optics, and structural composites has provided a newfound wealth of information about the electrical, optical, and mechanical properties of nanomaterials. This rise in knowledge and expertise has already started contributing toward a natural transition of nanomaterials applications from electronics to neural interfacing. As discussed below in Section 2.3, NEs require a combination of a disparate variety of properties-a combination that is becoming increasingly difficult to attain with traditional materials. Practical design aspects of NEs also bring about other potential biomedical applications of nanostructures anticipated for neural tissues, such as neural guidance, differentiation, and drug delivery. Optical and magnetic properties of nanostructures open possibilities of using nanocolloids for improved methods of monitoring excitation in neurons. Overall, our primary focus in this review will be on the use of nanomaterials for NEs and devices interfaced with neural tissues. Drug delivery, imaging, and other similar

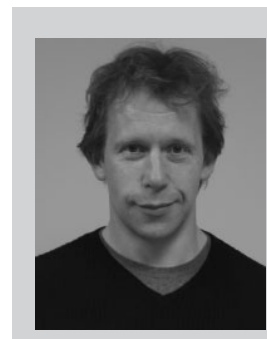

Nicholas A. Kotov earned his B.S. (1987) and Ph.D. (1990) degrees from Moscow State University. After a postdoc at Syracuse University he assumed an Assistant Professor position at Oklahoma State University. At OSU Kotov pioneered the studies of self-organization of nanoparticles and layered composites from nanocolloids. In 2003, he moved to the University of Michigan to become a Professor at the Departments of Chemical Engineering, Materials Science, and Biomedical Engineering. In Michigan, Kotov developed several biological applications of nanoparticles and nanocomposites with strong emphasis on neural interfaces. $\mathrm{He}$ also works on advancing understanding the fundamental similarities between nanoscale and biological systems.

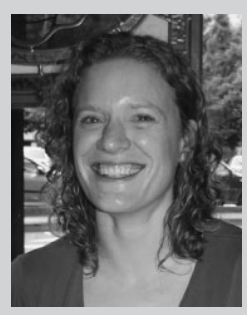

Jessica O. Winter received her Ph.D. in Chemical Engineering from the University of Texas at Austin in 2004. She completed a post-doc fellowship at the Center for Innovative Visual Rehabilitation, a collaborative effort between the Boston VA Hospital, Harvard Medical School, and the Massachusetts Institute of Technology to develop a retinal prosthesis. Since summer 2006 Winter is an Assistant Professor in the departments of Chemical and Biomolecular Engineering (and Biomedical Engineering) at the Ohio State University. Her current research interests include the development of biomimetic materials to improve the electrode/host interface in stimulating prostheses and the development of multi-functional nanoparticles for biological manipulation and imaging.

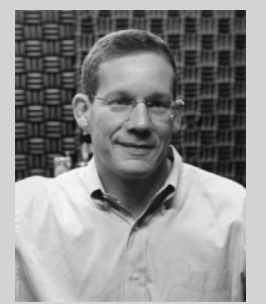

Charles M. Lieber received his Ph.D. in Chemistry at Stanford University followed by postdoctoral research at the California Institute of Technology, he assumed an Assistant Professor position at Columbia University. There Lieber studied the synthesis and properties of lowdimensional materials. He moved to Harvard University in 1991 and now holds a joint appointment in the Department of Chemistry and Chemical Biology, as the Mark Hyman Professor of Chemistry, and the School of Engineering and Applied Sciences. At Harvard, Lieber has pioneered the synthesis of a broad range of nanoscale materials, the characterization of the unique physical properties of these materials and the development of methods of hierarchical assembly of nanoscale wires, together with the demonstration of applications of these materials in nanoelectronics, nanocomputing, biological and chemical sensing, neurobiology, and nanophotonics. Lieber has also developed and applied a new chemically sensitive microscopy for probing organic and biological materials at nanometer and molecular scales. 
systemic functions of nanocolloids will also be discussed, though only in relation to specific tasks related to neural tissues, since much attention to optical and magnetic imaging with nanocolloids has been already given. ${ }^{[21-29]}$

This work comes from a collaborative effort of scientists and engineers from both the nanotechology and neuromedicine sides of the aisle, united by common interests in neural interfacing. The review has three purposes: (i) to review and identify the challenges facing NE development and to establish a framework of materials requirements for such devices; (ii) to comprehensively review recent progress in the application of nanotechnology toward neural interfacing; (iii) to suggest a course for subsequent research and development efforts in this dynamic area.

\section{Current Status of Neural Interface}

\subsection{An Overview of Traditional Neural Electrodes}

The history of biomedical use of neural electrostimulation tissues goes far back in time. To a large degree it accompanies the process of discovery of electricity by mankind. Ancient Egyptians (2500BC) used $400 \mathrm{~V}$ electrical shock from electrical catfish as a pain relief ${ }^{[30]}$ and as a treatment for many ailments including hemorrhoids. There are also speculative pieces of evidence that electrical stimulation was also used in religious ceremonies as a method to affect the spirit of life $\mathrm{ka}^{\left[{ }^{[31]}\right.}$ Ancient Greeks applied electrical impulses from electric eels in foot baths helped pain relief and enhanced blood circulation. In $47 \mathrm{AD}$, a physician Scribonius Largus accidentally came into contact with an electric torpedo fish (Black Torpedo Ray) and suddenly developed a cure for gout, which later was accepted in general practice. ${ }^{[32]}$ The first scientific studies of stimulation of effect of electricity on animals are often incorrectly attributed to Luigi Galvani ${ }^{[33,34]}$ who introduced a fairly vague concept of animal electricity after experiments of electrical stimulation of frog legs published in 1791. ${ }^{[35,36]}$ However, one of the Founding Fathers of the United States of America, who also was a prolific inventor and scientist, Benjamin Franklin, investigated the effect of the muscle contraction that followed electrical shock as early as $1759 .{ }^{[37,38]}$ There are also indications that Peter Abildgaard, the founder of Royal Danish Veterinary College, was also one of the first scientists to demonstrate the contraction of muscles by electrical stimulus. ${ }^{[31]}$

The next generation of NEs did not differ that much from the 1700 s versions by design principle but differed significantly in respect to their physical dimensions. The reduction in size of NEs allowed Hodgkin and Huxley to study electrical signals from individual neuronal fiber in 1939 and conceptually change this field of knowledge: it led to extensive development of signal transduction theory in neurons. ${ }^{[39]}$ Not surprisingly, the next pivotal step in NE development began with the new level of dimensional control over these devices. Development of micromachining resulted in introduction of a single metal wire electrodes and early wire array electrodes, ${ }^{[40,41]}$ which eventually led to the revolution in NE technology and transition from the laboratory to large number of patients. As such, after being approved by the Food and Drug Administration (FDA) in 1985 for adults and in 1990 for children, 70000 cochlear prostheses were implanted worldwide by $1999 .{ }^{[42]}$ According to the FDA data from 2005, nearly 100000 people worldwide have received cochlear implants. In the end of 1960s and early 1970s, the research efforts at Stanford University and the University of Michigan by Angell, Starr, Wise, and later Najafi resulted in the first silicon-based NEs manufactured by using lithographic and etching technologies. ${ }^{[43,44]}$ Later in the $1990 \mathrm{~s}$, Normann and co-workers ${ }^{[45]}$ from the University of Utah applied methods of microscale sawing and columnar etching to produce one-chip arrays of NEs for long-term intracortical implantation. Considering this historical timeline, one can project the next conceptual step in the development of NEs will be associated with nanoscale processing of materials.

Nowadays, diagnostic, therapeutic, and treatment strategies for neural problems rely increasingly on electrical stimulation and recording techniques. Besides cohlear implants, deep brain stimulation (DBS) ${ }^{[46-49]}$ is probably another good example of the rapidly growing field of medical practice for NEs. Also, central nervous system (CNS) implants has been developed to correct motor disorders, ${ }^{[50]}$ while implants in peripheral nervous system are used or control pain, muscle contraction, and activity of specific organs. CNS implants are also considered as a device to translate willful brain processes into actions of external electronic or mechanical systems. ${ }^{[51-53]}$ Notwithstanding a certain degree of controversy regarding their efficacy, safety, and ethical appropriateness underscoring the need for further studies and device improvements, NEs have significantly improved the quality of life for an increasing number of people suffering from neurological disorders and injuries. ${ }^{[54,55 a]}$

Since their discovery, penetrating electrodes (Fig. 1A-C and G) and cuff electrodes (Fig. 1D-F) have been conceptualized and reduced to practice to meet more specific needs. The penetrating electrodes, such as the so-called Michigan electrode (Fig. $1 \mathrm{~A}-\mathrm{C})^{[10]}$ or Utah electrode (Fig. $1 \mathrm{G}$ and $\mathrm{H}$ ), ${ }^{[34]}$ are used for stimulation and recording of brain tissues, while the cuff electrodes are implanted around peripheral nerves. Both penetrating and cuff NEs (Fig. 1A and F) may have several points of electrical interface with cells, allowing for the simultaneous stimulation or recording of multiple areas. NEs are sometimes considered as prototypes of brain-machine interface. In general, the direct connection between brain and a computer is certainly an interesting and motivating goal, which is worth considering. ${ }^{[56]}$ As a fairly remote possibility, such bio-electronic devices may remove the information junction point represented by the keyboard used to type this sentence. As a closer target, direct information exchange technologies can greatly help paralyzed individuals and amputees. ${ }^{[52]}$ The use of nanostructured materials toward these technologies is expected to be most essential and, most likely, inevitable. However, we would like to emphasize that hyperbolization of this subject by some scientists and science press overshadows many important health problems that can be alleviated by the use of NEs as well as technical issues.

Aside from establishing an electronic communication link with neural tissue, there are other immediate and acute needs for developing NEs related to devastating diseases. For example, chronic deep-brain stimulation with implanted electrodes has 

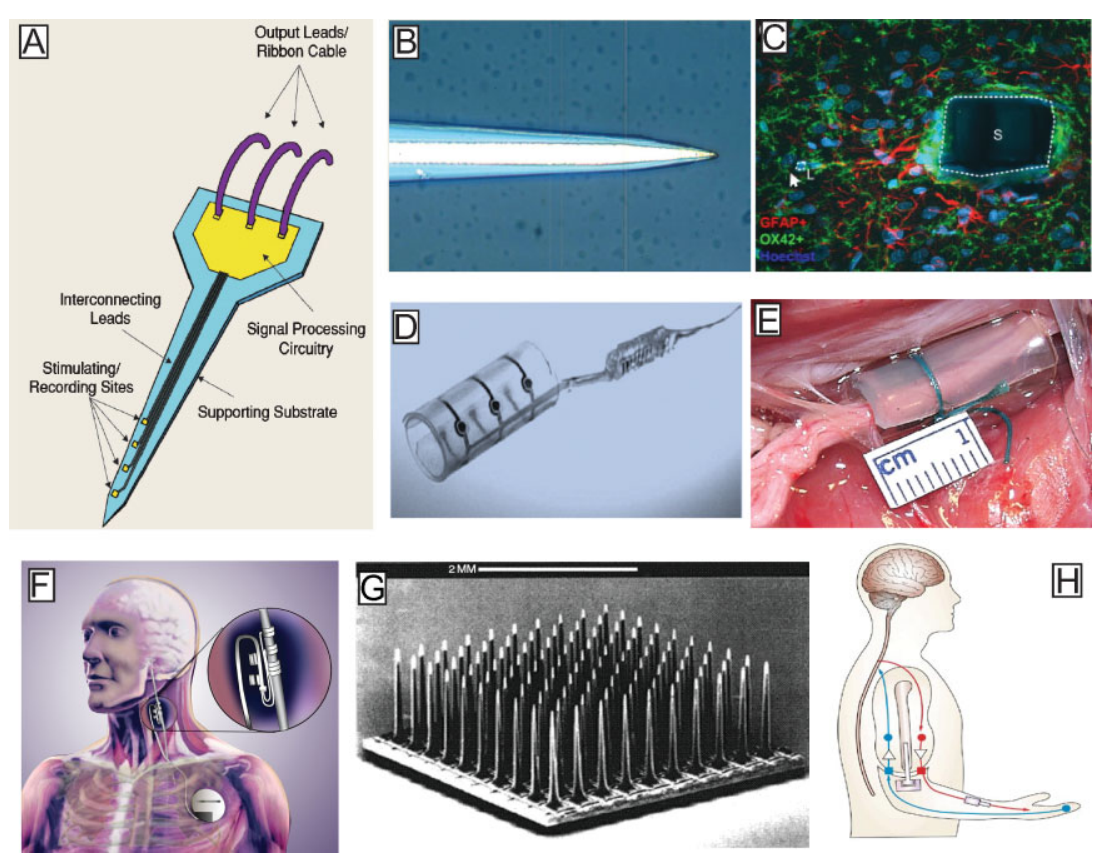

Figure 1. A) Brain stimulation electrode developed in Michigan, the so-called "Michigan electrode"; B) close-up of the implantable part in earlier models. The single recording site is at the tip of the shank. Both images were reproduced with permission from [10]. Copyright 2005, IEEE. C) Encapsulation of implanted model of Michigan electrode marked as $\mathrm{S}$ in the brain of a rat: red stain is glial fibrillary acidic protein typical for astrocytes (GFAP), green stain OX-42 protein typical for microglia and macrophages, blue stain Hoechst 33342 dye with cell nuclei specificity. Much smaller model marked as $L$ has much lower concentration of astrocytes and microglia around it. Reproduced with permission from [111]. Copyright 2007, Elsevier. D) Typical cuff electrode. Reproduced with permission from [55b]. Copyright 2005, Blackwell. E) implanted cuff electrode over sciatic nerve of a cat. Reproduced with permission from [112]. Copyright 2006, IOP. F) schematics of the typical implantation of a cuff electrode around the vagus nerve (picture is adapted from an original attributed to Cyberonics, Inc.); G) array electrode developed in the University of Utah, the so-called "Utah electrodes"; H) schematics of the potential next generation prosthetic arm driven by recording and stimulation from Utah electrodes (both from [34]). Both images were reproduced with permission from [34]. Copyright 2007, Nature Publishing Group.

proven to dramatically alleviate symptoms in patients of Parkinson's ${ }^{[50,57-59]}$ and even Alzheimer's diseases. ${ }^{[60]}$ NEs have also allowed patients of amyotrophic lateral sclerosis and paralysis to regain their motor control and function. ${ }^{[9]}$ Cuff electrodes around peripheral nerves are used for treatment of numerous disorders from loss of hearing, chronic pain and Parkinson's disease to depression and arrhythmia. ${ }^{[61-71]}$ NEs also offer the potential for restoring mobility of limbs and other parts of the body in case of injury or paralysis (Fig. 1H). ${ }^{[72]}$

Despite general success and proven clinical relevance, for instance with DBS and cochlear implants, there exist practical and fundamental challenges related to the utilization of NEs. While most chronically implanted electrodes appear to be well tolerated initially, long-term electrode viability remains a significant problem. Reduction of electrode size without sacrificing the electrode's ability to transfer charge to a cell is critical for improving stimulation techniques. From the biological side, much is still unknown regarding the response of neural tissue to the long-term presence of implanted electrodes. Significant strides toward more effective, smaller, and safer electrodes hinge on improvements of biocompatibility, charge transport, mechanical compliance with the surrounding tissue, chemical stability of the electrode materials, the ability to influence neuronal growth, and drug delivery capabilities. ${ }^{[46,47,49,72-75]}$ Technologies geared toward elucidating the interaction pathways of neuronal networks are also in the early stages of development and will require new methods of imaging and signal tracing.

\subsection{Brief Overview of Electrical Processes During Electrostimulation and Recording}

In order to better appreciate the problems and to evaluate nanostructured materials from the perspective of NEs, it is necessary to give a brief overview of the actual electrical processes taking place at the neuron/electrode interface. The ability to interface with neural tissue is anchored in the movement of electrical charge between the electrode and the surrounding tissue. ${ }^{[47,49,72,73,76]}$ We need to point out that we will give here fairly simple description of the processes, which is sufficient to appreciate the key challenges of the field related to design of new materials but does not present the process of neural stimulation in all complexity of the events taking place. We omitted the detailed information on extracellular current flows, the mechanisms of neuron reactions to an electric field or an injected current, sequence of closing and opening of ion channels, current and novel methods of recording of neural potential including subthreshold signals, and only briefly discussed the reaction of tissue as a whole to electrical stimulation. It was done because there are excellent reviews written on these subject and we encourage the reader to supplement the information provided here with additional details when they are required. ${ }^{[77-81]}$

The electrical processes taking place during stimulation and recording are fairly identical, with the exception that the sequence of charge movements is reversed and magnitude of currents in the recording modality is smaller. For stimulation, charges are delivered to the electrode and induce the shift in the membrane potential of the neuron. For recording, the fluctuations of membrane potential cause the current to go in and out from the electrode. Having an effective interface is a prerequisite for both stimulation and recording although the amount of charge required for stimulation is orders of magnitude higher than what is recorded. The optimal dimensions of the electrode which are inherently tied to its electrical properties can also be quite different for stimulating and recording.

For safe stimulation, the electrode must deliver appropriate charge to the interface and tissue without inducing chemical 
reactions on the electrodes. Faradaic processes resulting from reduction-oxidation (redox) electrochemical reaction between species in solution and electrodes generally must be avoided during cell stimulation except in the special case of some rare materials such as Ir (see below) ${ }^{[82-85]}$ Maximization of the capacitive or non-Faradaic charge storage arising from movement of charged ions in the electrolyte toward or away from the electrode is a critical consideration in successful NE design. ${ }^{[47,49]}$ Large electrode surface area accessible to ions can greatly increase the capacitive component. The material selection for the electrode is, thus, a critical determinant for this process as the materials' structure can significantly impact the surface area.

Electrical stimulation is typically carried out by a biphasic rectangular signal with equal cathodic and anodic charge distribution (net charge $=0$ ). The charge per phase $\left(\mathrm{in} \mathrm{mC} \mathrm{ph}^{-1}\right.$ or $\mu \mathrm{Cph}^{-1}$ ) and charge density (in $\mathrm{mC} \mathrm{cm}^{-2}$ ) are derived from the integral over one phase of a waveform. ${ }^{[7]}$ These two parameters are commonly employed to determine the threshold conditions for triggering injury during electrical stimulation, thus stressing the importance of the stimulation current magnitude and frequency, as well as the electrode size, for safe and efficient stimulation. The charge injection limit is determined by the amount of charge that can be injected without causing electrode degradation or electrolysis of water. The size requirement for electrodes typically requires the electrode to be small enough to enable selective stimulation of a targeted population of neurons, with an area typically $\leq 2000 \mu \mathrm{m}^{2}{ }^{[86]}$ Several materials have already been identified as biocompatible, corrosion-resistive candidates for electrical stimulation and implantation, including noble metals such as $\mathrm{Au}, \mathrm{Ti}, \mathrm{TiN}, \mathrm{Pt}, \mathrm{Rh}, \mathrm{IrO}_{x}$, stainless steel, alloys of these metals, highly doped semiconductors such as silicon, and conducting polymers. Two electrode materials are used most commonly in practical medicine: $\mathrm{Pt}$ and iridium oxide $\left(\mathrm{IrO}_{x}\right)$. Pt is resistant to corrosion and has charge storage capacities in the range of $300-350 \mu \mathrm{C} \mathrm{cm}^{-2}$. Platinum/iridium $(\mathrm{Pt} / \mathrm{Ir})$ alloys have been explored to boost the mechanical properties of the electrode without sacrificing its charge capabilities. In the case of iridium oxide $\left(\mathrm{IrO}_{x}\right)$, reversible redox reactions

$\mathrm{Ir}^{2+} \rightarrow \mathrm{Ir}^{4+}+2 e \quad$ and $\quad \mathrm{Ir}^{2+} \rightarrow \mathrm{Ir}^{3+}+1 e$

occur at the electrode/media interface and significantly increase the charge storage capacity of this material. The product of these reactions, commonly denoted as $\mathrm{IrO}_{x}$, forms a thin film on the top of the electrode in the vicinity of the cells (Fig. 2) and boosts the charge injection to the range of $2-3 \mathrm{mC} \mathrm{cm}^{-2}$. Safe limits for electrical stimulation of tissue vary depending on the target of interest; a typical charge of $0.2-0.5 \mu \mathrm{C}$ is needed.

\subsection{Current Challenges of Neural Electrodes}

\subsubsection{Reduction of Size}

It is desired that NEs selectively excite or record from one neuron at a time or even from one neurite at a time. Reaching these
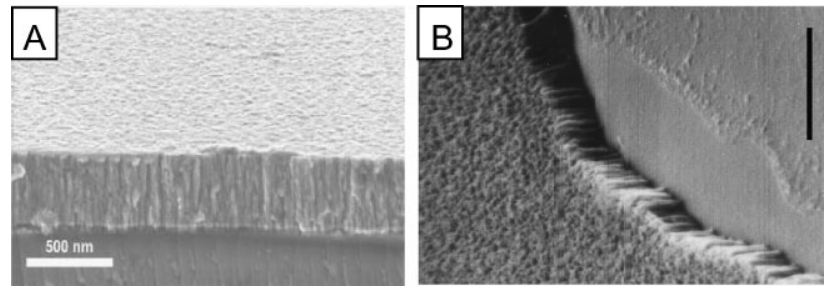

Figure 2. A) SEM image of IrO electrode, scale is $0.5 \mu \mathrm{m}$. Reproduced with permission from [82]. Copyright 2007, ECS. B) SEM image of Au electrode with a layer of impedance-reducing porous TiN film, scale $0.75 \mu \mathrm{m}$. Adapted with permission from [33]. Copyright 2002, Annual Reviews.

capabilities will make possible not only fine-tuning of the curative effect of established medical treatments with NE but also fundamentally novel neuroprosthetic devices. Reduction of the size of the electrode is also intrinsically related to the reduction of injury to the tissue and scarring, which represents one of the most important problems for NEs (see Section 2.3.3). $\mathrm{Ir} / \mathrm{IrO}{ }_{x}$ allows one to reduce the size of electrodes compared to Pt or $\mathrm{Au}$ electrodes; but, nevertheless, the electrodes currently used in neurology are substantially larger than single cells having excitation/recording areas extending $70-150 \mu \mathrm{m}$ in all directions from the electrode. This volume contains from dozens to hundreds of cells. To a large degree, the difficulties with further miniaturization are related to the fundamental limits of currently used materials in respect to charge storage capacity and impedance. Further reduction of electrode size requires not only the development of new materials but also different methods of excitation and recording using field effect transistors (FETs, see Sections 5.6 and 5.7), for example, rather than simple metallic spikes. In the case of FETs, however, the gap between the neuron and the electrode has much greater effect on the electrode performance than in traditional implants, such as Michigan electrodes, thus requiring much better adherence of the surrounding cells to the surface of the device. In turn this requirement becomes more difficult to accomplish as the size of electrode diminishes.

\subsubsection{Problems Related to Mechanical Properties}

Mechanical properties of NEs have been in the center of their development for a long time. They were considered however primarily from the stand point of having high enough strength and stiffness to penetrate through pia arachnoid memberane on the brain tissue ${ }^{[87]}$ and a drawback of some plastic electrodes was considered to be difficulty with their insertion, so that the implant site needs to be made by cutting the tissue. ${ }^{[88]}$ Here we will consider the mechanical properties primarily from the standpoint of long-term biocompatibility.

Many common NEs except some electrodes for DBS, Utah electrodes, and some others are made by the deposition of a conductive and high charge capacity metal layer onto a lower performance substrate (Fig. 1). Even the formation of an $\mathrm{IrO}_{x}$ layer on metal electrode by electrochemical means or deposition of an impedance-reducing TiN layer on Au by vacuum sputtering results in a sharp interface between the materials (Fig. 2) which generates mechanical stress between them, which is relieved only 
when one material becomes separated from the other. A simple demonstration of the great importance of mechanical properties for NEs is the fact that thin metal coatings can withstand only limited number of flexural motions without cracking of the lower performance substrates. This limitation is particularly significant in the design of cuff electrodes. For currently used spike-like NEs, strength and stiffness of the electrode shaft is essential for implantation as well as for successful integration with tissue. Mechanical properties of the material in contact with the cells also have tremendous effect on their biological behavior. ${ }^{[89,90]}$ These examples highlight the need to focus attention on mechanical properties of NEs, such as strength, stiffness, toughness, and adhesion.

Delamination of the conductive interfacial layer has been reported in many types of metal electrodes. Delamination results in the deposition of inflammatory tissues and proteins onto the electrode ${ }^{[86,91]}$ but even more damaging is the formation of a microscale gap between the device and the surrounding neural tissue. This gap causes significant reduction in the recording and charge injection capabilities over time because a layer of electrolytes slips between the conductive coating and the backing of NE, causing multiple problems for electrical stimulation of the cells. ${ }^{\left[{ }^{[2]}\right.}$ Eventually delamination leads to complete failure of the electrode.

\subsubsection{Inflammatory Response of Neural Tissues}

The goal of every implantation is to achieve a stable interface, as characterized by minimal cellular change in the surrounding tissue and sustained functioning of the implanted device. However, the functionality of currently used NEs is limited due to the biological response that results from electrode implantation and their constant presence in the host tissue. ${ }^{\text {[3-95] }}$ This chronic inflammation takes the form of glial scarring (Fig. 1C) which results in replacement of neuronal cells with glial cells, ${ }^{[96,97]}$ loss of electrode function due to encapsulation, ${ }^{[93]}$ and impediment to axonal re-growth. ${ }^{[98]}$ Implantation of NEs usually results in localized activation of resident immune cells, ${ }^{[99]}$ such as reactive astrocytes, taking shape as both an early and a sustained response occurring over a long period of time. ${ }^{[94]}$ In general, microglia and astrocytes respond in short times after implantation. Immunohistochemical markers specific for these cells are up-regulated during the first few days and may remain elevated for months, but may subside over time depending on the type and severity of the perturbation. ${ }^{[99-101]}$

Regarding the long-term (3-6 months) inflammatory response, various studies have reported astrocytic elements to surround and grow on (i.e., encapsulate) electrodes implanted in the CNS. This encapsulation causes an eventual increase in NE impedance, even when systemic treatment with anti-inflammatory agents was used. ${ }^{[93,102,103]}$ Significantly, the encapsulation response seems to be universal, occurring following implantation of a variety of materials, including various metals, semiconductors, and polymers. ${ }^{[94]}$ It was hypothesized based on strong supporting evidence that the failure of the NE devices over the long term is associated in part with micromotions of the electrode in relation to the surrounding neural tissue. ${ }^{[104-107]}$ Micromotions originate with natural movements of the head as well as with variations of blood pressure. The resulting mechanical stresses on the cells at the tissue electrode interface stimulate reactive astrocytes, which perform immune functions in the CNS. ${ }^{[104,108-111]}$ The large mismatch between the mechanical properties of tissues and electrodes is the main contributor of mechanical stress and micromotions. ${ }^{[104,112]}$ Damage to the neurovasculature caused during implantation is also believed to contribute to the inflammatory response $\mathrm{e}^{[113,114]} \mathrm{due}$, in part, to infiltrating immune cells and other non-local cellular elements such as cytokines. ${ }^{[115,116]}$

The second approach to inflammation primarily considers physical dimensions of electrodes implanted in neural tissues. The size of implanted objects seems to make considerable difference in respect to activation of immune system and inflammation (Fig. 1C). Macrophages in cell cultures do not adhere to or spread on polymer fibers ranging in diameter from 2 to $12 \mu \mathrm{m} .{ }^{[117]}$ Decreased capsular thickness was observed when polymer fibers with a diameter from 2.1 to $5.9 \mu \mathrm{m}$ were implanted in the subcutis. ${ }^{[18]}$ Physical dimensions of surface features were found to affect mechanotrunsduction, adhesion, and apoptosis. ${ }^{[102,119,120]}$ The direct studies of the electrode size on encapsulation also strongly suggest that inflammation response is substantially reduced as the NEs become smaller (Fig. 1C). ${ }^{[95,112]}$ Technologically, manufacturing of smaller electrodes is possible; however, the materials used for making electrical pads on them (see Fig. 1B) do not provide sufficient charge injection capacity, interface impedance, or actual conductivity for successful reduction of NE dimensions. We regard this approach as a complementary one to micromotions, and eventually one would need a soft, highly conductive electrode of small dimensions to mitigate encapsulation.

\subsubsection{Necessity to Guide Neurons}

Inflammation processes around NEs can lead to the replacement of neurons in the vicinity of NEs with glial cells as described in the previous section. Following the formation of the glial scar, the number of neurons around the implanted electrode is greatly reduced. Consequently, it would be desirable to guide the neurons or their processes back to within a close vicinity of the NE surface. Peripheral nerve interfaces designed to interface with regenerating axons from an amputated nerve stump are another example of a neural interfacing device that would benefit from the development of materials capable of guiding neuronal growth. ${ }^{[121]}$

The same challenges are also present in the field of peripheral nerve repair. Long nerve gaps are currently bridged with autografted segments of nerve, but this treatment has several major drawbacks, including the need for a secondary surgery and loss of donor site function. A potential alternative is to bridge nerve gaps with guidance scaffolds containing materials able to stimulate and direct axonal regeneration toward the distal stump (see Section 4.1.3).

\subsubsection{Poor Understanding of the Action Mechanism}

There is a significant challenge to understand the mechanism of NE functionalities for DBS and cuff electrodes. The body of knowledge about the propagation of the signal in nerves during the cuff electrode simulation contains many blank spots, but this situation is particularly peculiar for DBS. While being clinically 
effective for the treatment of Parkinson's disease, traumatic brain injuries, paralysis, multiple sclerosis, depression, obsessivecompulsive disorder and other malfunctions, DBS treatment was found empirically. Only in single cases have fairy detailed studies of DBS mechanisms were carried out. As such, reduction of tremors in Parkinson's disease is associated with the enhanced release of adenosine. ${ }^{[122]}$ However, in general, essential mechanisms of DBS are unknown. ${ }^{[122-125]}$ A number of plausible hypotheses explaining the clinical outcomes of DBS have been offered. However, they are based on either inhibition or stimulation of activity in the target brain structure. ${ }^{[126]}$ In the first case, neural output is regulated indirectly by the activation of axon terminals that make synaptic connections with neurons near the stimulating electrode. In the second case, synaptic inhibition $^{[127]}$ and depolarization blockade $\mathrm{e}^{[128]}$ may occur when stimulation by NE alters the activation of voltage-gated currents, which block neural output. For instance, reduction of activity of neurons in orbitofrontal cortex was associated with the success in treatment of obsessive-compulsive symptoms. ${ }^{[129]}$

This lack of understanding in how neuronal networks interact with NE brings about the critical need of developing new model systems for the study of signal propagation and associated cellular processes taking place between neurons. Improvement of imaging of different states of nervous tissue using both optical and magnetic means will also be of great value here. These are being actively pursued by many scientists working in nanotechnology. High contrast agents sensitive to the membrane potential of neurons with short reaction times are much needed as well. Semiconductor and magnetic NPs are being investigated for these purposes. ${ }^{[130-136]}$

\section{Biocompatibility of Nanomaterials with Neural Tissues}

Applications of nanomaterials to the problems of the neural interface must start with discussion of the potential concern of biologists, physiologists, neurologists, and other scientists about biocompatibility of nanostructures and neural tissue. Investigations of toxicity are partially instigated by the substantially different properties that nanostructures exhibit relative to similar bulk materials. For example, nanocolloids have a considerably higher surface to volume ratio than bulk materials. It is precisely this attribute which confers many of the positive features linked to nanostructures being used for NE or drug delivery tasks, but this attribute also significantly alters the biological pathways in which nanostructures can participate and the kinetics of those interactions. ${ }^{[137]}$

A good example of this phenomenon is the ability of nanostructures to translocate across the brain-blood barrier (BBB) through the olfactory bulb. ${ }^{[138]}$ This pathway provides an entry point to the CNS that seems unique to objects in the nanosize regime. Once in the CNS, nanostructures can migrate to additional parts of the brain, traveling along axons and dendrites and even crossing synapses. ${ }^{[137]}$ In the case of neural prosthetic interfaces, nanostructures would originate in the nervous system as a component of a device or be intentionally introduced there; however, it is likely that they could use many of the same mechanisms to travel throughout the CNS. Thus, to fully understand potential nanostructure toxicity, it will be important to examine the long-term stability of materials of NEs and/or other nanoscale systems, such as NPs, NWs, single wall carbon nanotubes (SWNTs), etc. that can be used for imaging purposes in vivo, and to determine their ultimate distribution within the CNS. These questions are not easily answered, and are dependent on not only on the type of nanocolloids employed, but also their surface modification, synthesis route, and size.

In this review, we shall focus mainly on the biocompatibility of nanocolloids such as NPs and CNTs as the most controversial ones. The other nanostructures made, for instance, from silicon or some biological polymers present much less contention. Both $\mathrm{NPs}^{[139-142]}$ and $\mathrm{CNTS}^{[143-150]}$ have shown toxic effects in general cell culture when dispersed in liquids or gas. In depth study has demonstrated that toxicity is linked to specific formulations or material presentations. For example, SWNTs in solid compositions or coatings appear to be exceptionally well tolerated by neuronal tissues. ${ }^{[19,151-154]}$ Creating non-toxic interfaces with neural tissue will require a thorough understanding of these relationships as well as development of new tools for the assessment of neurotoxicity of the components.

\subsection{Toxicity of Nanoparticles}

Optical properties of semiconductor NPs also known as quantum dots open very interesting perspectives for new spectroscopic tools to observe changes in membrane potential of neurons. This is one of the key tasks in improvement of the understanding of neural processes because the traditional voltage sensing dyes based on redistribution of charged molecules between phases, $\mathrm{pH}$ transitions, and spectral shifts in electrical fields used now are either too slow or provide insufficient signalto-noise ratio (or both). Development of a new generation of voltage sensitive membrane-resident labels is much needed, and NPs can theoretically provide this opportunity due to high polarizability of excitons. However, the problems related to their cytotoxicity need to be taken into account.

There are numerous studies about cytotoxicity of NPs from different materials and to different types of cells. ${ }^{[155-163]}$ Overall, the most predominant although not the only paradigm of NP cytotoxicity is that they stimulate generation of reactive oxygen species (ROS) and interfere with function of mitochondria. Here we will focus mainly on interactions of NPs related to nervous system and on semiconductor nanocolloids. Metal and metal oxide NPs, such as $\mathrm{Au}, \mathrm{Ag}, \mathrm{Fe}_{3} \mathrm{O}_{4}, \mathrm{Fe}_{2} \mathrm{O}_{3}$ (see Section 4.2), and $\mathrm{SiO}_{2}$, are generally much less toxic and controversial. Moreover, some of them are already used in clinical practice. Toxicity of organic NPs (see Section 4.1), such as liposomes, solid lipid NPs, and nanocolloids from biodegradable polymers has been discussed in literature extensively and should be reviewed here. ${ }^{[164-169]}$

Several studies report toxicity of semiconductor NPs, such as $\mathrm{CdS}$, CdSe, or CdTe, to neural cells, with effects including changes in cell morphology, ${ }^{[170]}$ depressed metabolic activity, ${ }^{[170]}$ decreases in cell viability, ${ }^{[171]}$ and increased lipid peroxidation. ${ }^{[172]}$ For example, PC12 neuroprogenitor cells exposed to cysteamine terminated CdTe NPs $\left(>10 \mu \mathrm{g} \mathrm{mL}^{-1}\right)$ exhibited changes in cell 
morphology after only $1 \mathrm{~h}$ of exposure. ${ }^{[10]}$ Similarly, IMR-32 neuroblastoma cells experienced a decline in cell viability upon short-term exposure to CdSe NPs. ${ }^{[171]}$ However, neurons are not the only, or even the most commonly, affected cell type in the CNS.

Given that astrocytes have a key role in the brain's response to stress, they may provide the best indication of nanotoxicity in the neural system. Astrocytes have two general phenotypes a resting/ quiescent type and a reactive type, in which a specific protein GFAP is expressed in the cell membrane. Such cells are denoted as GFAP+ astrocytes and are typically produced in response to injury or a foreign body. Activation to this reactive form is a strong indication of a toxic response. In a recent study, it was found that NP uptake was enhanced in astrocytes relative to neurons, and that GFAP expression was upregulated, although the length and intensity was dependent on the type of NP investigated. ${ }^{[173]}$ In addition, another immune-like cell type, microglia, and in particular those that act as brain phagocytes, were activated by NP injections. ${ }^{[173]}$ These results indicate that NP exposure can induce an immune response in the CNS.

However, despite their clear potential for toxicity, several reports describe the use of NPs with no ill effects. For example, no difference in ion channel conduction for cells exposed to CdSe/ ZnS NPs in the nM regime was found. ${ }^{[139]}$ A different study did not reveal any evidence of toxicity in commercially available CdSe NPs conjugated to nerve growth factor (NGF). ${ }^{[14]}$ NGF-NPs activated the expected tyrosine kinase A receptor, inducing neurite outgrowth and extension; albeit at a somewhat lower rate than free NGF. ${ }^{[174]}$ These results underscore the fact that NP toxicity is a complicated and controversial issue, with toxicity being profoundly influenced by composition (core and ligands), size, and localization.

\subsubsection{Toxicity Attributed to Nanoparticle Composition}

NPs are composed of materials (e.g., cadmium, lead, mercury, sulfur, selenium, or tellurium) that can be toxic individually. Notably, cadmium, a primary constituent of the most common type of particles employed (e.g., CdSe), is a carcinogen, and has been linked with cancers of the lung, prostate, and kidney. ${ }^{[175]}$ Carcinogenic effects are believed to result from interference with DNA repair processes, ${ }^{[176]}$ possibly by cadmium binding to repair proteins. Cadmium also binds to metallothionein protein, disrupting zinc metabolic pathways in the liver and kidney. More specific to the CNS, cadmium is a known neurotoxin, influencing lipid peroxidation. ${ }^{[177]}$ Cadmium persists in biological systems, meaning that toxic effects can occur long after the initial exposure. However, the effects described here are for free cadmium. In NP form, cadmium ions are complexed with other materials, which may render them less toxic.

Similarly to other NPs, the most likely cause of quantum dot toxicity is the formation of ROS resulting in the so-called oxidative stress. ROS diminish cellular respiration, increase permeation of the cell membrane, and damage the nucleus, leading to eventual cell death. ${ }^{[141]}$ For cells exposed to NPs, this loss of cell viability can be attributed to apoptosis, and not necrosis, a finding supported by observed upregulation of apoptosis-associated proteins (e.g., selected caspases, JNK, Bax/Bcl-2). ${ }^{[171]}$ In addition to immediate effects on cell viability, NPs can cause DNA fragmentation ${ }^{[171]}$ and epigenetic changes. ${ }^{[178]}$ In a detailed gene analysis study, it was determined that gene expression was relatively unaltered in the presence of NPs coated with silane and poly(ethylene glycol) (PEG): $\sim 0.2 \%$ of genes tested displayed differences. ${ }^{[179]}$ However, the few genes that did display differences included some involved in cellular stress responses. Further, it was shown that NP exposure can produce epigenetic changes, activating the $\mathrm{p} 53$ pathway. ${ }^{[178]}$ Most initial studies have been confined to evaluation of cell death, which while important, may not reflect subtle and equally damaging consequences that occur at lower, sub-lethal exposure levels. Only recently have researchers employed techniques such as high throughput screening to systematically identify these lower level effects. ${ }^{[179,180]}$

\subsubsection{Toxicity Attributed to Nanoparticle Core}

It is widely believed that the primary culprits in production of ROS are $\mathrm{Cd}^{2+}$ ions, most likely released as a result of photooxidation of the particle core. ${ }^{[142]}$ These ions are thought to bind mitochondrial proteins, diminishing cellular respiration and ultimately leading to cell death. ${ }^{[142]}$ However, $\mathrm{Cd}^{2+}$ ions may not be directly responsible for ROS-induced damage. One observation that supports this theory is that cell viability does not directly correlate with intracellular (IC) $\left[\mathrm{Cd}^{2+}\right]$, as one would expect if $\mathrm{Cd}^{2+}$ ions are the sole cause of cytotoxic behavior. ${ }^{[81]}$ Additionally, when free radical scavenging chemicals (i.e., $N$-acetyl cysteine and vitamin E derivatives $\alpha$-tocopherol or Trolox) are added to cell culture medium, toxicity is reduced for some, but not all, of the compounds tested. ${ }^{[170,171]}$ It is instead possible that toxicity results from ROS that are produced indirectly, say by charge transfer to an intermediate molecule, ${ }^{[181]}$ a property which has allowed NPs to be used in photodynamic therapy. ${ }^{[182]}$ Regardless, it is clear that access to the particle core, is a primary initiator of ROS events.

To minimize access to the core, it is possible to surround the particle in a protective material. For example, layer-by-layer assembled films of CdTe coated with collagen provide some but not complete protection. ${ }^{[183]}$ Marked reduction of the toxicity effect was observed even for fairly thin protein coatings, which was attributed to the reduction of the contact of cells with the NP core and exposure of cells to $\mathrm{Cd}^{2+}$. Similar observations were also documented for $\mathrm{ZnS}$ and bovine serum albumin (BSA) coatings, which dramatically improved cell viability. ${ }^{[142]}$ IMR-32 human neuroblastoma cells exposed to CdSe NPs displayed a 50-60\% decrease in cell viability (MTT assay) over cells exposed to ZnS capped NPs. ${ }^{[171]}$ In general, surface coatings that have shown great efficacy in reducing NP toxicity include ZnS caps, ${ }^{[139,142,171,181]}$ BSA, ${ }^{[142,170,184]}$ and the combination of silane/silica and phosphonate. ${ }^{[139,179]}$

\subsubsection{Toxicity Attributed to Nanoparticle Surface Functionalization}

In addition to the particle core, the type of ligand on the NP surface can affect NP toxicity. In fact, Hoshino et al. ${ }^{[184,185]}$ asserts that NP surface ligands may have a greater effect on cytotoxicity than the NPs themselves. Ligands identified as cytotoxic include trioctyl phosphene oxide (TOPO), ${ }^{[184]}$ the classic organic synthesis ligand, mercaptoundecanoic acid, ${ }^{[184,186]}$ and mercaptan acids (e.g., mercaptoacetic acid and mercaptopropionic 
acid). ${ }^{[139]}$ In most cases, cytotoxicity was manifested as decreased cell proliferation or DNA damage. Although ligands undoubtedly contribute to toxicity, it is doubtful that they are the only, or even primary, cause. For example, in a controlled study of CdSe and CdSe/ZnS NPs with similar surface ligands, the unprotected CdSe NPs were much more toxic than the CdSe/ZnS NPs. ${ }^{[139]}$ This difference was attributed to increased access to the NP core. Similarly, in the same study, when compared to molecules, toxicity of $\mathrm{Cd}^{2+}$ ions played a greater role than that of the ligand (i.e., mercaptopropionic acid). ${ }^{[139]}$ Nonetheless, these studies indicate that ligand selection and removal of synthesis contaminants (i.e., TOPO) are extremely important in developing biocompatible particles.

\subsubsection{Toxicity Attributed to Nanoparticle Size}

Composition is just one facet of NP toxicity. Particle size also influences cell behavior, with smaller particles generally found to be more toxic than larger particles. ${ }^{[170,186]}$ Detailed analysis seems to indicate that this size dependence is a direct result of the surface to volume ratio of small particles. ${ }^{[139]}$ As particle size decreases, the surface to volume ratio increases significantly, and more surface $\mathrm{Cd}^{2+}$ atoms are available for reaction and subsequent cell poisoning. Alternatively, it is also possible that smaller particles are processed through alternate transport pathways. For example, in one study, smaller green NPs, which were localized in the nuclear compartment, were much more toxic to neural cells than larger red NPs excluded from that compartment ${ }^{[170]}$ (Fig. 3). It is hypothesized that access to the nucleus, and hence DNA, may accelerate toxic responses. If so, it will be critical to ensure accurate targeting and core protection of particles for drug delivery and gene therapy studies.

An additional source of toxicity dependence on NP size can originate from the dependence of redox potential and semiconductor band gap on their diameter. Considering the possibility that ROS can be generated on the NP surface, redox properties can exhibit an exceptionally strong effect on the amount of free radicals produced. Alternatively, photoexcitation of semiconductor NPs can lead to independent generation of free radicals from adsorbed photons, which is obviously affected by quantum size effect in the semiconductor core. The enhancement of NPs toxicity upon illumination had been experimentally documented. ${ }^{[142]}$


Figure 3. Microglia exposed to A) red CdTe NPs 6-8 nm in diameter and B) green CdTe NPs $3-4 \mathrm{~nm}$ in diameter at $50 \mathrm{~nm}$ after $24 \mathrm{~h}$ of exposure. C) Exposure to green NPs produces greater reductions in cell viability than larger red NPs (64.9\% vs. $87.6 \%)$, which are excluded from the nucleus. (Courtesy of Dr. Dusica Maysinger, McGill University, Montreal, Canada).

\subsection{Toxicity of Carbon Nanotubes}

In general, the response of neural tissues to CNTs has been positive. ${ }^{[154,187-194]}$ The biocompatibility of CNT at the cellular level has been verified by several studies, ${ }^{[195,196]}$ but the widespread adoption of CNT-based technologies for biological and medical applications requires a cautious approach regarding the potential toxicity of CNTs to the environment and human health. Initial studies have revealed mixed results with respect to the toxicity of CNT. ${ }^{[143,144,147,150,197-199]}$ In regards to neural applications, CNTs have been studied as possible substrates for neuronal cell adhesion ${ }^{[200-202]}$ and as thin films to enhance/ support neural excitation. ${ }^{[19,152,203,204]}$ Toxicity has not generally been observed for CNT mats, coatings, composites, etc., which do not produce freely floating nanotubes (NTs) ${ }^{[19,151-154]}$ However, we still need to be mindful about toxicity of the dispersions addressed in this part of the review because of potential decomposition of the CNT composites used to manufacture NE electrodes (see Section 5.6).

As with NPs, CNT toxicity appears to be a function of many factors, including composition, surface functionalization, and size. A clear understanding of these features will be necessary to design biocompatible biomaterials for neural applications. Dispersion of CNTs have been linked to toxicity in the brain, ${ }^{[138]}$ lung, ${ }^{[144,145]}$ and skin. ${ }^{[143]}$ The nervous system, in particular, has long been a concern for carbonaceous nanomaterial toxicity. For example, an early nanomaterial toxicology study identified that $\mathrm{C}_{60}$ fullerenes molecules (see Section 4.1.2), which have structural similarities with CNTs, increase lipid peroxidation in the brain. ${ }^{[138]}$ Although in vivo studies with CNTs have been few, exposure has been associated with aneurysms, particularly in the cerebellum, which may indicate degradation of the blood-brain barrier. $^{[205]}$ In culture, neurons ${ }^{[206]}$ and astrocytes ${ }^{[18]}$ grown on some CNT surfaces display reduced cell viability and adhesion, when compared to standard growth surfaces. However, one needs to be mindful of possible interference from the particles of catalyst and other admixtures which can account for a large part of toxicity observed in these studies. The presence of small amounts of soluble toxic components should also be considered. In some cases, such reduced viability can continue even after removal from the CNT-coated substrate. ${ }^{[206]}$ In this particular study, cells did not evidence the same effects to conditioned medium incubated with CNTs, implying that soluble toxic factors did not account for reduced viability. Taken together these data suggest that CNTs may induce epigenetic changes that continue to exert effect long after CNTs are physically removed. This possibility is especially troubling because CNTs are extremely resistant to biodegradability, ${ }^{[207]}$ and may persist in the body indefinitely.

Apart from viability effects, CNT films have been shown to alter neurite branching, length and density, and the number of growth cones (Fig. 4). ${ }^{[18,202,206]}$ The exact mechanism behind these changes is unclear. CNT surface charge or polarity may exert an influence, ${ }^{[206]}$ possibly by influencing metabolic processes. For example, soluble CNTs can decrease the ability of neurons to transport $\mathrm{Ca}^{2+}$ ions in response to membrane depolarization, ${ }^{[201]}$ which has been linked to extension of fewer but longer neurites with a reduction in neurite branching. CNTs films have also been shown to increase the frequency of synaptic activity and neuronal firing, ${ }^{[152]}$ possibly as a result of their ability to serve as pathways 




Figure 4. A,B) Examples of two different neurites (green) extending from neuron cell bodies align in the direction of CNT orientation (yellow arrow). Scale bar $=20 \mu \mathrm{m}$. (Figure courtesy of Dr. Thomas Webster, Brown University, Providence, RI).

for current transfer between adjacent cells. While this is not directly a toxic response and is potentially advantageous for neural prosthesis development, CNT-induced increases in neuronal activity should be studied in more detail to preclude the unlikely possibility of atypical neuronal responses. It should be noted that neuronal resting potential and membrane time constant, as well as action potential features, widely accepted indicators of cultured neurons health, were not affected by pure CNT substrates. ${ }^{[152,208]}$

\subsubsection{Toxicity Attributed to Carbon Nanotube Composition}

Similar to NPs, CNT toxicity is linked, in some measure, to composition. All CNTs are composed primarily of carbon. However, there are many different types of CNTs, including SWNTs and multiwalled carbon nanotubes (MWNTs), each of which can have different sizes and surface properties. Although they structurally resemble graphite, CNTs have substantially different electrical properties, ${ }^{[209]}$ and therefore may behave differently in the bulk than in the nanophase. ${ }^{[144]}$ It is notable, however, that graphite produces toxic responses, particularly in the lung. ${ }^{[144]}$ The lung is a specific concern for CNTs because they are manufactured through processes by which they may easily enter the air. ${ }^{[207]}$ This is in contrast to NPs, which are synthesized primarily through liquid routes. Most studies of CNT toxicity examine effects on the lung or alveolar cells, with the typical response being the formation of granulomas. ${ }^{[144,145,210]}$ It is important to point out that lung toxicity results at least in part from mechanical blockage, ${ }^{[145]}$ as opposed to inherent toxicity, and therefore much of this data may not readily translate to neural systems.

A likely cause of toxicity, as with NPs, is the formation of ROS. ${ }^{[143,149,211]}$ ROS are probably created by CNT photoexcitation, which can generate free electrons. ${ }^{[137]}$ ROS production appears to be dose dependent, ${ }^{[210]}$ most likely proceeds through a Fenton-type reaction (i.e., catalyzed decomposition of $\mathrm{H}_{2} \mathrm{O}_{2}$ yielding $\mathrm{OH}^{-}$radicals), ${ }^{[143]}$ and may be related to iron catalyst contamination. It is also possible that CNTs interfere with ROS scavenging pathways (e.g., glutathione) rather than directly producing ROS. ${ }^{[210]}$ Observed ROS-related effects include decreased cell viability through both apoptotic ${ }^{[148,159,210,212]}$ and necrotic pathways. ${ }^{[159]}$ Specific mechanisms of response appear to vary by dose. ${ }^{[159]}$ In contrast to NP studies, evidence for mitochondrial effects is conflicting. ${ }^{[210,211]}$ CNTs bind the formazan product produced in the classic MTT assay to assess mitochondrial activity, ${ }^{[211]}$ skewing results from papers that assess fitness using only this mechanism and possibly contributing to the confusion. Other tests have shown that CNT exposure disrupts mitochondrial membrane potential, ${ }^{[211,213]}$ although it is not clear if this results from ROS production or penetration of the mitochondrial membrane. ${ }^{[213]}$ In contrast to NPs, another substantial cause of unmodified CNT toxicity may be their hydrophobicity. Whereas low concentration of modified CNTs have been shown to successfully traverse the cell membrane without cytotoxicity (Fig. 5), unmodified hydrophobic CNTs may insert themselves between the hydrophobic lipids of the cell membrane, ${ }^{[213-216]}$ resulting in disruption. Internalized CNTs can also provide a physical barrier, disrupting cytoskeletal proteins and organelle placement, ${ }^{[211,213]}$ as well as external mass transfer. ${ }^{[217]}$ This action has been linked to up-regulation of apoptotic proteins ${ }^{[148]}$ and reduced expression of many common cell adhesion proteins, ${ }^{[218]}$ which may account for CNT influence on neurite branching. ${ }^{[201]}$ It is believed that these processes are an attempt to encapsulate the CNTs, by surrounding them with detached apoptotic cells. ${ }^{[217]}$ It is possible that CNTs evoke these responses because of their size similarity to viruses, and up-regulation of several proteins associated with anti-viral response have been observed in response to CNT exposure. ${ }^{[159]}$ Hydrophobicity can be altered through an oxidation process (acid treatment and/or plasma etch) to produce CNTs that are $\mathrm{COOH}$-terminated. Unfortunately, some oxidized CNTs have been shown to be more toxic than their untreated counterparts. ${ }^{[199,212,217]}$

Another complicating factor is that CNTs are frequently contaminated by trace amounts of the nickel or iron synthesis catalyst. ${ }^{[198,207,218]}$ These contaminants may be responsible for a substantial portion of ROS production. ${ }^{[211]}$ For example, SWNTs containing $\mathrm{Fe}$ contaminants have been shown to enhance

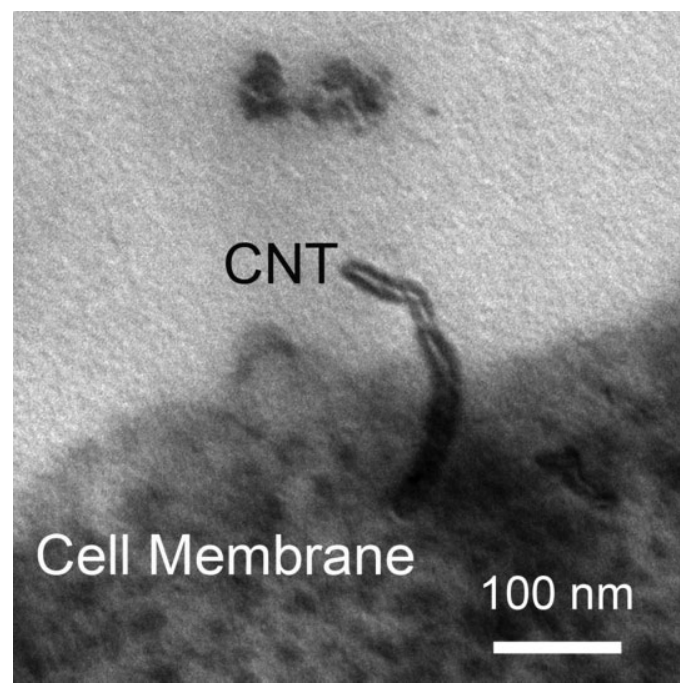

Figure 5. TEM of HeLa cells exposed to ammonium functionalized MWCNTs. CNTs successfully traverse the cell membrane, producing gene transfection. (Courtesy of Prof. M. Prato, University of Trieste, Trieste, Italy; adapted from [216].) 
production of $\mathrm{OH}^{-}$radicals in activated macrophages, which can be converted into $\mathrm{H}_{2} \mathrm{O}_{2}$, inducing oxidative stress. ${ }^{[198]}$ Acid treatment can be used to remove catalyst contamination, and significantly reduces toxic effects ${ }^{[198,217]}$ as compared to the same pristine material.

\subsubsection{Toxicity Attributed to Surface Functionalization of Carbon Nanotubes}

Another influence in CNT toxicity is surface functionalization, both type and degree. Charge, in particular, has been shown to alter neurite extension, density, and length. For example, cells exposed to more positively charged surfaces display more neurite branching ${ }^{[200,202]}$ and growth cones. ${ }^{[200]}$ Polarity also appears to play a role, although to a lesser degree ${ }^{[206]}$ with polar surfaces inducing higher neurite densities than non-polar surfaces ${ }^{[151,203]}$ These results are similar to those for other materials. ${ }^{[219,220]}$ The number of functionalization units also influences toxicity, with increasing units decreasing toxicity. ${ }^{[150]}$ It is hypothesized that improved functionalization protects the NT surface, reducing the likelihood of photooxidation. However, it is also possible that this toxicity results from other factors, including an increased propensity to aggregation (see below).

\subsubsection{Toxicity Attributed to Carbon Nanotube Dimensions}

Size and shape may also play a role in CNT toxicity. From studies in the lung, it has been established that the general chain of toxicity follows the trend SWNTs $>$ MWNTs $>\mathrm{C}_{60}$ (fullerenes) when compared on a mass basis. ${ }^{[221]}$ This result has been difficult to justify based on compositional differences alone, but may be explained by a theory that, in contrast to NPs, materials with the smallest surface area appear to be the most toxic. ${ }^{[218]}$ Other researchers; however, suggest a length dependence in toxicity response. For example, studies examining MWNTs of different lengths (but similar surface areas) found that longer CNTs were more likely to provoke an inflammatory response and were less likely to be engulfed by macrophages. ${ }^{[147]}$ The exact mechanism of these responses is unclear, but it has been suggested that small hydrophobic particles may resemble viruses, inducing an immune response. ${ }^{[159,218]}$ The similarity of CNT size and shape to viruses may influence the toxicity response evoked.

Apart from dimensionality, aggregation state has been shown to have a strong impact on toxicity. Some researchers have found that well dispersed bundles of CNTs are much less toxic than aggregates, which may be as large as micron size. ${ }^{[214,222]}$ Aggregates appear to loosely bind cells, perhaps through their extracellular proteins. ${ }^{[223]}$ These aggregates may influence the ability of nutrients and wastes to traverse the cell membrane. ${ }^{[217]}$ Alternatively, it has been suggested that aggregated CNTs exhibit reduced surface areas that render them less toxic than more well-dispersed materials. ${ }^{[218]}$ However, others have shown that the effect of aggregates is important, but not entirely responsible for toxic effects. ${ }^{[223]}$ For example, when SWNT-containing cell culture medium was filtered to remove large aggregates $(>0.22 \mu \mathrm{m})$, the filtered medium produced toxic responses only slightly lower than that of unfiltered medium. ${ }^{[223]}$ Upon further examination of the filtered medium content, it was determined that the medium predominately contained small SWNT bundles $(<5 \mathrm{~nm})$ and graphite-like particles, which probably compro- mised $1 \%$ of the initial SWNTs by volume. The particles were surrounded by amorphous materials, probably adsorbed proteins, suggesting that one possible mechanism of in vitro toxicity is the removal of serum proteins from culture medium. Because acid and oxidative treatments that are used to remove catalyst impurities can greatly enhance NT aggregation, it will be critical to understand the role of aggregation in CNT toxicity.

\subsection{General Conclusions about Cytotoxicity}

Overall, the set of conclusions that can be reached from the entire Section 3 are the following. (i) The largest component of nanomaterial cytotoxicity is composition. (ii) Apart of some dependence of toxic effects on size and aggregation state, nanomaterials did not demonstrate fundamentally new mechanisms of cytotoxicity which would be specific to nanoscale. This does not mean that they are impossible, but for the materials of interest they are not engaged. (iii) The most significant concerns are raised about semiconductor NPs containing $\mathrm{Cd}^{2+}$. This can be extended to other particles from heavy metals. So, these materials should be avoided for the use in long-term devices, but can still be applied for imaging in animals and ex vivo to establish, for instance, a neuron firing sequence. (iv) Traditional materials used in the design of NE and other implantable devices are not expected to show drastically different mechanisms and/or levels of toxicity after acquiring nanoscale features.

\section{Nanocolloids and Neuronal Tissues}

This part of the review describes the applications of different nanostructures in dispersed state, i.e., nanocolloids, for the purpose of aiding the neural interface. They typically perform the tasks of drug delivery or a contrast agent in imaging, which have immense importance for practical neurology. Drugs can be used to modulate inflammatory response, deliver factors supportive of neural health and neurite outgrowth, and even to introduce genetic material for targeted gene therapy. Temporal and spatial control over the delivery of drugs and biological factors is one of the key aspects of materials design for this function to ensure safety and efficacy of NE and neural tissues in general. Nanoscale particles, structures, and coatings offer the fine control necessary to achieve this degree of selective targeting over controllable timescales.

The same arguments are applicable to the description of advantages of nanocolloids for imaging. Visualization of brain activity and damaged areas of CNS represent continuous challenges despite very significant progress made in recent years. NPs have the ability to penetrate the BBB and accumulate in certain areas of brain. Targeting of contrast agents still needs to be improved greatly.

Besides serving as contrast agents, nanocolloids can theoretically serve as NEs as well. Although it would not be impossible to imagine a self-organized system of NPs or similar species, such as NWs or NTs, that form a conductive electrode-like network in a specific area of the nervous system, the challenges of delivering sufficient power to such a network and lack of a clear delivery mechanism after systemic injection prevents us from discussing 
such options at the present time. It will certainly be of broad scientific interest and importance to keep such systems in mind rather than to dismiss them as an absolute impossibility.

\subsection{Organic Nanocolloids}

In general, controlled delivery of drugs and biological agents can be mediated by many different classes of NPs. In this section, we will consider only those made from organic materials, such as polymer-based NPs, ${ }^{[224]}$ liposomes, ${ }^{[22-227]}$ etc., while inorganic NPs are reviewed in Section 4.2.

\subsubsection{Drug Delivery with Organic Nanoparticles}

NPs have the potential to cross the $\mathrm{BBB}^{[228,229]}$ and can be conjugated with ligands to enable targeting of specific cell types. Liposomes for example, are nanoscale spheres composed of a lipid bilayer surface that can encapsulate or incorporate drugs and biological agents for delivery as exemplified in Figure 6 by liposomes loaded with an iodine CT contrast agent for imaging. Incorporation of polyethylene glycol on liposome surfaces extends their circulation time in the bloodstream, ${ }^{[230]}$ and conjugation of various ligands enables selective targeting of drugs to specific cell types. ${ }^{[225-227]}$

Biodegradable polymer NPs such as poly(lactic-co-glycolic acid) (PLGA) can be used to achieve a tailored release profile of encapsulated drugs over timescales of days up to months. NP mediated drug release can additionally be localized by surface binding of bioactive ligands, ${ }^{[231]}$ incorporation of NPs within hydrogels, ${ }^{[224]}$ and a variety of other techniques. ${ }^{[18]}$ The resulting spatiotemporally controlled release allows for extended modification of neural tissue in the vicinity of implanted devices. As an example, localized delivery of anti-inflammatory agents to the NE-tissue interface has been achieved by coating neural probes with NP containing gels. ${ }^{[224]}$

NP-mediated gene delivery is another application with future potential for influencing the behavior of neural tissue around an

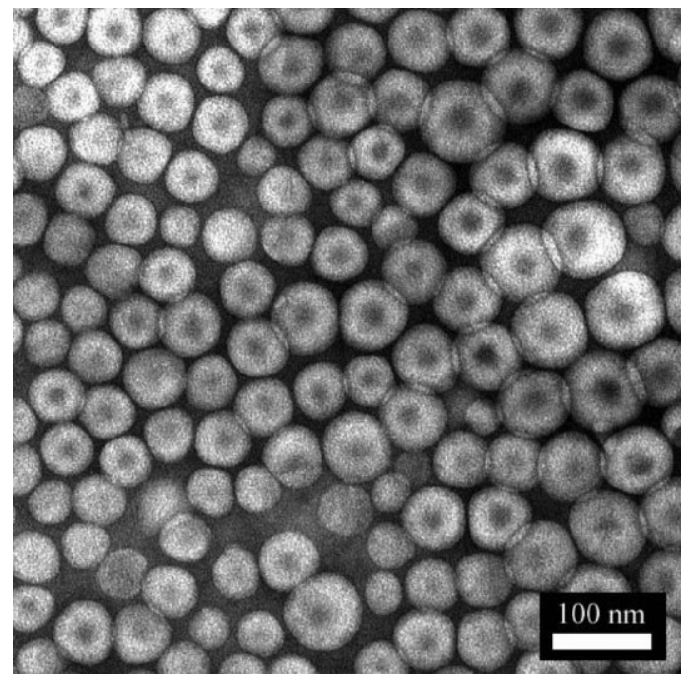

Figure 6. TEM image of loaded liposomes. (Courtesy of the group of R. Bellamkonda, Georgia Institute of Technology, Atlanta, USA). implanted device. Gene therapy offers the potential to modulate neural growth and regeneration, ${ }^{[232]}$ tissue response, ${ }^{[233]}$ and otherwise influence neural tissue behavior in a manner favorable to establishing a neural interface. The use of viral vectors is currently the most efficient and stable technique for genetic transfer into targeted cells. ${ }^{[232,234,235]}$ However, drawbacks including safety concerns and undesired side effects associated with the use of viral vectors have spurred research in the area of NP-mediated gene transfer. ${ }^{[236]}$ The use of liposomes ${ }^{[233,237]}$ or biodegradable polymers ${ }^{[238]}$ conjugated with ligands again offers cell targeting and temporal control of gene delivery.

Polysorbate 80 coated NPs are another example of potential drug carriers across the BBB. These NPs are most likely absorbed via endocytosis by the endothelial cells lining brain capillaries. Polysorbate 80 causes the adsorption of apolipoprotein E from the blood plasma onto the NP surface, creating particles which mimic low density lipoprotein (LDL) particles that interact with LDL receptors, leading to their uptake by endothelial cells. Drug can then be released in the CNS..$^{[13,228,239,240]}$ Imaging studies of these NPs using analytical electron microscopy (AEM) with copper chlorophyll as the contrast agent have confirmed that they do indeed cross the BBB and accumulate in the brain. ${ }^{[241]}$

Another potential use of polymeric NPs is the treatment of Alzheimer's disease by metal chelator-NP complexes. The abnormally high levels of redox-active metals in affected areas of the brain in Alzheimer's patients are difficult to treat with metal chelators because they do not cross the BBB. However, chelator-NP complexes may be a promising approach. They were incubated with human plasma to adsorb apolipoproteins $\mathrm{E}$ and A-I, facilitating transport across the BBB. ${ }^{[242]}$ Cationic albumin conjugated NPs being developed for gene delivery to the CNS have also been shown to cross the BBB quickly and effectively following systemic intravenous administration. ${ }^{[174,243]}$ The cytotoxic products of genes such as the proapoptotic Apo2 ligand/tumor necrosis factor-related apoptosis-inducing ligand (Apo2L/TRAIL) may potentially be used to treat malignant tumors by inducing targeted apoptosis (programmed cell death) of cancerous cells. However, their delivery to the CNS is hampered because conventional viral vectors cannot cross the $\mathrm{BBB}$, in addition to other safety considerations associated with viral gene delivery vectors. If the safety and biocompatibility of cationic albumin conjugated NPs can be established, this system may prove to be a feasible non-viral gene delivery vector for primary CNS tumors. Initial experiments using this system have induced apoptosis in vivo and delayed tumor growth. ${ }^{[244]}$

\subsubsection{Fullerene Derivatives}

Fullerenes are being investigated as drugs and for small molecule delivery tasks. This and other methods used for their modification make them much closer to organic NPs in terms of applications in neurobiology than to their close relatives, i.e., CNTs, which are utilized mostly for electrode manufacturing (see Section 5.6). Fullerenes can be functionalized to be biologically compatible while remaining resistant to metabolic degradation, which would cause the release of toxic metal ions into biological tissues. One application of water-soluble metallo-fullerene derivatives is their use as magnetic resonance imaging contrast and therapeutic agents, ${ }^{[245,246]}$ which are also applications of some inorganic NPs 
(see Section 4.2). Such systems have been used to image rat glioma cells in the brain, ${ }^{[247]}$ a very aggressive neural glial neoplasm associated with high morbidity and mortality. By functionalizing the surface of the fullerenes, it was possible to target the desired cell population and image at a concentration that was an order of magnitude lower than required by routinely used commercial contrast agents. ${ }^{[247]}$

Another potential application of fullerenes is neuroprotection, the pharmacological rescue of neurons before they die following disease or injury. This is a special property not ordinarily found in other organic or inorganic NPs. Following injury of neural tissue of brain or spinal cord, which can be exemplified by a trauma or a surgery related to implantation of an NE, the primary mechanical damage quickly initiates a complex chain of biochemical reactions which lead to the so-called "secondary injury" that includes biochemical processes such as free radical damage involving ROS and excitotoxicity produced by the spillage of neurotransmitters and excitatory ions. The effects of secondary injury damage can extend far beyond the locus of the primary mechanical injury, and result in the death of neurons relatively far away. Immediately adjacent to the region of cell death produced by the primary injury is a region referred to as the ischemic penumbra, an area where effected neurons can be rescued from cell death if pharmacological intervention is achieved quickly and efficiently. Clinically, neuroprotection is important because the rescue of even a small percentage of neurons can result in significant clinical recovery. Hydroxyl functionalized fullerenols have been shown to possess anti-oxidant and free radical scavenger properties which are neuroprotective. These corresponding fullerenes have been shown to result in significant cellular rescue in animal CNS injury models\{LL, 2001 \#551;Dugan, 1997 \#550;Dugan, 1996 \#553;jin, 2000 \#562\}. Free radical injury is a major contributor to the disease process of ischemic, traumatic, and degenerative disorders in the CNS, and is a major component of secondary injury mechanisms. ${ }^{[248-251]}$ ROS including superoxide $\left(\mathrm{O}_{2}-^{\circ}\right)$, hydroxyl $\left({ }^{\circ} \mathrm{OH}\right)$, peroxynitrite $\left(\mathrm{ONOO}^{-}\right)$, and peroxide $\left(\mathrm{H}_{2} \mathrm{O}_{2}\right)$ can result in DNA fragmentation, peroxidation of cell membrane lipids, decreased mitochondrial energy production, and transporter protein inactivation. Fullerenols have been shown to reduce and limit glutamate, NMDA, AMPA, and kainite induced excitotoxic and apoptotic cell death. ${ }^{[252-254]}$ Although still an area of active research, the mechanism of fullerenol-mediated neuroprotection is the result of, at least in part, their ability to inhibit glutamate channels, since GABA(A) or taurine receptors in neurons were not affected by the fullerene derivatives. Fullerenols also appear to lower glutamate induced increases in IC calcium concentrations, a prominent mechanism of neuronal excitotoxicity.

\subsubsection{Self-organized Scaffolds for Neuronal Guidance}

The replacement of neurons around NE with reactive astrocytes and glial cells can be combated by stimulation of neuronal growth toward NEs. Along with other means described above, this can be accomplished by using a specifically designed cellular environment, i.e., scaffolds. Bioactive and biocompatible peptide-based self-assembling nanofibers that form self-supporting scaffold materials are being developed for neural regeneration applications. ${ }^{[25-257]}$ These types of systems are attractive because they can mimic aspects of both the mechanical and biochemical properties of the extracellular matrix. In doing so, they can signal and induce very specific responses in neurons (e.g., chemotaxis axon growth, ${ }^{[258]}$ proliferation of neural progenitor cells, etc.). For example, ionic self-complementary peptides assemble because of the alternating positive and negative L-amino acids, which form highly hydrated scaffolds at physiological conditions. ${ }^{\text {[259-263] }}$ These nanofibers signal neurons at molecular scales while in a fully biocompatible system because the L-amino acids can be broken down and metabolized. It is also free of chemical and biological contaminants such as collagens, which are normally present in animal-derived biomaterials, and is immunologically inert. One study showed robust adhesion of PC-12 cells, a neuronal derived cell line, and functional synapse formation in rat primary hippocampal neurons with a arginine, alanine, aspartate, and alanine (RADA)16-I scaffold. ${ }^{[260]}$ Another study using this material facilitated the functional regeneration of axons in a hamster model of severed optic nerve that resulted in partial functional visual recovery. ${ }^{[259]}$

In another system, peptide amphiphile molecules were developed to self-assemble under physiological conditions into networks of nanofiber scaffolds. ${ }^{[255,256,264]}$ The nanofiber surface consisted of hydrophilic head groups of the peptide amphiphile molecules that had aligned to form elongated micelles expressing specific bioactive peptide sequences. In particular, the neuronal specific extracellular matrix laminin-derived sequence isolucinelysine-valine-alanine-valine (IKVAV) ${ }^{[257]}$ was used to promote the outgrowth and elongation of neurites, the processes extended by growing neurons. In water, the peptide amphiphile molecules energetically prefer to be in solution, while in the presence of cations, such as conditions found in physiological environments, self-assembly is triggered and the molecules form nanofiber scaffolds that trap the water molecules that surround them, macroscopically forming a weak self supporting gel. ${ }^{[258]}$ Because of the conditions under which self assembly occurs, mixing peptide amphiphile solutions with suspensions of neural progenitor cells or neural retinal cells in culture media resulted in their encapsulation in the three dimensional gels. Functional cell signaling was induced because of the structure of the nanofibers, and the measured response of the signaling was confirmed using different assays. The end result was selective and robust neuronal differentiation of the progenitor cells, with minimal astrocytic differentiation. ${ }^{[257]}$

\subsection{Inorganic Nanocolloids}

Another major class of nanomaterials being used for neuroscience applications are inorganic NPs, which form a diverse family of materials capable of being functionalized including silica NPs, ${ }^{[232]}$ semiconductor, metallic and magnetic NPs. ${ }^{[265]}$ Overall, inorganic NPs in conjunction with neuron tissues are used predominantly for the same tasks as organic cousins with a difference that drug delivery is a less prominent functionality, while imaging tasks are more predominant. Additionally there are specific properties of inorganic NPs which are not encountered in any other NP, which make possible unique neurological applications. ${ }^{[266-268]}$ 
Super paramagnetic iron oxide NPs which are also often abbreviated as super paramagnetic iron oxide nanoparticles (SPIONs), have been used in magnetic resonance imaging to detect neurodegenerative lesions with high sensitivity and efficacy. They are typically made from oxides of iron and manganese, ${ }^{[268]}$ but $\mathrm{Co} / \mathrm{Pt}$ or $\mathrm{Au} / \mathrm{Fe}$ alloys ${ }^{[269]}$ can also be considered. For neural applications, the presence of Co is undesirable because of neurotoxicity. Magnetic NPs are able to cross the blood brain barrier by active transport mechanisms ${ }^{[228,268,270]}$ or via the olfactory bulb. ${ }^{[271]}$ Polyvinyl-alcohol (PVA) and dextran coated magnetic NPs were investigated for uptake using brain capillary endothelial cells, microglia (the immune cells of the CNS), and three-dimensional cellular aggregates. ${ }^{[272]}$ These NPs did not induce the production of the inflammatory mediators, at least in their simple test systems. Amino PVA-SPIONs were taken up by isolated brain-derived endothelial and microglial cells with no inflammatory activation. One potential exciting and unique application of these NPs is targeted CNS drug delivery simultaneously combined with magnetic resonance imaging detection. ${ }^{[272]}$ Magnetic dispersions capable of strong luminescence emission were also made for better spatial resolution with magnetic fields. ${ }^{[273,274]}$

In terms of the neural interface, magnetic NPs are particularly attractive for treatment and imaging of brain cancer. ${ }^{[275-279]}$ In treatment modalities, the particles are used as a source of heat in AC magnetic fields, which raises the temperature of cancer cells and kills them (i.e., hyperthermia). ${ }^{[267,275]}$ Alternatively, they can be used for delivery of medical agents and targeted by external magnetic fields. ${ }^{[280]}$ Preliminary studies on the distribution of magnetic NPs in animals have also been done and successfully indicate crossing of the BBB. ${ }^{[14,281,282]}$

A class of NPs that is emerging as an important research tool is semiconductor NPs (see Section 3.1). The optical properties of quantum dots result in very specific and narrow excitation spectra induced by rather broad absorption spectra, especially toward the UV range. They are generally composed of a CdSe or CdTe core. As described in Section 3.1, they are often coated with a zinc sulfide cap to enhance their luminescence and to make them more biocompatible. By functionalizing their surface, they can be used to tag molecules of interest in biological systems. ${ }^{[283-288]}$ Quantum dots have been used to label and track both neurons and glial cells, the two major classes of cells in the CNS. ${ }^{[173,289]}$ One unique and technically very difficult application that this technology is particularly well suited for is the probing of individual receptors, such as neurotransmitter receptors. ${ }^{[290]}$ In neuronal chemical synapses, the space between the pre- and postsynaptic neurons where the presynpatic cell signals a postsynaptic one via neurotransmitter release, is only about $20 \mathrm{~nm}^{[291]}$ and molecularly very diverse and heterogeneous, making it an extremely difficult structure to study. The size, functionalization, and optical properties of quantum dots make them a potentially invaluable tool for this application.

\section{Surface Nanostructures and Neural Tissues}

Continuing the discussion about the interactions of nanomaterials and neural tissues following the previous sections on biocompatibility and nanoscale colloids, we can move on to the topic of how nanostructured systems can eliminate or mitigate the challenges of neural interface outlined in Section 2.3. Indeed, there is a significant success in all of the directions mentioned from improving the charge storage capacity, to single neuron addressing, to mitigation of inflammation including the techniques involving nanoscale colloids. In this section, we will describe approaches based on nanostructured surfaces, which are probably more direct and diverse compared to what we have seen so far in case of NPs and similar dispersions.

\subsection{Basic Electrode Design}

Cell organelles and structures responsible for the interface with $\mathrm{NE}$ span the range from nanometers to micrometers in size. ${ }^{[18]} \mathrm{As}$ was mentioned already, biological structures such as growth cone filopodia and extracellular matrix proteins lie within the nanometer range, ${ }^{[292,293]}$ as do many other relevant structures in the cellular environment. For example, basement membranes that provide the surrounding structure for most cells are composed of a dense meshwork of three-dimensional topography, featuring pores and fibers with dimensions ranging from tens to hundreds of nanometers. ${ }^{[294]}$ Therefore it seems logical to us that NEs should have scale of at least some features in the same nanometer range.

We will confine the discussion here predominantly to the nanoscale features of electrodes which have a solid interface with cells although some radical departure from the traditional electron-transporting electrodes should also be noted. One example of this type of a non-conventional electrode is the microfluidic-based stimulating electrodes, ${ }^{[295-302]}$ which can arrange the delivery of chemical signals to neuronal networks. Microfluidic systems have the important advantage of communicating to the neurons with a probably more appropriate "language" of chemical signals and ionic currents rather than electronic currents. However, they have intrinsic limitations as implantable devices with respect to liquid storage capacity and/or their external supply of the neurotransmitters. As an experimental tool for the ex vivo investigation of operations of neurons and their networks, ${ }^{[297,303]}$ microfluidic system can be exceptionally convenient, particularly when combined with nanoscale surface modification, ${ }^{[304,306]}$ and creation of 2D and 3D gradients of biochemicals affecting neuronal stimulation. ${ }^{[303,306,307]}$

\subsubsection{Nanoscale Electrodes and Inflammation Reactions}

Nanoscale NEs have the potential to circumvent reactive biological responses by virtue of their small size and mechanical properties (see Section 2.3.3). Specialized techniques resulting in microscale lesions ${ }^{[308,309]}$ have been successfully employed to minimize scarring due to acute trauma to the tissue and vasculature caused by implantation, ${ }^{[98]}$ and it is reasonable to postulate that these results will also apply to nanoscale implants. As for glial scarring due to chronic electrode presence, it is unclear what type of reactive response nanoelectrodes will elicit, but reasonable assumptions can be made. Decreased effects of electrode motion due to smaller electrode sizes and less disparity between mechanical properties of tissue and flexible nanoelec- 
trodes $^{[88,104]}$ should both contribute to a decreased reactive response to chronically implanted nanoelectrodes. ${ }^{[310]}$ Additionally, nanoscale probes have a unique potential to access neural tissue without interfering with normal physiological function. For example, proof-of-concept experiments with sub-micron wires have demonstrated that NW probes could be introduced via catheter into the microvasculature of the brain, where their small dimensions would allow them to innocuously record surrounding electrical signals without interfering with blood flow or normal brain activity. ${ }^{[52]}$

\subsubsection{Nanoscale Electrode Arrays}

Continuing the topic of nanoscale electrodes, arrays of NEs, similar to the MEA in Figure 1G, for example, will also benefit from reduction of size. Nanoscale NE array technology is an area of research still in its infancy. ${ }^{[311]}$ Many of the developments in the field have served to establish reliable processing techniques for nanoelectrodes. ${ }^{[311]}$ These advances have laid the groundwork for a wide range of future applications, but critical technical hurdles remain. One set of challenges is associated with the physical characteristics of nanoprobes and their need to have sufficient mechanical strength and toughness-characteristics that might not be attainable using classical materials.

The task of developing implantable nanoscale NE arrays containing dense collections of individually addressable nanoelectrodes involves even greater technological hurdles. As discussed earlier, decreased surface area of miniaturized electrodes results in dramatically increased electrode impedance. Additionally, the addressing of individual nanoelectrodes within large arrays poses a new set of challenges. Nanoscale wires and interconnects have been developed, but significant breakthroughs in these areas would be required to cope with the wiring complexities that would be associated with dense NE arrays. Notably, FETs and NW circuits offer alternative approaches for two-way communication with neurons (see Sections 5.7 and 5.8).

\subsection{Nanoscale Patterning of Electrodes}

\subsubsection{Chemical Patterning}

Recent advances in nanotechnology allow one to control neural interfacing through nanoscale chemical and physical patterning. The latter refers predominantly to topography and is considered in the subsequent section, whereas the former encompasses a variety of cell-adhesive and cell-repellent layers. All of these strategies can be useful tools in fighting the immune response, neural depletion around NEs, and, at the same time, make possible the creation of sophisticated NE-live cell systems and unique neural devices.

A variety of surfaces have been designed to reduce cell adhesion, including those modified with self-assembled monolayers (SAMs) presenting hydrocarbons, ${ }^{[312]}$ mannitol, ${ }^{[313]}$ PEG,$^{[314-316]}$ oligoethylene glycol $(\mathrm{OEG}){ }^{[315-317]}$ and fluorinated hydrocarbons. ${ }^{[318-320]}$ One critical issue is that non-polar surfaces that inhibit cell adhesion will bind to hydrophobic domains of soluble proteins and thus may become less effective over time. ${ }^{[315,317]}$ Other surfaces, such as PEG, resist both cell and protein adhesion but can autooxidize, especially in the presence of the transition metal ions that are at high concentration during cell culture. ${ }^{[315]}$ Fluorinated hydrocarbons present an effective inert surface in conditions that do not contain protein-rich serum because (i) they are among the most hydrophobic surfaces achievable by a $\mathrm{SAM}^{[318]}$ and (ii) are quite stable under culture conditions. ${ }^{[319,320]}$ OEG surfaces are also relatively stable and have been shown to resist cell spreading in protein-rich conditions for over 1 week in culture. ${ }^{[313,316]}$

Cell-adhesion coatings include factors such as poly(lysine), ${ }^{[179,320]}$ poly(lysine)-conjugated laminin, ${ }^{[321]}$ fibronectin, ${ }^{[322]}$ and collagen, ${ }^{[323]}$ which can be patterned with nano- and micron-scale resolution using photolithography or microcontact printing. ${ }^{[324,325]}$ Such patterned surfaces have been used to direct the attachment and confined growth of neurons, as well as cardiac myocytes, fibroblasts, and HeLa cells.

Critical to effective cell patterning is choosing a matrix that allows cells, often patterned at low densities, to remain viable in culture and retain the electrophysiological properties exhibited in bulk culture. In a recent study, ${ }^{[320]}$ sparsely plated neurons were cultured on patterned polylysine, with neurite growth guided along micropatterned lines, and several key observations were reported. First, neurons were shown to be viable for up to 17 days while maintaining a resting potential and membrane time constant similar to neurons grown in standard culture conditions. Secondly, patch clamp electrophysiology was used to demonstrate that patterned cells exhibit electrophysiological properties similar to those that are not patterned. Thirdly, both inhibitory and excitatory neurons were present in patterned networks and were shown to form interconnected networks with functional synapses. Finally, given sufficiently long culture times, the synapses matured and exhibited spontaneous and evoked synaptic currents. Taken together, these studies ${ }^{[320]}$ indicate that patterning of adhesion-promoting material presents a general platform for cell patterning, and specifically, that patterning of poly(lysine) enables the culture of low-density neurons and neural networks with guided neurites that exhibit the physiological properties of non-patterned neurons.

\subsubsection{Topography}

Topographic effects have been investigated extensively for silicon and titanium, ${ }^{[326]}$ and to a lesser extent for polymers, such as poly(methylmetacrylate). ${ }^{[327]}$ Silicon in particular is commonly used because of well established lithography tools and protocols, previously developed for electronics. Methods of patterning of organic materials, including ink-jet printing, nanoscale imprinting, and PDMS stamping, were developed more recently.

Physical patterning to provide topographic cues offers a potent means for the control of cell adhesion, migration, orientation, shape, and even gene expression. ${ }^{[327-336]}$ Though surface topography and chemistry interact in a complex synergistic manner, ${ }^{[337,338]}$ topographical cues alone ${ }^{[339]}$ are able to exert considerable influence over the cells they contact. ${ }^{[257,326,340,341]}$ Topographic stimuli appear to affect both neural and nonneuronal cell types, and different topographies exert varying effects on adherent cells. ${ }^{[294,328,330-335]}$ An approach toward topographic patterning that often proves effective is to mimic the 
physiological structures that normally interact with the cell type of interest.

In the simplest case, nanotopography can be altered by randomly modulating a substrate's surface features (i.e., varying its roughness). Modulation of surface roughness is technically straightforward, and has been applied to enhance the function of many biomedical materials, including silicon, silica, titanium, titania, stainless steel, iridium, iridium oxide, and a variety of plastics used in neural medicine and NEs. ${ }^{[217]}$ Chemical etching of materials is one method that can be used to pattern surfaces with roughness at the nanoscale, and has been used to promote cultured cell adhesion and viability. ${ }^{[342,343]}$ Reactive ion etching has also been used to create randomly patterned Si column structures that promoted astrocyte adhesion. ${ }^{[34]}$ Strong neural attachment to implanted electrodes is desirable as it increases interface stability and improves electrical transfer across the tissue/electrode boundary. ${ }^{[343]}$ A plausible mechanism by which nanoscale surface roughness promotes cellular adhesion is through increased contact area resulting from cell membranes conforming to fit the roughened surface. ${ }^{[342,343]}$ An "average roughness" of 20-70 $\mathrm{nm}$ was found to be optimal for promoting neural adhesion and survival on etchant roughened $\mathrm{Si}^{[342,343]}$ Similar metrics for other substrate materials and cell types have yet to be established. It should be noted, however, that roughness levels are somewhat difficult to define and quantify, ${ }^{[326]}$

To enable the patterning of neural systems with greater complexity than a monolayer of randomly connected cells, a technique for achieving more controllable nanoscale patterning is often preferred. Grooved topography is one of the most popular methods for controlled patterning of neuronal cells due to well developed nanolithography capabilities, and because directed cell growth is necessary for a variety of NE applications. ${ }^{[326,339,345]}$ Neurons tend to attain an elongated shape within grooves due to surface-induced rearrangements of the cytoskeleton. ${ }^{[346]}$ Neural growth cones are very sensitive to physical topography, even in the absence of specific biochemical cues, and this sensitivity can be exploited to achieve directed growth. It was found that the majority of cells closely follow grooves and ridges, even over discontinuities. ${ }^{[327]}$ For example, axons of adult mouse sympathetic and sensory ganglia were shown to display pronounced changes in the orientation when cultured on horizontally imprinted patterns (Fig. 7A and B). Different patterns consisting of parallel grooves with depths of $300 \mathrm{~nm}$, widths of $100-400 \mathrm{~nm}$, and distances between two adjacent grooves of 100-1600 nm were investigated. The influence of these patterns appears to be largely a function of the relation between axon diameter and groove/ ridge width. Axons of peripheral neurons experience the guiding effect by topographic patterns when the lateral features are $100 \mathrm{~nm}$ or larger, which is important to consider when dealing with patterns made by aligned nanomaterials of smaller diameters, for instance SWNTs. ${ }^{[18,19,206,347,348]}$ One may also note that the nerve cell processes preferred to grow on ridge edges and elevations in the patterns rather than in grooves (Fig. 7C). This seemingly claustrophobic behavior is potentially explained by the better nutrient delivery conditions in these locations.

It is interesting to compare the guidance effects of nanoscale topographical features with those exerted by chemical patterns. ${ }^{[337]}$ When a range of groove depths from nanometric up to
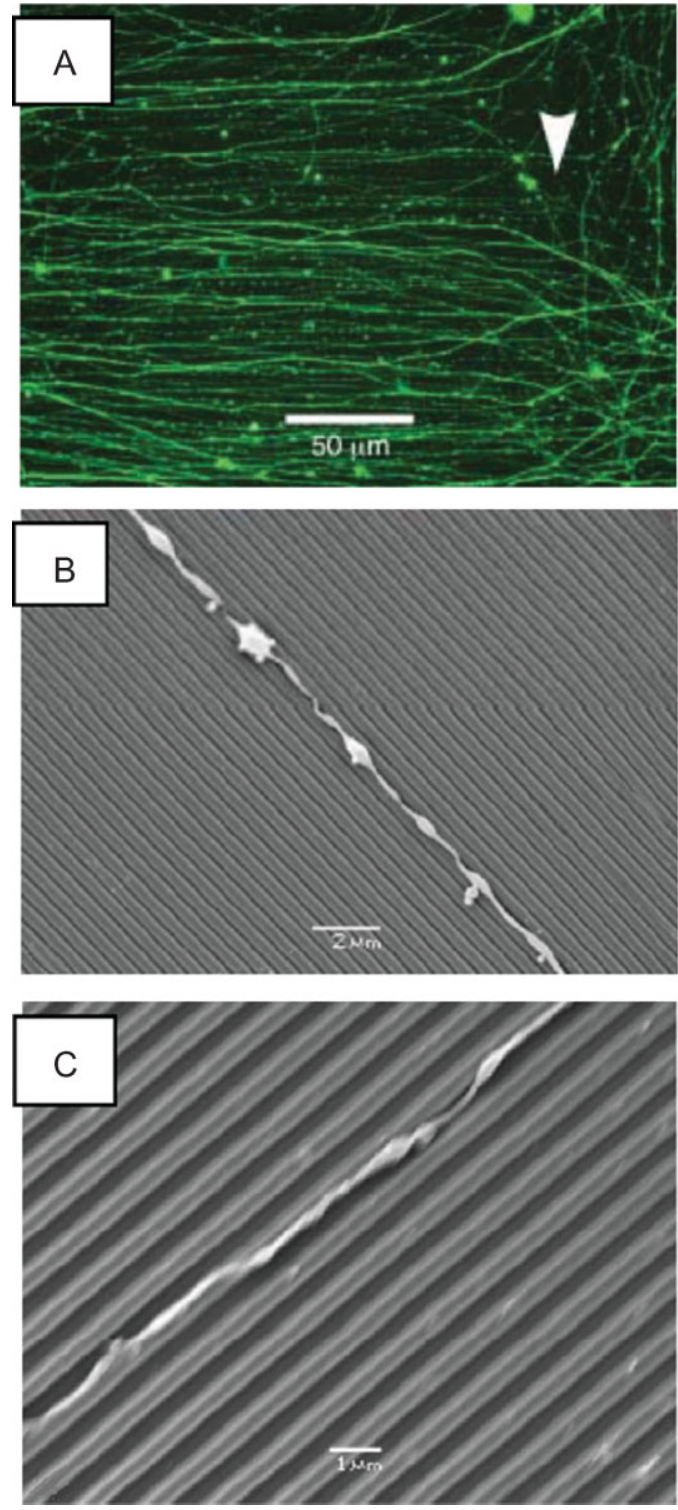

Figure 7. Alignment of axons of adult mouse sympathetic and sensory ganglia on horizontally imprinted pattern made by nanoscale contact printing with different topology. A) $200 \mathrm{~nm}$ width and $400 \mathrm{~nm}$ pitch. The arrow indicates the border of the pattern. The more random growth of axons outside the pattern is clearly visible. Note that the larger axons are not as well guided as the thinner ones. B) $100 \mathrm{~nm}$ width and $500 \mathrm{~nm}$ pitch. C) $400 \mathrm{~nm}$ width and $800 \mathrm{~nm}$ pitch. SEM images showing that the axons grow on the ridge edges and not in the grooves. Adapted with permission from [327]. Copyright 2000, Blackwell.

micrometric was used, it was found that the deeper the groove the more pronounced were the effects of the topography, so that at the greatest depths the nanoscale topographic groove effect dominated the chemical track effects. ${ }^{[337]}$

Considering the potent effects of topography on cellular behavior, it is no surprise that gene expression is affected by the nanoscale features of underlying surfaces. ${ }^{[349]}$ The largest study in this area was carried out using gene arrays and reported that the expression of different genes was increased and decreased as 
a result of growing fibroblasts on pillar nanotopography. ${ }^{[292,350-352]}$ If this finding applies to neurons, then topography represents a potentially powerful system for controlling the phenotype of cells. It is possible that nanoscale topological surface features induce changes in chromosome territories. ${ }^{[350]}$

While guidance of axons and neurites by surface topography and chemistry is feasible and effective, it is perhaps physiologically more relevant to affect neural guidance via alignment of supporting cells. Longitudinally oriented glial cells stimulate and support the parallel growth of axons and neurites ${ }^{[353,354]}$ not only via physical guidance cues, but also through aligned and constrained pathways of biochemical guidance cues, such as secreted ECM molecules. ${ }^{[35]}$ Fibroblasts, meningeal cells, astrocytes, and Schwann cells, oriented on substrates with topographically aligned features, have all been shown to successfully guide neurite and axonal growth. ${ }^{[338,353,355-357]}$

\subsubsection{Additional Techniques for Topographic Patterning for Neural}

Tissues

Most studies on the effects of topography on neural tissues have been performed on substrates patterned with traditional photolithography (see Section 5.2.2). Several additional techniques, however, each with their own tradeoffs, can be used to achieve organized nanoscale patterning of a substrate. Electron beam lithography (EBL), for example, offers precise individual features, as small as $3-5 \mathrm{~nm},{ }^{[358]}$ but this resolution comes at the cost of expensive equipment and slow processing. ${ }^{[359]}$ Furthermore, to fabricate patterns across larger surface areas, minimum feature sizes increase up to around $30 \mathrm{~nm}^{[358]}$ For reference, fibroblast filopodia have been shown to respond to nanoscale patterns as low as $35 \mathrm{~nm} .{ }^{[292]}$ As a step toward cost efficient mass production, there are current efforts to replicate EBL patterns by employing molds and nanoimprint lithography. ${ }^{[292,359]}$

A wide variety of simpler and more cost-effective techniques of achieving controllable nanoscale substrates are also available. Technologies such as dip pen nanolithography ${ }^{[360]}$ and electrostatic atomic force microscopy ${ }^{[361]}$ can be used to deposit or detect individual molecules to pattern and to characterize nanoscale materials. Neural cells have been cultured on surfaces patterned by these approaches with true nanoscale features, and specific cell adhesion and growth patterns were shown to result within specific ranges of nanoscale physical structures. ${ }^{[342,362-364]}$

Simpler technologies for nanoscale patterning exists as well, but they typically come at the cost of specific feature geometries. ${ }^{[359]}$ For example, the technique of electrospinning can be used to create aligned layers of polymer fibers of nanoscale diameter. ${ }^{[365]}$ Briefly, a high voltage is applied between the tip of a syringe as it slowly ejects a liquid polymer melt and a collecting surface. The collection of charge on the syringe tip causes repulsive forces resulting in the ejection of "nanofibers" that are projected toward the collecting surface. One method of achieving alignment of the nanofibers is to use a high-speed rotating disk or drum as the collecting surface. The resulting aligned nanofibers have been shown to direct glial alignment and promote directed growth of neurites and axons (Fig. 8). ${ }^{[366-369]}$ Aligned nanofiber scaffolds are thought to mimic the structure of collagen, a major component of extracellular matrix, by creating fibrous networks that provide structural strength and support cell growth. ${ }^{[359]}$

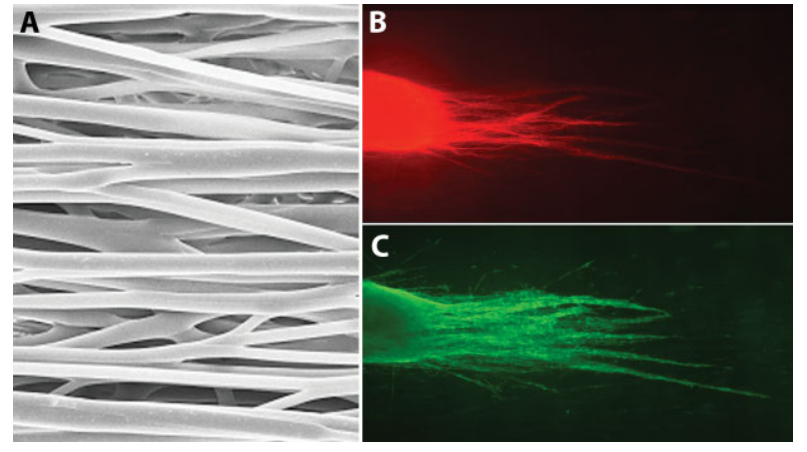

Figure 8. A) SEM image of aligned poly acrylonitrile-co-methylacrylate (PAN-MA) nanofibers, B) NF-160 staining of neurite outgrowth from a dorsal root ganglion (DRG) cultured on an aligned nanofiber film, C) S-100 staining of Schwann cell migration from the same DRG. (Courtesy of I. Clements, V. Mukhatyar, and R. Bellamkonda, Georgia Institute of Technology, Atlanta, USA).

Several types of regeneration scaffolds employing electrospun nanofibers at their core have been shown to effectively facilitate nerve regeneration in vivo. As an example, one such construct ${ }^{[370]}$ consists of a semipermeable tube containing stacked film layers of aligned nanofibers, and has been shown to promote axonal regeneration with an effectiveness approaching that of autografted nerve segments, the current clinical gold standard. ${ }^{[371]}$

\subsection{Model System for Replication of Neural Networks}

Understanding of information processing in neural networks and tissues is critical for understanding mechanisms of treatment and designing more efficient NEs. For that reason, it would be of value here to discuss briefly some simple model systems to study signal processing in neuron networks. They can be quite interesting from the perspective of utilization of nanotechnology capabilities in fundamental neurology. Incidentally, they are also intimately related to the patterning of electrodes with nanoscale features described in the previous section.

The simplest models are based on geometrical patterns of neurons created on substrates using chemical and/or topographical cues presented above. Starting with predominantly microscale patterns, poly(dimethylsiloxane) molds have been used to study neuron-astrocyte communication by designing experiments that control when and how cells grow and signal each other, which allows dissecting apart the molecular details of complex signaling. ${ }^{[372-375]}$ By plating neurons in one well and neural astrocyte cells in adjacent wells, chemical signaling via soluble factors that diffused from well to well could be studied. ${ }^{[372-375]}$ Similarly, by creating specific patterns using substrates on which cell processes can grow, and discouraging cell growth outside of these patterns using materials which cells cannot adhere to, hypotheses about the cellular mechanisms of neuronal signaling have been successfully investigated. ${ }^{\text {[372-375] }}$

Using the guiding effect of topographic features, one can create fairly complex patterns of neural cells. ${ }^{[376,377 a]}$ Particularly fascinating are the patterns where the cells are interconnected with each other in a controlled fashion represented by Figure 9G, which can be considered as prototypes of (i) much simplified ex 


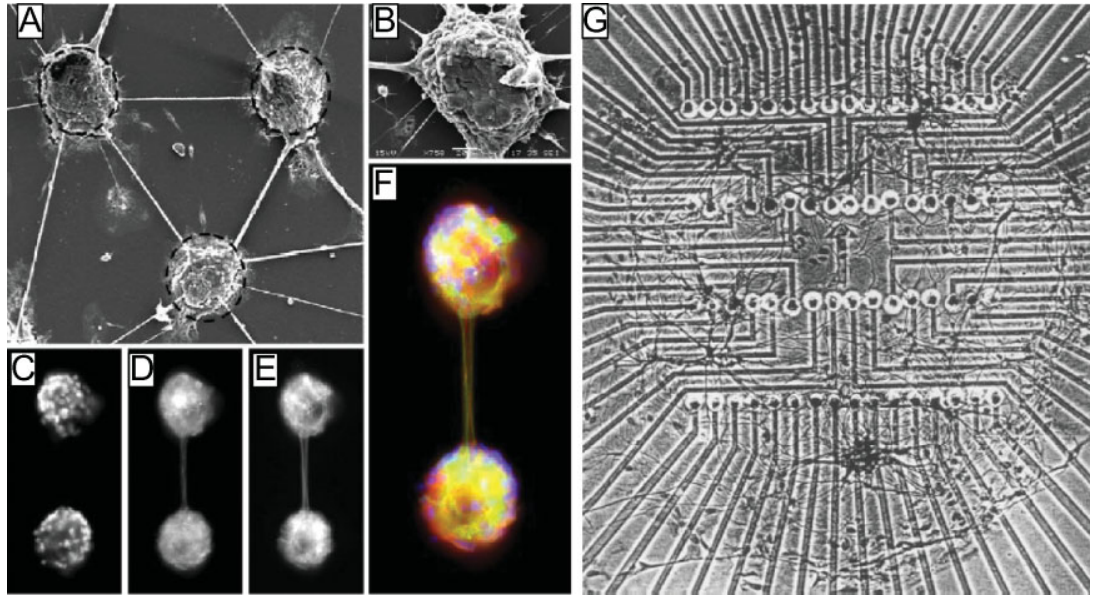

Figure 9. A) A SEM image of an engineered neural network. The dashed circles mark the polylysine islands used to define the network. B) Each of the cell clusters contains hundreds of cells and extends between 1 and 8 bundles. C-F) Multiple staining of two interconnected clusters. C) DAPI, cell nuclei. D) NFM, the bundle connecting them is composed of axons and dendrites. E) MAP2, neurons. F) red, axons; green, dendrites; blue, cell body. The yellow color arises from coincidence of green and red fluorescence (from [336]). G) Neuronal network made from 76 neurons growing on the matrix of standard 64 electrodes. Adapted with permission from [377b]. Copyright 1994, Academic Press.

vivo models of neural networks existing in neural tissue and (ii) a network of NE pads with single cell excitation capabilities. The ex vivo models can serve both for understanding of signal transduction, stimulation effects (see Section 2.3.5), and drug discovery. It should be appreciated that though the growth cone(s) may conform well to the substrate, the axon or dendrite following behind may adhere poorly to the substrate. So, if the extension is mechanically strained by the route followed, as for example by attempts to turn acute angles, the main part of the neurite may contract and pull the neurite into the shortest path. In some instances, even the cell body may be detached and the cell is then left "suspended" between two or more growth cones or a growth cone and the cell body. For these reasons, the most stable patterns of neurons are orthogonally or hexagonally intersecting paths. Such considerations might account for the preference of axon extension or bifurcation to be about $60^{\circ}$. Other patterns might be sufficiently stable mechanically, but do not seem to have been used. The $60^{\circ}$ and related pattern represents a rudimentary model for information flow and processing. ${ }^{[378]}$

Patterning of neurons in 2D networks can be achieved via anchoring of cell clusters at specific positions without any interconnects between the islands or pads. ${ }^{[36]}$ The compact connections among pairs of islands occur spontaneously through a single non-adherent straight bundle (Fig. 9). The $100 \mu \mathrm{m}$ in diameter islands that stabilize the location of cell clusters can be both chemical and topological. The islands and pads were made of poly-D-lysine or CNTs, respectively. Multiple staining indicates (Fig. 9C-F) that there are no cell bodies in the interconnecting links. ${ }^{[336]}$ Monitoring the dynamics of the forming networks in real time revealed that the organization of the neural network is mainly driven by the ability of the neuronal cell clusters to move away from each other while continuously stretching a neurite bundle in between. The resulted network patterns are very stable and can be maintained for as long as 11 weeks. ${ }^{[336]}$
Although neural networks with square, triangular and circular geometry were realized, ${ }^{[336]}$ not only the size $(x, y$, and $z$ dimensions) of the nanoscale features but also the array pattern (i.e., orthogonal, hexagonal and random and other patterns) affects adhesion. ${ }^{[379]}$ It appears that cells prefer a pattern of particular symmetry. Though such tests have not yet been done on neurites, it is almost inherently obvious that there will be such effects because the fine processes conforming to the packing and spacing of the array are of similar size to the pillars (or pits). This suggests that misalignment of the arrays will lead to unintended routings, but it also indicates that the alignments of these NPs and related nanoscale features could be used to construct complex and precise nerve networks in the model neural structures.

\subsection{Drug Delivery Layers on Electrodes}

Biochemical coatings on the surfaces of NEs allow for modulation of the local tissue response while improving cell attachment and electrode performance. Dexamethasone, for example, is a synthetic glucocorticoid hormone that has been coated on implantable electrodes to reduce glial scarring and neuronal loss. ${ }^{[380-383]}$ Coatings not only enable precise localization of drug release, but can also offer sustained release over time. New techniques in nanotechnology allow for the fine control of coatings with thicknesses down to the nanoscale. These capabilities are important for fine-tuning drug delivery, and also for decreasing the overall thickness of coated probes in order to minimize the inflammatory response. ${ }^{[12]}$

Layer-by-layer (LBL) assembly is an example of a process that can be used to produce film coatings with precise thicknesses on the order of nanometers. ${ }^{[384]}$ Briefly, LBL assembly exploits secondary interactions between macromolecular species to sequentially build the coatings one nanometer layer at a time. The most common realization of LBL is the combination of two oppositely charged polyelectrolytes. ${ }^{[385]}$ Cyclic repetition of adsorption steps using polyelectrolytes of alternating charge can be used to yield multilayered structures of arbitrary thickness. ${ }^{[384]}$ Due to the fact that these processes are largely unconstrained by the nature, size, or topology of the substrate, ${ }^{[384]}$ LBL assembly is highly versatile and has been used to deposit bioactive coatings on implantable electrodes for a wide range of biosensing applications, ${ }^{[386]}$ for electrical neural interfacing, ${ }^{[16,183,203]}$ and recently, with neural stem cells (NSCs). ${ }^{[387]}$ For example, nanoscale coatings of laminin on silicon wafers have been shown to enhance attachment and differentiation of cortical cells cultured on the wafer surface. ${ }^{[385]}$ In another set of experiments, chronically implanted silicon microelectrode arrays coated with nanoscale laminin layers were shown to elicit a decreased gliotic response after 4 weeks in vivo. ${ }^{[388]}$ Significantly, the integrity of these coatings was not compromised by the shear forces encountered during electrode insertion. Furthermore, the 
LBL coatings had no observable effect on electrode impedance around the biologically relevant range of $1 \mathrm{kHz} \cdot{ }^{[385]} \mathrm{LBL}$ techniques have also been used to embed anti-inflammatory agents such as piroxicam and $\alpha$-MSH. ${ }^{[389,390]}$

\subsection{Electrical Coatings on Electrodes}

Nanostructured materials can provide an excellent foundation for NE design focused on improving charge storage capacity and reduction of electrode size. In the realm of organic nanomaterials, the most significant strides in this direction were made with conductive polymers, ${ }^{[391]}$ exemplified by poly(3,4ethylenedioxythiophene) (PEDOT). Through electrochemical deposition, these conductive polymers can be precisely localized to active electrode sites with coating thicknesses down to the nanoscale. ${ }^{[392,393]}$ As compared to purely metallic electrodes, ${ }^{[112,394-401]}$ electrodes coated with conducting polymers provide a more adaptable interface with neural tissue with smaller hardness mismatch. The best examples of polymercoated electrodes made from poly(pyrrole) (PPy) or PEDOT can also significantly decrease the impedance of microelectrodes at $1 \mathrm{kHz}$ by about two orders of magnitude and increase the charge transfer capacity of microelectrodes by three orders of magnitude in comparison to bare Au electrodes. ${ }^{[357,370,392,402]}$ Although Au electrodes do not have the best electrical characteristics as NEs (see Section 2.2), and in fact, far inferior in respect to charge injection capacity to other materials, they are often used for comparative reasons due to simplicity of preparation and availability. To a large degree, the improvements mentioned above are attributed to the NT morphology of this material (Fig. 10), which is molded by sacrificial electrospun fibers that are subsequently dissolved after coating with PPy or PEDOT. (Later similar morphology can be seen in CNT electrodes (see Section 5.6).) It is still difficult, however, for PPy and PEDOT to exceed charge injection capabilities of $\mathrm{IrO}_{x} \cdot{ }^{[403]}$ Additionally, the conductivity of conducting polymers is not as high as one would want it to be $\left(300 \mathrm{~S} \mathrm{~cm}^{-1}\right)$ comparatively to metals and they have to be used on conductive (metallic) substrates. So, the same issue of mechanical mismatch and likely activation of reactive astrocytes by micromotions of the electrode are still present. Improvement of the electrical characteristic of the immediate interface between the electrode and cells is important, but it will not benefit the long-term implants unless a solution for electrode encapsulation is found. This holds true for stimulation of deep brain areas, spinal cord, the eye, and peripheral nerves.

Additionally, conductive polymer coatings can be doped with biological agents to minimize tissue reaction and promote neural growth and adhesion. NGF and extracellular matrix proteins, for example, have been localized directly to electrode sites to enhance neural growth and adhesion. ${ }^{[357,404,405]}$ Furthermore, biological agents can be incorporated into the conductive coatings such that their release can be controlled over time via electrical stimulation. ${ }^{[392,406]}$ In one study, for example, an anti-inflammatory agent was mixed within a degradable polymer that was electrospun into a 3D structure of nanoscale fibers on the surface of a neural probe. ${ }^{[392]}$ Conductive polymer was then deposited to coat this 3D nanotubular structure, and subsequent electrical stimulation via

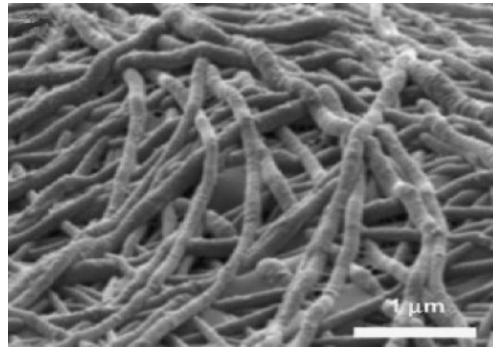

Figure 10. SEM image of NE from conducting polymer. Reproduced with permission from [394]. Copyright 2001, Elsevier.

the conductive polymer caused it to contract, expelling the anti-inflammatory agent in time-controlled bursts.

\subsection{Carbon Nanotube Materials for Neural Electrodes}

Many challenges facing current NEs (see Section 2.3) are related to mechanical and electrical properties of the electrode materials. The extraordinary strength, toughness, electrical conductivity, and surface area of CNTs make them excellent candidates for interfacing with neural systems for the development of biocompatible, durable, and robust neuroprosthetic devices. Mechanical compliance of highly conductive CNT composites with tissue can substantially reduce micromotions, suspected to activate the resident immune cells in neural tissues, and hence resulting in glial scarring (see Section 2.3.3). High surface area can drastically increase charge injection capacity ${ }^{[204]}$ and decrease the interfacial impedance with neurons, which is exactly what further miniaturization of electrodes requires. Smaller size of NEs will also reduce inflammatory response. Besides that, CNT coatings can also reduce delamination of electrodes from their underlying substrate, removing one of the significant technical issues with $\operatorname{IrO}_{x}$ electrodes. The same materials can also function as FET elements in the structures similar to those manufactured from Si described in Section 5.7 Additionally, the abundance of CNT derivatives involving biological components, such as peptides, ${ }^{[407,408]}$ proteins, ${ }^{[408-411]}$ and DNA ${ }^{[408,412-414]}$ also allows one to consider them as a potential player in drug delivery and treatment of CNS disorders, ${ }^{[154,188,190,415-419]}$ performing to some degree the functions of organic nanostructures described above.

The current state of the development of CNT/neural interface is far from the complete realization or even understanding of promising properties of NTs in respect to NE engineering. In 2000, it was suggested that CNTs could be used as substrates for neuronal growth. ${ }^{[151]}$ Scanning electron microscopy (SEM) was used to identify the morphological changes of neurons brought about by MWNTs. The neuronal bodies were found to adhere to the CNT surface with neurites extending through the bed of CNT and elaborating into many branches. The neurons remained alive on the NTs for at least 11 days, and it was shown that several chemical manipulations on the MWNT enhanced neurites growth and branching. After this first report, several groups developed methods of neuron culture on CNT films. ${ }^{[16,19,152,200,202,420]}$ 
A similar study was carried out with living neurons on NT films monitored using traditional fluorescence techniques. ${ }^{[200,202]}$ The numbers of neurites were counted depending on the nature of the NT and their functionalization. Using hippocampal neuronal cultures, neuronal growth was systematically controlled by functionalizing MWNTs with different chemical groups and the morphological features of living neurons were characterized. Using these CNTs as scaffolds for neuronal growth, it was found that neurons grown on positively charged MWNTs showed more growth cones together with more successful branching, thus simple variations in electrostatic charge on MWNT could manipulate neuronal growth patterns. A related question is whether CNTs, simply due to their intrinsic structure, can directly improve the functional rewiring of disconnected neuronal networks.

The simple CNT coatings made by drying or growth in vacuum used in previous studies are intrinsically fragile. They are likely to degrade and delaminate over time, resulting in the same issues as currently used electrodes if not more severe ones. The first steps toward utilization of both electrical and mechanical properties of SWNTs in neuroprosthetic devices were made with NTs films assembled using the $\mathrm{LBL}^{[16,19,203,385]}$ or other methods of making NT composite coatings with considerable adhesion to the substrate and NT content significantly above the percolation limit. The layering with polymers method allows one to control the structure of CNT-polymer composites and incorporate both high conductivity and high strength in one material. ${ }^{[34,421-423]}$ The SWNT substrate was prepared by LBL layering with a negatively charged polyacrylic acid polymer (PAA) and SWNT coated with positively charged poly $(N$-cetyl-4-vinylpyridinium bromide-co- $\mathrm{N}$-ethyl-4-vinylpyridinium bromide-co-4-vinylpyridine). ${ }^{[19]}$ It was demonstrated that cell cultures of NG108-15 effectively grow and proliferate on these substrates. Moreover, the number of neurites spun from individual cells exceeds those developed on traditional cell growth substrates.

The next advance in neuron-NT characterization came from the measurement of spontaneous postsynaptic currents (PSCs) from a single neuron. ${ }^{[152]}$ The appearance of PSCs provides clear evidence of functional synapse formation and is a widely accepted index of network efficacy. The NTs used for neuronal growth were first functionalized and then deposited from a dimethylformamide solution; after evaporation, the NTs were defunctionalized by thermal treatment leading to glass slides covered by a film of NTs. Hippocampal neurons were attached and cultured on purified MWNT. This strategy allowed a long term and stable retention of films of CNTs on glass and, moreover, a long term neuronal cell culture. Hippocampal neurons grew and attached to the purified CNT film and extended several neurites, forming a net morphologically similar to that developed under control (CNT-free) growth conditions in terms of cell numbers and neural processes, as verified by scanning electron microscopy and immunocytochemistry. Neuronal cell bodies displayed normal morphology and dimension as well.

The focus of that investigation was then shifted to the functional properties of brain circuits grown integrated to a CNT substrate, by performing single-cell patch-clamp recordings. By this technique it is possible to monitor single-cell and network generation of electrical activity. Since neuronal activity is ultimately electrical activity, electrophysiological recordings allow monitoring of the efficacy of a brain network in processing information. The appearance of synapses, revealed by PSCs generation, provided clear evidence of functional network formation. Under these experimental conditions, the CNT substrate strongly increased the average PSCs frequency together with the frequency of spontaneous action potential generation in all recorded neurons when compared to control ones. Thus, growing neuronal circuits on a CNTs platform promoted a significant increase in brain network operation. Such an effect was not related to CNTs increasing the number of surviving neurons, suggesting that enhanced network activity was apparently related to a potentiated synaptic function more than an increase in the number of synapses.

Recent reports indicate the possibility to directly stimulate isolated neurons via CNTs in culture. ${ }^{[16,206]}$ In 2008, the same observations were made in primary cell cultures, and actual tissues of rats and monkeys. ${ }^{[420]}$ It was also indeed demonstrated that coating of metal electrodes with CNTs led to low impedance electrodes, ${ }^{[420]}$ however little or no explanation in this work was given about origin of such effects. Little is known about the electrical features of such a coupling, or the possibility to evoke, via purified CNT substrates, synaptic activity in long term neural circuits. The early studies indicated that extrinsic electrical stimulation through the SWNT induced the appearance of cell inward currents that were indistinguishable from those elicited via direct IC application of voltage steps. ${ }^{[16,206]}$ In SWNT-polyelectrolyte coatings, the stimulation of inward currents with a cathodic extrinsic stimuli suggested that cells were activated from current leakage into the bath via a resistive coupling to CNTs. Electrophysiological measurements indicated electrical excitation of neurons when current was passed through the SWNT coating, not distinguishable from traditional neuronal excitation associated with opening of classical voltage-gated cation channels.

Additional work ${ }^{[208]}$ employed electrophysiology and computational modeling in order to understand the nature of electrical coupling between neurons and pure SWNTs. The recordings suggested that coupling between neurons and SWNTs might in fact be at least in part resistive; further studying involved mathematical modeling. This combined approach implicated that any resistive coupling between bio-membranes and SWNTs is qualitatively indistinguishable from a coupling between SWNTs and the patch-pipette through the patch-seal path to ground. ${ }^{[208]}$ Thus, whole-cell patch clamp recordings from neurons stimulated by SWNTs may yield deceiving results. Hence, due to the non-idealities of the single electrode voltage clamp, eliciting $\mathrm{Na}^{+}$-currents in neurons through SWNTs stimulation does not conclusively prove a resistive coupling between SWNTs and neurons. Rather, this can be accomplished by detecting synaptic responses, such as evoked by action potentials elicited in non-clamped neurons using the electrical stimulus delivered via SWNTs. ${ }^{\text {[208] }}$

We need to mention that, electrical properties of SWNTs make them also suitable for capacitive stimulation of neurons and their functionalization may be instrumental in establishing cell/ surface interactions affecting the coupling mechanism. Capacitive coupling, in our opinion, will be a more efficient way to excite neurons more conductively with long-term function of implantable NE. 
Another recent development in CNT-based devices is represented by the design of multi-electrode arrays (MEA) to both electrically stimulate and record from neurons. Such MEAs were made by synthesizing islands of high density CNT on lithographically defined, conductive substrates. ${ }^{[424,425]}$ Both multiwalled and single walled CNT structures are being used as microelectrode neural interfaces following chemical functionalization. ${ }^{[204,258,426]}$ For example, proof of concept stimulation of hippocampal neurons has been demonstrated using vertically aligned hydrophilic functionalized CNTs electrode arrays. ${ }^{\text {[204] }}$ Advantages of MEAs over the traditional metallic ones are manifold and were, by and large mentioned when discussing normal electrodes. CNTs can drastically increase the charge injection capacity while further reducing the size, reduce inflammation, and provide uniquely biocompatible surface. However, we also have a concern over the mechanical integrity of microelectrodes at the point where CNTs are growing from the substrates. The forces applied by the cells during the micromotions to these points are expected to be quite large, which can threaten the integrity of MEAs and generate debris around the electrode.

\subsection{Interface of Traditional Field Effect Transistors and Neuronal Cells}

Starting from the 1950 s advances in neuro-electronic systems were related to the progress in electronics industry and circuitry processing. To a large degree, one can trace this trend in the utilization of silicon etching technologies and lithographic techniques to the development of Michigan and Utah electrodes (Fig. 1). Their success is based entirely on the excellent degree of control achieved for silicon processing during the development of electronic circuitry and MEMS devices.

In that respect, one of the microelectronic devices that attracted the most attention in the last decade for neuronal interfacing is the field-effect transistor (FET). FET devices for neural interfacing were initially developed to enable the study of signal processing in arborized neurons and neural networks. ${ }^{[427]}$ One of the main obstacles during the course of development of intracellular (IC) recording techniques of electrical activity in neurons was the simultaneous recording of signals from multiple neurons. Whereas voltage-sensitive dyes ${ }^{[428]}$ allow rapid changes in membrane potential of neurons to be detected and measured from multiple sites, selective measurements are impossible and the toxicity of the dyes, when illuminated, make them unsuitable for long-term recording. ${ }^{[377,429]}$ IC recordings with glass pipette electrodes and the patch-clamp method measure potential by penetrating the cell membrane with a micropipette to create a connection with low electrical resistance. ${ }^{[40]}$ Not only is this method invasive, but the contact between cell and electrode is difficult to maintain longer than several hours, needless to mention the difficulty for attachment of multiple electrodes. ${ }^{[431]}$ With the arrival of a complementary system consisting of a microscopic silicon element with perfectly insulating silicon oxide, it became possible to stimulate a neuron extracellularly by capacitive coupling through a silicon chip in 1995. ${ }^{[432]}$ The development of the FET as a neural interface device was predated by the manufacturing of sophisticated probe sites on Michigan electrodes, which possess a very convenient lay-out for manufacturing. ${ }^{[4,433,434]}$ Placement of the FET on the NE but not on the recording site allowed the researchers to combine the sensitivity of FETs and low-noise operations. Also, manufacturing of neural interface sites with an insulating $\mathrm{Si}_{3} \mathrm{~N}_{4}$ layer, although still in micrometer scale, for implantation in peroneal nerve trunk of a rat was reported in 1991. ${ }^{[435]}$ In 1996, the complementary metal oxide silicon (CMOS)-compatible technology was also applied for manufacturing of sophisticated on-chip circuitry for signal processing, ${ }^{[436]}$ while integration of FET and recording sites on NEs was reported in 2000. ${ }^{[437]}$

FET devices allow stimulation and recording on cultured neurons to be conducted in a non-invasive manner. All FETs have four terminals: the gate, drain, source, and substrate (Fig. 11A). The gate permits or blocks the flow of electrons by creating or eliminating a channel between the source and drain. Because a current is generated from electrons flowing from the source terminal toward the drain terminal in response to an applied voltage, the device allows the extracellular voltage of individual neurons attached to the gate terminal to be detected and measured. One of the earliest examples using an identified neuron of the leach attached to the non-metallized open gate of a FET demonstrated that the action potentials modulate directly with the source-drain current in silicon through capacitive coupling. ${ }^{[427]}$ Unlike previous attempts to measure potentials using electrodes, ${ }^{[438,439]}$ the FET device allows detection of signals below the threshold level and its response preserves the shape of the action potential. From this work, it was postulated that long-term, multi-site recording of the electrical activity of neurons at high resolution and high signal-to-noise ratios could be feasible by constructing patterns of FETs and with further engineering of the microelectronic circuitry.

The possibility for two-way, non-toxic communication between microelectronic FET chips and nerve cells was eventually realized using a hybrid circuit of a semiconductor chip with synaptically connected neurons from the snail (Fig. 11B). ${ }^{[440]}$ The siliconneuron-neuron-silicon circuit begins with a capacitive stimulator on the chip that excites a specific neuron. Signals are then transmitted in the neuronal network and elicit an action potential in the second neuron, whose postsynaptic excitation modulates the current of a FET on the chip. This achievement is a critical and fundamental step in neuroelectronic engineering and has immense significance in neuronal signal processing, neurocomputation, and neuroprosthetics.

It is no surprise that very few experiments have been carried out using mammalian cells in the development of neuroelectronic FET devices. ${ }^{[377,429,441,442]}$ Most of the work to this date has been conducted using individual or a small network of invertebrate nerve cells for several reasons. ${ }^{[427,432,440,443-445]}$ Most obviously, invertebrate nerve cells have large cell bodies to support efficient interfacing and allow convenient manipulation. This is especially true for works involving impaled electrodes. ${ }^{[427,432]}$ Working with smaller mammalian neurons and a larger network of cells requires progress in several key areas. $^{[440]}$ Stimulators with higher capacitance and transistors with lower noise are needed to stimulate and record from the smaller mammalian neurons. The ability to control neuronal circuit by guided growth (see Sections 5.2 and 5.3) with defined 

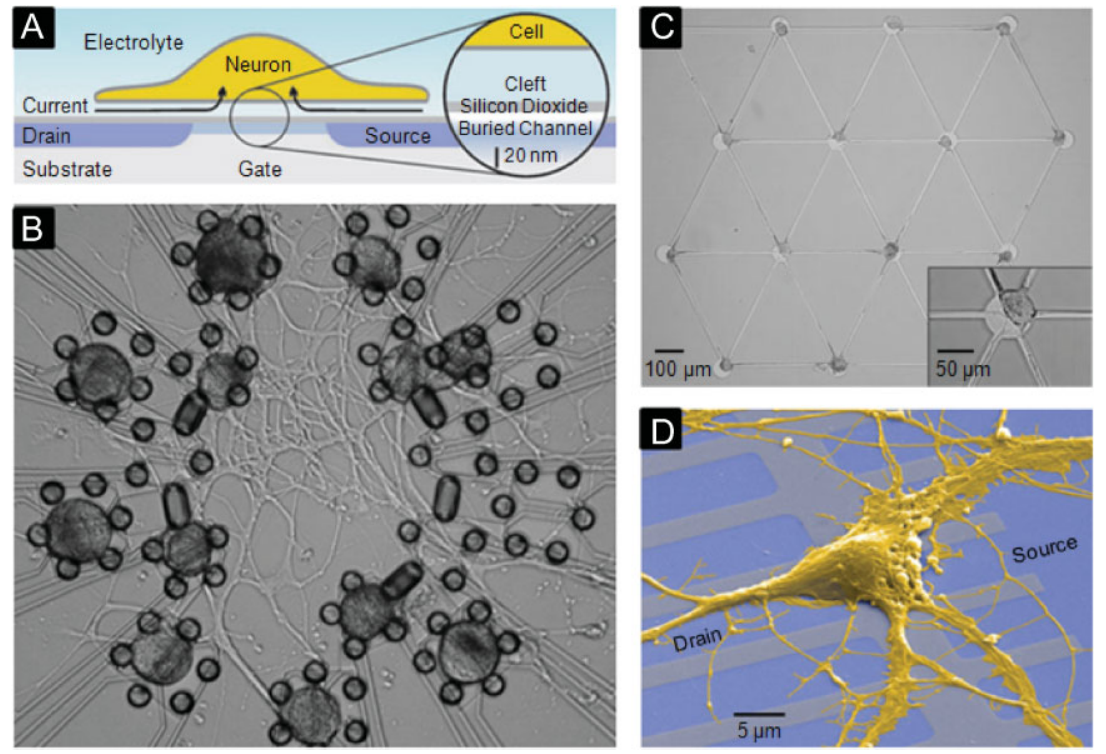

Figure 11. A) Schematic cross-section of a neuron on a buried-channel FET with blow-up (drawn to scale) of the contact area. During an action potential, current flows through the adhering cell membrane and along the resistance of the cleft between chip and cell. The resulting extracellular voltage in the cleft modulates the source-drain current. Adapted with permission from [449]. Copyright 2005, Wiley-VCH. B) Neuron silicon chip. Micrograph of neuronal cell bodies (dark bodies) in picket fences on a circle of two-way contacts connected by neurites (bright threads) after 2 days in culture. Reproduced with permission from [440]. Copyright 2001, National Academy of Sciences. C) Silicon chip with topographically controlled neuronal network on a $4 \times 4$ array of bi-directional electronic contact. An optical microscopy image after 2 days in culture. Details are illustrated in the magnified inset image. Adapted with permission from [445]. Copyright 2005, Wiley-VCH. D) Colorized electron micrograph of a hippocampal neuron on a silicon chip with linear array of $p$-type buried-channel transistors after 8 days in culture. Between source and drain leads are the open voltage-sensitive gates. The surface of the chip is chemically and structurally homogeneous, consisting of silica with a surface profile below $20 \mathrm{~nm}$. Adapted with permission from [449]. Copyright 2005, Wiley-VCH.

synaptic junctions and the application of very large scale integration (VLSI) technology are necessary to fabricate chips with a large number of contact sites for manipulation of larger neural networks.

Some of the most recent developments in planar neuroeletronic FET devices were made possible by advances in chip fabrication technology. Electrical imaging of neuronal activity of individual neurons and neuronal networks was mapped at a resolution of $7.8 \mu \mathrm{m}$ using a $1 \mathrm{~mm}^{2}$ array of 16384 sensor FETs fabricated by an extended CMOS technology. ${ }^{[46]}$ The large number and high density of recording sites made by VLSI technology allowed electrical imaging to be obtained noninvasively at a sub-cellular resolution without positioning and immobilizing individual neurons at specific locations of the substrate. This is a significant breakthrough since previous designs using electrolyte-oxide-semiconductor (EOS) FETs could only achieve a maximum resolution of $4 \mu \mathrm{m}$ in one dimension due to spatial constraints with routing the contact wires. The two-dimensional multi-transistor array was also applied to cultured brain slices to yield time-resolved images of electrical field potentials, demonstrating the ability of the new chip to interface large neuronal networks. ${ }^{[477]}$ Another key development is the establishment of a neuronal memory on a silicon chip by joining an excitatory chemical synapse between a pair of identified neurons with the silicon chip. ${ }^{[48]}$ With repetitive capacitor stimulation on the presynaptic cell, the strength of the soma-soma synapse can be enhanced and successfully recorded by a transistor located at the postsynaptic cell. This demonstration has significant implications for the development of biochips for chemical interfering with synaptic activity, as well as for integration of electronic circuits with neuronal activity.

The ability to control neurite outgrowth has also enabled the interfacing of a geometrically defined network of neurons to a semiconductor chip (Fig. 11C). ${ }^{[45]}$ By using topographical structures for cell immobilization, problems associated with guidance by chemical patterning such as insufficient adhesion and detachment of cell bodies (see Section 5.2.2) were circumvented. The combination of defined networks and extracellular interfacing made possible the supervision of network activity at the single-cell level. Finally, it was recently demonstrated that local transistor recording of individual mammalian neurons is feasible with EOS FETs that have a sufficiently low noise level (Fig. 11D). ${ }^{[42]}$ By taking advantage of the, so-called, buried channel design, which submerses the electron channel of the transistor a few nanometers from the interface in the silicon substrate (Fig. 11A), it was possible to reduce the low-frequency noise caused by electron tunneling between silicon and traps in the gate oxide. The overall design produced a signal-to-noise ratio that is sufficient for broadband recording of the small signals from mammalian neurons. This was unachievable with previous chip designs.

Neuronal interfacing via FET devices has the key advantage that this technique does not interfere with cell activity and affords long-term recording. By combining complementary microcircuit elements in chip design, it is possible to non-invasively interface individual neurons in both directions on a single chip. Recording at high resolution and high signal-to-noise ratios is possible but interfacing with mammalian cells will require additional progress in fabrication technology, material science, and biotechnology. The technology of neuroelectronics is likely to continue to benefit from the ever-expanding knowledge available in semiconductor technology, producing increasingly intricate and useful devices.

FETs, however, are not without disadvantages especially in comparison with Michigan and Utah electrodes. Overall, they do not provide the performance comparable to the currently used electrodes due to high noise. In general, simple metal recording/ stimulation sites connected to shielded FETs located at the base of an NE shank by an interconnect, should be able to give a better functioning device. The stability of cellular contact with FET is an important issue and is likely to prevent such devices from being used in in vivo experiments and in clinical settings. The intrinsic design of FET devices also plays a critical role as the devices are 
geared toward the study of neuronal signal processing and basic neuroscience research rather than the development of prosthetic devices. Experiments have seen inconsistent results due to variable strength of neuron-chip coupling and cell damage during the isolation process. Satisfactory recordings are highly dependent on a tight seal of the cell membrane and gate which is difficult to achieve at high consistency. Progress must be made in the development of better capacitors and transistors in order to enable interfacing with large networks of mammalian neurons at high resolution.

\subsection{Nanowire Field Effect Transistors for Neural Interface}

NW-FETs have been shown to be highly sensitive detectors of changes in local charge or electric field, ${ }^{[20,411,449-453]}$ an important property that can be utilized in NEs. The sensitivity and selectivity of chemically modified NW-FET detectors have been demonstrated for a variety of detection modalities including reversible binding and unbinding of virus particles (single-virus level) ${ }^{[411]}$ and proteins (as low as $100 \mathrm{fm}$ ). ${ }^{[42,453]}$ The latter figure represents a sensitivity limit $10^{4}-10^{9}$ times below that afforded by state-of-the-art ion sensitive planar FETs. ${ }^{[45-456]}$ Given the exquisite sensitivity of NW devices and the data provided in the section above on traditional FETs, ${ }^{[47-462]}$ it would be logical to test NW FETs for detection of neural activity. Significantly, NW FETs can be used for measurements from extremely small areas of neurons, for instance at or below the level of a single axon or dendrite. Moreover, the junction with the neuronal membrane can be tighter than in the case of planar FETs since the curvature of NWs allows them to protrude between the topological features of the cellular membrane. On a perfectly flat surface this gap is on the order of tens of nanometers, which is considered to be the result of the finite length of adhesion proteins, ${ }^{[455]}$ although it could possibly include other contributions as well. Decrease of this gap is possible when a nanoscale cylinder makes contact with the cell membrane as was recently demonstrated in the case of CNTs and a supported lipid bilayer membrane. ${ }^{[463]}$ In the specific case of cells, it is worth noting that the nanoscale topology of NWs may serve to promote adhesion; it was shown that nanostructured surfaces formed by CNT networks promote cellular adhesion and spreading, even in the absence of standard adhesion factors such as polylysine. ${ }^{[425,464]}$

Efficient design of hybrid nanoscale nanoelectronic/cellular interfaces requires a fabrication scheme that incorporates (i) NW device arrays with controllable structures and reproducible electronic properties, (ii) inert surface regions that prevent the adhesion of cells and formation of unwanted networks, and (iii) areas containing cell adhesion promoters that will mediate a tight junction between the cell and NW devices. Ultimately, the process of heterogeneous surface patterning must be compatible with already-fabricated NW devices.

NW device arrays suitable for NEs can be fabricated by aligning silicon NWs on the oxide surface of a silicon/silicon oxide substrate using either Langmuir-Blodgett or dry transfer techniques. ${ }^{[465,466]}$ Both methods yield highly aligned NWs with pitch that can be rationally varied between tens of microns to as small as tens of nanometers. A true advantage of this technique, which outlines a strength of bottom-up assembly in general, is that by multiple steps of partial passivation of the substrate (e.g., with photoresist) followed by NW deposition and removal of the passivation, it is possible to define regions with distinct NW materials on the substrate. Similar or distinct NWs, for example $p$-type and $n$-type materials, can thus be readily fabricated into functional device arrays using photo- or electron-beam lithography, followed by metallization and dielectric passivation of the metal regions. ${ }^{\text {[20] }}$ The device pitch is defined by NW spacing and the design of the lithography pattern; device arrays with pitches from more than $50 \mu \mathrm{m}$ to $400 \mathrm{~nm}$ and below can be fabricated using this approach.

In the case of these experiments, the surface of the NW devices was patterned with standard photolithography techniques using a combination of poly(lysine) and SAM-forming fluorinated silane as the cell adhesive and cell-repellent factor, respectively (see Sections 5.2.1 and 5.3). Using these bi-functional coatings, isolated neurons can be directed to extend neurites in a specific direction(s) crossing single NW devices (Fig. 12A). More complex patterns can also be created; examples include axons and dendrites crossing multiple NW devices that can serve as multiplexed inputs and outputs (Fig. 12C) and interconnected neural networks with neurites crossing an arrays of devices (Fig. 12D and E). ${ }^{[20]}$

Action potential signals can be measured in NW-FET electrodes interfaced with an axon. These measurements can also be benchmarked against IC potentials measured with a conventional glass microelectrode impaled in the soma. Direct temporal correlation between IC potentials and the signal recorded from the $p$-type NW was observed. Such behavior is expected since the potential outside the membrane becomes
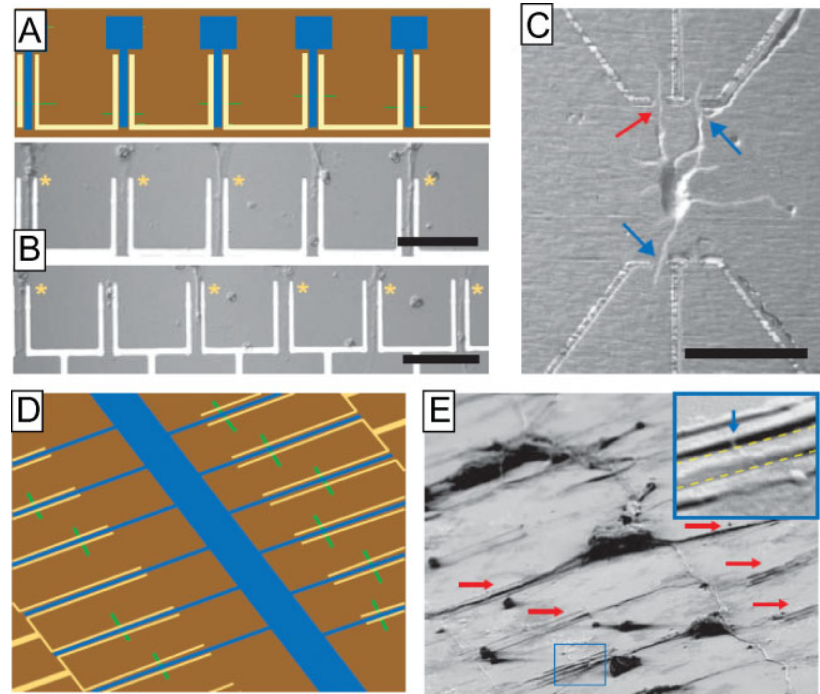

Figure 12. A) Schematic of single device/neuron patterning motif used for axon measurements. B) Arrays of axons with growth guided across an array of NW devices. Yellow asterisks denote locations where an axon was successfully guided between source and drain electrodes. Scale bars are $50 \mu \mathrm{m}$. C) A different neuron pattern where neurites are guided to form one $\mathrm{NW}$ /axon junction (red arrow) and two NW/dendrite junctions (blue arrows). Scale bar is $50 \mu \mathrm{m}$. D) Schematic of interconnected neuron motif. E) SEM image of fixed neurons exhibiting a neural network where soma are interconnected and neurites are interfaced with multiple NW devices (red arrows). (Inset) Zoom depicting an axon (yellow dotted lines) guided between source and drain electrodes and across a silicon NW (blue arrow). $B, C)$ Adapted with permission from [20]. Copyright 2006, AAAS. D,E) courtesy of Prof. C. Lieber, Harvard U. Boston, MA, USA. 
more negative, then more positive (opposite to the measured IC potential) and causes carrier accumulation (increased conductance) followed by depletion (decreased conductance), consistent with previously reported measurements from mammalian neurons interfaced with planar FETs. ${ }^{[42]}$ A variety of controls served to validate these measurements. ${ }^{[20]}$ First, it was observed that an increase in depolarizing current results in an increased frequency of signals measured by the IC electrode and NW FET. Secondly, an NW-axon junction failed to record signals after the axon was severed, although signals continued to be elicited in the soma. Finally, a set of electrodes with no NW recorded no signal from a growth-directed axon. Taken together, these controls demonstrate that extracellular recordings are a result of carrier modulation in the NW that is caused by changes at the local axon/ NW interface.

In addition to recording, stimulation of neurons at highly localized regions represents a useful technology since it could enable multiplexed nanoscale inputs that might be operated as "artificial synapses." The NW devices were used to apply biphasic excitatory pulse sequences to create detectible somatic action potential spikes measured by a microelectrode, a process that could be a result of capacitive coupling developed for traditional FETs $^{[17,460,467]}$ or highly local, reversible electroporation. ${ }^{[468]}$ The excitation shows a threshold of about $0.4 \mathrm{~V}$ and no potential spikes were recorded in the presence of tetrodotoxin, a common $\mathrm{Na}^{+}$ channel blocker that has been shown to completely inhibit action potentials as measured by multi-electrode arrays. ${ }^{[469]}$

Furthermore, NW-based electrodes make possible simultaneous measurement of axon/dendrite signal propagation, which represents a new level of integration that can be achieved with electronic devices. A device structure consisting of a linear array of 4-NW FETs, a gap, and 5-NW FETs was designed (Fig. 13) to investigate simultaneous and temporally resolved propagation and back propagation of action potential spikes in axons and dendrites, respectively. ${ }^{[20]}$ An optical image (Fig. 13) demonstrates well-defined growth of rat cortical neurons with the cell body localized in the gap and an axon and dendrite guided in opposite directions across the two linear NW FET arrays. The specific polarity of growth (e.g., axon across the 4 or 5-FET array) is not controlled, but is readily identified by the faster growing projection (the axon) during culture, and subsequently by electrical response and postmeasurement fluorescent imaging.

Multi-NW/neuron arrays were used to simultaneously detect conductance output from NWs following IC stimulation at the soma. ${ }^{[20]}$ Importantly, alternating $p$ - and $n$-type NW elements along a single axon can be integrated in one device. Following IC stimulation of action potential spikes in the soma, temporally correlated, alternating conductance peaks/dips in NW elements

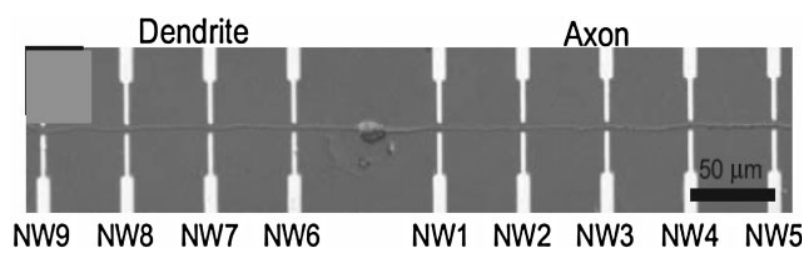

Figure 13. Optical image of a multi-NW/neuron arrays made from a cortex neuron with axon and dendrite aligned in opposite directions. Reproduced with permission from [20]. Copyright 2006, AAAS. were observed. These results are consistent with gating of the $p$ and $n$-type NWs by the change in membrane potential associated with the propagating action potential, and show that we can generate complementary signals in the hybrid structures. Stimulation of action potential spikes in the soma yields correlated conductance peaks in NW elements forming the $\mathrm{NW}$ /axon and NW/dendrite junctions. Qualitatively, these data demonstrate several key points. First, seven of the nine independently addressable NW/neurite junctions yield reproducible conductance spikes correlated with IC stimulation. This density of hybrid NW/neurite synapses on a single cell is unmatched by previous electrophysiological work. ${ }^{[442,460,469-472]}$ While previous studies using glass microelectrodes have recorded spike propagation in axons and dendrites, ${ }^{[470,471]}$ axon/dendrite propagation has not been measured simultaneously nor has the same level or recording points been achieved (although it has been demonstrated that measurements can be taken at multiple points by moving a single pipette probe). Second, the conductance spikes recorded along the axon by elements 1-5 maintain sharp peak shape and relatively constant peak amplitude. In contrast, the conductance spikes measured by elements 6-9 along the dendrite exhibit noticeable broadening and reduced amplitude.

Signals from the multiple, spatially separated NW/neurite junctions are recorded simultaneously, and thus enable spike propagation rates to be quantified in both axons and dendrites. ${ }^{[20]}$ Signal propagation rates of $0.16 \mathrm{~m} \mathrm{~s}^{-1}$ for dendrites and $0.43 \mathrm{~m} \mathrm{~s}^{-1}$ for axons were calculated. In trials with different neurons, Gaussian distributions of $0.15 \pm 0.04 \mathrm{~m} \mathrm{~s}^{-1}$ and $0.46 \pm 0.06$ for dendrites and axons respectively were found; these data are comparable to reported propagation rates measured by conventional electrophysiological methods. ${ }^{[473,474]}$

High-sensitivity, "multi-site" electrical recording of neuronal activity and signal propagation has similarities to optical methods, ${ }^{[475]}$ which rely on the injection of voltage-sensitive dyes, but also possesses advantages. For example, one or more of the NW/neurite junctions can be used as inputs to initiate and/or modulate signal propagation. As such, the NW device can be configured so that the middle NW junction can apply a variable potential input to an axon, while other NWs elements are used to record temporally resolved spike propagation following IC stimulation on the same axon. As the input on the control wire changes from 0 to $0.9 \mathrm{~V}$, the downstream propagation of the signal can be inhibited and ultimately blocked. These results suggest that inhibition is localized at a given NW/axon input, and is consistent with local anodic (hyper) polarization of the membrane at NW/axon synapses. This polarization inhibits and ultimately blocks the propagation of action potential spikes. ${ }^{[46-478]}$

These results show that the NW/neurite junctions can be used beyond simple recording and stimulation, and enable more subtle modulation of the spike propagation. It should be possible to extend this approach to modulation of dendritic signals at the level of individual dendrites. Continuous or analog control of the speed and amplitude of propagating signals through NW/ neurite junctions could enhance the understanding of synaptic processing in neuronal networks, especially considering that arrays of these artificial synapses could be used to modulate and simultaneously record signals with high spatial and temporal resolution. 
A complete discussion of NW FETs warrants consideration of unexplored areas and next steps, which will more clearly reveal potential advantages and disadvantages of NW-based neural interfaces. In the case of MEAs, ${ }^{[438,439]}$ planar FETs, ${ }^{[377,427,429,440-448]}$, and NW FETs, ${ }^{[20]}$ the magnitude of recorded signals is strongly dependent on cell-substrate junction quality. This factor may in fact be mitigated in the case of NW devices, since NWs protrude from the surface of the substrate ${ }^{[20]}$ and can therefore form an enhanced junction with the membrane. Although there is considerable evidence that micro- and nanoscale structures uniquely interact with membranes, ${ }^{[463,464]}$ a rational analysis of NW geometry, surface functionality, and pitch will be needed to determine the true nature of coupling in this particular system. Additionally, the substrate itself plays an important role in neuronal adhesion and viability, and is an advantage in the case of NW devices since NWs can be assembled on nearly any type of substrate, including flexible, biocompatible polymers. ${ }^{[465]} \mathrm{NW}$ devices fabricated on novel substrates could offer unique interfaces with soft, 3D tissue both in vitro and in vivo, and might moreover avoid the unwanted immune responses associated with conventional electrodes, ${ }^{[93-101]}$ but will need to be studied more carefully for cytotoxicity and biocompatibility in the context of both cells and tissues. Finally, at the present stage of development, the primary applicability of NW device arrays will be as tools for neuroscience on well-defined neurons and brain slice samples; integration of more complex systems such as prosthetics will require carefully-engineered arrays and surface chemistries.

\subsection{Photoactive Nanostructures for the Neuron Interface}

Optical stimulation of neurons is the foundation of vision. At the same time strong optical activity is very characteristic for nanostructures, and therefore lends itself to the design of optoelectronic interface with neural tissues. A recent review shows that technological capabilities of nanomaterials are promising for solving many challenges that researchers are facing in developing artificial retinas and similar devices. ${ }^{[479]}$ Activation of neurons via photostimulation is also relevant for standard NEs from the perspective of wireless operation of implantable devices using small hand-held sources of light. Despite the promise and importance, the actual amount of research activity in this area is not very high, probably as a result of the complexity of techniques and equipment necessary.

Most photoactive nanostructures studied so far can be described as semiconductor photoactive coatings with thicknesses in nanometer scale designed to function as artificial retinas. ${ }^{[479]}$ These coatings can be both organic ${ }^{[480]}$ or inorganic. ${ }^{[481-485]}$ The most popular organic material for optical stimulation of neurons is bacteriorhodopsin, while inorganic coatings are typically made from standard doped silicon or other semiconductors. By and large, the design of these devices is identical to solar cells. Similarly to other NEs, flexibility and mechanical compatibility with the retinal tissue was identified as one of the most important parameters of the long-term performance of the device ${ }^{[485]}$ in order to minimize the damage during ophthalmic surgery and to get better contact to eye neurons.
Macromolecules and complexes with nanoscale dimensions for optical stimulation of neurons were also utilized for optical stimulation of neurons. The purpose of these studies is typically unrelated to artificial vision; they are motivated primarily by the search of non-invasive methods for the temporally and spatially precise activation of neurons. Optical tools become very convenient for probing neuronal sensitivity, mimicking synaptic connections, elucidating patterns of neural connectivity, and unraveling neural circuits in complex neural networks. ${ }^{[486]}$ Starting from single neurons, one can genetically engineer ion channels in a neuron to have a photoactivated switch, which allows the researcher to turn them on and off using light. ${ }^{[487]}$ Optically gated ion channels can also be produced using a process dubbed "genetic chARGing" targeting the alpha subunit of the G protein. ${ }^{[488]}$ When such neurons are generated in the brain of a live organism, for instance drosophila flies, illumination of the neurons resulted in complex behaviors, such as jumping, wing beating, and flight, specific to the functionality of the brain area where the neuron modification was performed. ${ }^{[489]}$

Optical stimulation of individual neurons in brain slices in an arbitrary spatiotemporal pattern was achieved using two-photon uncaging of 4-methoxy-7-nitroindolinyl-glutamate which equates to the local photostimulated release of a neurotransmitter. The advantage of this technique is that it has single-cell and three-dimensional precision. By sequentially stimulating up to a thousand potential presynaptic neurons, detailed functional maps of inputs to a cell were generated. ${ }^{[490]}$ Similar purpose was behind the use of optical modulation of neuronal activity taking advantage of a light-driven chloride pump integrated in the cellular membrane. ${ }^{[41]}$ This system allows either knockout of single action potentials, or sustained blockade of spiking. When combined with calcium imaging, light-controlled chloride pumps produce a system for multimodal, high-speed, genetically targeted, all-optical interrogation of living neural circuits. ${ }^{[491]}$ Usefulness of optical control of neural activity can be demonstrated by the fact that light-induced activation allowed elucidation of the physiological model of depression and other malfunctions of CNS. ${ }^{[492-494]}$

Interesting possibilities are provided when semiconductor NP (quantum dots) are coupled to ion channels. These nanoscale colloids have a very high optical cross-section and can generate a large dipole moment around them. When conjugated to cell membrane in the vicinity of an ionic channel, NPs can potentially perturb the nerve membrane potentials by blocking ion transport through it. As a first step, toward this goal, an NP-neuron interface was made by attaching CdS NPs to neurons. ${ }^{[495]}$ After that, however multiple challenges were found on the realization of this goal starting from cytotoxicity of NPs (see Section 3.1) and non-specific binding to rapid endocytosis of the attached NPs inside the cell. ${ }^{[479]}$

One interesting example of quantum-dot based optically active interface is a hybrid bionanodevice made on the basis of HgTe NPs. These quantum dots absorb light and stimulate neural cells following a sequence of photochemical and charge-transfer reactions. ${ }^{[203]}$ HgTe NP films (Fig. 14A and B), were assembled by the LBL technique into a tightly packed (Fig. 14B) conductive composite film. Electrons and holes produced by excitons photogenerated in quantum-confined HgTe resulted in cathodic photocurrent with transients characterized by the strong initial 

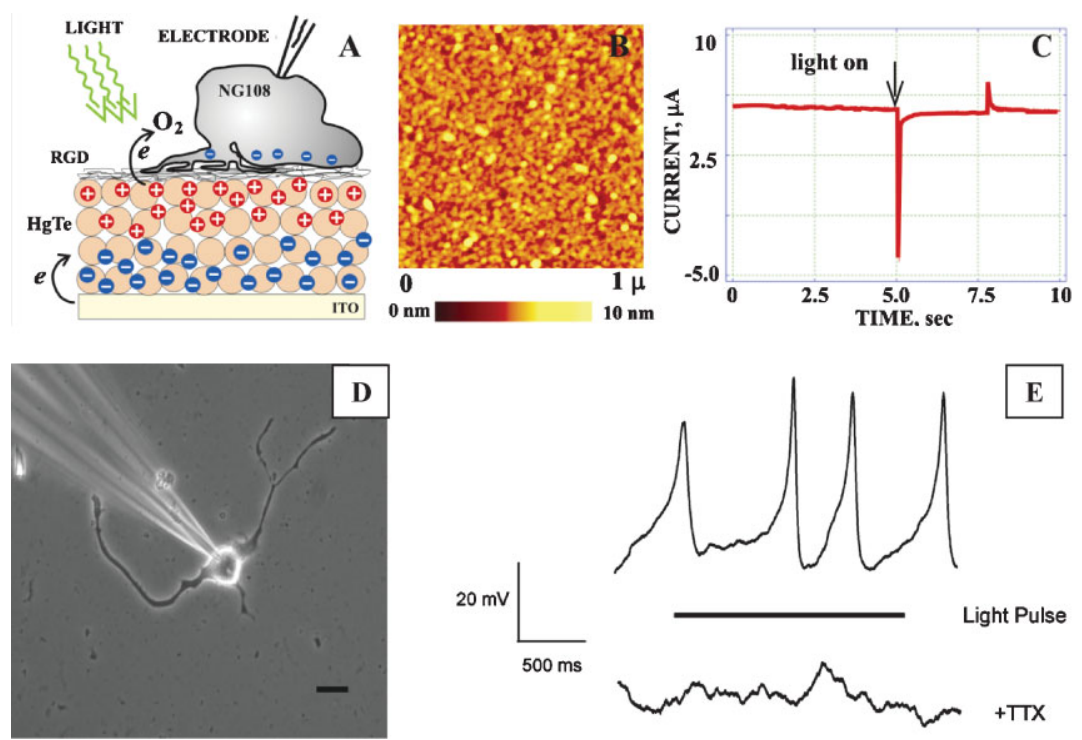

Figure 14. A) Schematics of the photoinduced stimulation of neurons by the layer of NPs. B) Atomic force microscopy image of the NP layers before adhesion of neurons. C) Kinetics of the photoinduced current in the NP layer. D) Optical microscopy of the patch clamp contacting the neuron under investigation. E) Train of the transmembrane potential signals of neuron adhering to the NP film excited by the train of light pulses. Lower curve: the same signals after addition of tetrodoxine (TTX). Adapted with permission from [203]. Copyright 2007, ACS.

spike (Fig. 14C). The photocurrent originates from the photoinduced electron transfer from NPs to molecules of oxygen present in solution. The positive charge at the outer interface of the film is accumulated due to fairly slow electron transport through NP solids, which is particularly true for LBL films. ${ }^{[496]}$ This charge is used to excite differentiated NG108-15 neuron cells adherent to the NP film, which can be registered with IC trans-membrane potential measurements with patch clamp (Fig. 14D). When stimulated with a $532 \mathrm{~nm}$ laser, neurons show depolarization. The LBL method offers the possibility to engineer the interface and fine-tune neural response in nano-bio devices. As such, additional LBL films of clay sheets were deposited on top of the NP films. They imparted greater biocompatibility and reduced gap between the surface of the device and neural membrane. Also, clay-polyelectrolyte films increased the dielectric constant and ionic conductivity. ${ }^{[497,498]}$ As a result, depolarization of the cells greatly increased (Fig. 14E) and many of them demonstrated action potential when depolarization generated a signal in another neuron. Overall the mechanism of transduction of optical signal in the neural response can be described as induction of a large voltage drop across neuronal membrane due to photocurrents in the NP layers. In response to that, $\mathrm{Na}^{+}$ion channels open and the action potential is generated. The mechanism of the photoinduced stimulation was confirmed using a potent neurotoxin tetrodoxin, which functions as $\mathrm{Na}^{+}$ion channel blocker and dampened the cellular response (Fig. 14E) to light.

\section{Future Perspectives}

There is an urgent need for finding therapies to neural disorders with strategies involving drug delivery, tissue repair, and electrical implants. The latter will also greatly benefit many other diseases treatable by appropriate electrical stimulation of CNS or peripheral nerves. These advancements rely on better diagnostics, therapeutic targets, and ex vivo modeling of neural tissue as tools for guiding these strategies. As one can see neural interfaces represent a very diverse field of practical applications with challenging requirements, which are only partially fulfilled by current methods and materials. In our opinion, the future of neural interfaces lies in the integration of traditional approaches used so far and nanoscale materials and devices that can enhance, improve and, in some cases, revolutionize the current technologies used to investigate, access, monitor, and stimulate neural tissues. In this part, we will present a brief outline of the most fruitful and important venues of nanotechnology development in the next 5-10 years that can address the needs of neurobiology. In doing so, we do not intend to predict the future, but rather to answer the question "Where one might go from here?" given the state-of-the-art described in all the sections above.

Since the technology of the NEs is fairly well developed, new types of coatings that reduce inflammation and improve survival of neurons in the neural tissue will probably be the first applications of nanotechnology in this field. Nanoscale topological features have been shown to increase cell adhesion and viability (see Sections 5.1-5.5), ${ }^{[362]}$ which may be exploited to make neuron-device coupling more reliable. Novel substrate coatings offered by nanomaterials can be used to immobilize cells and increase the number of cells growing neurites. One very successful example is peptide nanofibers, which enable rapid selective neuronal differentiation by amplification of bioactive epitopes presented to cells. ${ }^{[257]}$ This can certainly be extended to many NEs. Incorporation of anti-inflammatory $\operatorname{drugs}^{[383,388,499,500]}$ in the coatings and neuronal guidance toward the electrode by self-assembled scaffolds represent the directions with greatest immediate and practical importance. The approaches described in Sections 4.1, 5.2, and 5.4 will make possible substantial improvement in the performance of NEs. The combination of neural guidance and drug elution capabilities in one coating should be strongly considered.

Future development of nanostructured coatings will also target significant increase of charge injection capacity and reduction of interface impedance. This task might be achieved by incorporation of nanoscale features in traditional $\mathrm{Ir} / \mathrm{IrO} \mathrm{I}_{x}$ electrodes or coating metals with other nanomaterials such as CNTs. This venue is critical for the further miniaturization of the traditional stimulation electrodes necessary for more selective, "intelligent" excitation of neuronal cells and for the reduction of inflammation effects by using smaller and more compliant NEs. Drastically increased improvement of electrical and biological characteristics of the interface between neuronal cells and NE materials is a prerequisite for the success of MEAs (see Sections 5.1.2 and 2.3.3). It is also much needed for further reduction of electrode 
dimensions in traditional Michigan electrodes necessary for reduction of scarring of neural tissue.

Considering the NE challenges outlined in Section 2.3, it might also be the time to completely rethink the design of an electrode and to change the existing status quo in NEs. Ideally, a neural stimulation electrode should be integrated in the tissue as a compliant flexible material resulting in minimal disturbance to the tissue. For cuff implants, electrodes must be well integrated with the thin insulating backing to prevent microscale delamination of the metal coating. ${ }^{[92]}$ Nanoscale topology and/or coating with high aspect ratio features on the surface are known to improve charge injection in neurons, ${ }^{[501-505]}$ and can certainly be added to it. Considering brain and spinal stimulation, the electrodes should also be of limited diameter because larger shaft implants have been shown to activate immune responses in greater extent. ${ }^{[76,111]}$ Hypothetically speaking, the electrode should also provide side branches in the form of flexible soft mesoscale leaflets to reach larger number of cells or other means to interpenetrate the tissue much better. The leaflet or similar geometry of the side branches as opposed to more common fibrous geometry will generate area of contact with the neuronal cells sufficient for activation. The branches should extend probably $75-100 \mu \mathrm{m}$ off the main insulated shaft to reach the greater number of neurons and penetrate through a potential encapsulation sheath. An NE design incorporating some of these design ideas not necessarily to the letter but rather mimicking the structure of neural tissue itself with, for instance, in situ gellation of conductive SWNT composite as a likely implementation of this approach, will make excitation process more efficient and less traumatic for the tissue. However, it does imply difficulties with implantation process, which may require further changes in the concept of the electrode, while retaining the general functionalities and design purposes of the new NE as described above. The questions about control of the quality of interface and its reproducibility need to be addressed as well, which will likely require potentially integration of membrane potential contrast agents in the electrode structure as well.

Manufacturing of this or similar devices, although technically possible, is limited by the physical properties of the available materials. Electrical and mechanical properties represent the key limitations, and should a highly conductive yet mechanically strong and flexible material becomes available, a new generation of NEs can be designed and tested. Needless to say, such materials will also be valuable for other applications in biomedical engineering and health technologies. Therefore, fundamental studies on the development of strong, easily manufacturable, highly conductive materials that can resolve issues with mechanical properties of NE (see Section 2.3.2) would be quite important. The development of such materials can go along several directions, which are likely to include both conductive polymers, ${ }^{[506]}$ CNT composites, ${ }^{[19,152,200,507]}$ and potentially other previously unused materials. In this respect, the studies of the chronic long-term toxicity of such materials over the period of implantation spanning several years are also very necessary. They will probably become a bottleneck because they cannot start until such material and method of manufacturing of electrodes are identified. As we saw in Section 3.2, the tissue effect is strongly dependent on method of manufacturing of such composites and not just on the individual ingredients.
FETs (Section 5.7) as recoding sites do share many challenges of the common shank and cuff electrodes but do provide an interesting direction of NE especially if better connectivity with neuron membrane can be obtained. High sensitivity and gentler capacitive coupling are the key advantages of this technology. However, studies on FETs were carried out only in vitro and, hence, animal studies and design of the chip suitable for such studies would be most necessary. One of the greatest challenges here is the maintenance of the small gap between the device and target cell because the device performance is critically dependent on it. Coatings and nanostructured surfaces can significantly contribute toward defeating this challenge. Also, a "soft" nanoscale FET that can reside on the cell surface will integrate very well with the outline of the new generation of $\mathrm{NE}$ described above. Therefore, highly flexible FETs compliant with neural tissues should be developed. Without this step, their utility will be limited due to inflammatory reactions. The flexible FETs are likely to need new semiconducting and insulating media because traditional $\mathrm{Si} / \mathrm{SiO}_{2}$ chips are fairly hard and brittle.

The new generation of FETs from NWs (Section 5.8) may be used to dramatically increase the resolution of detection and stimulation of neuronal activity. ${ }^{[20]}$ One of the advantages of NW FETs is the ability to readily integrate distinct materials, such as $n$ and $p$-type NWs, and hence, low-power logic gates, followers, and amplifiers, that are ubiquitous in microelectronics, and have been interfaced with whole cells as planar FETs. ${ }^{[462]}$ This opens up future opportunities for on-NE processing elements, new means of interfacing, ${ }^{[58,509]}$ and recording/excitation of cellular signals from multiple sites. ${ }^{[510,511]}$ This function will also be helpful for establishing the mechanisms of therapeutic actions of DBS, ${ }^{[512]}$ which are currently quite obscure (see Section 2.3.5) and represents one of the important challenges of the field.

Classical planar and NW FETs share some of these exciting opportunities, and in particular could satisfy one of the acute needs of basic research. Configuring FETs to behave as chemical sensors could enable one to correlate neurotransmitter release to electronic signaling for NW ${ }^{[513,514]}$ and planar FETs ${ }^{[17,515-517]}$ with individual nanodevices in an array being designed, through specific chemical interactions or modulation of applied redox potentials, to simultaneously detect a matrix of biologically significant species. Special attention here should probably be given to FETs from CNTs using special properties of NT composites $^{[16,518]}$ and/or taking advantage of new types of electronic devices possible from these nanoscale building blocks such as ballistic FETs ${ }^{[519]}$ or ultrasensitive capacitors. ${ }^{[520]}$ FETs from CNTs have an excellent track record, but have never been used in neural applications..$^{[521-523]}$

More NEs should be made incorporating light-sensitive materials for light-addressable interfaces, ${ }^{[203]}$ which is likely to be one of the most promising directions for the future development of light-sensitive interfaces. Note though, that mechanical properties and miniaturization of active electrode surfaces acquire probably even greater importance when photoactive NEs are designed to serve as an artificial retina. The presence of photoreactions at the interface gives additional restrictions on the materials to be used, their biocompatibility, and long-term stability. As such, many dyes adsorbing in visible light including previously used bacteriorhodopsin are also subject to slow photooxidation, and thus cannot persist in the implant for 
a long time. More stable inorganic semiconductor materials that are active in the visible spectrum tend to contain heavy metals, which are likely to cause problems with long-term biocompatibility. Hence finding and testing alternate nanostructured materials and potentially differently doped inorganic nanocolloids should be charted as one of the future tasks in this area.

As well, photoreactions often generate free radicals including ROS, which are found to initiate neural damage. They certainly need to be minimized and mitigated. Fullerene coatings (see Section 4.1.2) can serve as an intermediate solution for this offering both strong photovoltaic activity in the visible range and radical savaging. CNTs may also give adequate performance and can possibly surpass other materials as a potential candidate for an artificial retina. However, their adsorption spectrum tends to include IR bands and thus may result in greater sensitivity to IR emission, which may not be a disadvantage. Besides artificial retinas, this property could also be used for remote electrodeless excitation of implanted NE. Human tissues have a window of transparency in near IR. In perspective, it is possible to design a photosensitive cuff electrode that can be positioned around a nerve and stimulated by a small red laser similar to classroom pointer.

Nanoscale materials for neural interfaces are also associated with systemic drug delivery and imaging using organic and inorganic NPs (see Sections 4.1.1 and 4.2). These tasks are particularly significant for the diagnostics and treatment of brain cancers and malfunctions of other parts of CNS. Although much (see Section 4.2) work had been done, this is still a challenging problem. Detailed understanding of the regularities and tendencies that govern the permeation of NPs through the $\mathrm{BBB}$ in respect to size, charge, and surface functionalities and subsequent maximization of this function is one of the important future targets in this area. The addition of therapeutic functions, for instance drug delivery, and imaging capabilities on top of BBB transparency will lead to a new generation of treatment methods for brain tumors. This will be particularly advantageous for early stages of tumors when vasculature has not developed the characteristic leaks and become more permeable. Although magnetic NPs have been FDA approved and used since 1980s, detailed understanding of their transport to neural tissues and the degree of its external positioning control in humans is still needed.

More generally, we believe that nanotechnology can also strongly impact in vitro drug assays ${ }^{[66]}$ necessary for the treatment of neurodegenerative disorders, neural restoration, inflammations in CNS and peripheral nervous system, and psychological deceases. As an example, the scalable nature and biocompatibility of FET, especially NW FETs, devices lends them to new methods of drug selection with much increased rate and accuracy of screening. One of the interesting challenges in this area is switching from the traditional screening in $2 \mathrm{D}$ plates to $3 \mathrm{D}$ scaffolds since neurons have extensive volumetric interconnections critically relevant to their performance that are distorted in 2D systems. Finding substrates for 3D drug screening will likely require the combination of both nanoscale and microscale features of the material. ${ }^{[524,525]}$

Last but not least, we also would like to address the use of stem cells in combination with the materials discussed above. The discovery of NSC in adult brains has a crucial significance, in our opinion, for neural interfaces. Yet very little work is being done in this area and only very recently. ${ }^{[387]}$ NSCs have the ability to transform into functional neurons ${ }^{[526,527]}$ and integration of NSCs with the electrode can simplify its implantation, increase the number of neurons in actual contact with the NE, and create a living interface with the surrounding tissue. It is envisioned that NSCs will differentiate once implanted, develop processes penetrating through the encapsulating layer, and form natural biological connections to the surrounding neurons. The fact that NSCs can create functional signal-bearing networks similar to normal neurons has already been demonstrated. ${ }^{[528]}$ Importantly, NSCs can also be developed from hematopoietic CD34-positive stem cells from peripheral blood of adults, ${ }^{[529-533]}$ therefore, making possible the development of the interface with the patient's own stem cells. This will greatly reduce or possibly completely eliminate the immune response of the nervous tissue and can resolve many challenges outlined above.

\section{Acknowledgements}

Thanks are due to Prof. Andreas Offenhauzer, Prof. Adam Curtis, Dr. Allison Beattie, Prof. Matis Riehle, and Prof. Chris Wilkinson for useful textual contribution to the review, which were partially incorporated in the manuscript in Section 5.2.2. Stimulating discussions, clarifications, and insights of Profs. Ken Wise, Khalil Najafi, Daryl Kipke, David Martin were also greatly appreciated.

Received: July 13, 2008

Revised: January 1, 2009

Published online: July 8, 2009

[1] D. F. Emerich, C. Halberstadt, C. Thanos, J. Biomed. Nanotechnol. 2007, 3, 235.

[2] K. K. Jain, Clin. Chem. 2007, 53, 2002.

[3] K. K. Jain, Neurodegener. Dis. 2007, 4, 287.

[4] R. S. Kane, A. D. Stroock, Biotechnol. Prog. 2007, 23, 316.

[5] K. Najafi, Handbook of Microlithography, Micromachining, and Microfabrication, SPIE-International Society for Optical Engine, Chicago 1997, p. 517.

[6] D. Banks, Eng. Sci. Educ. J. 1998, 7, 135.

[7] P. Heiduschka, S. Thanos, Prog. Neurobiol. 1998, 55, 433.

[8] T. Stieglitz, J.-U. Meyer, in Microsystem Technology in Chemistry and Life Science (Eds: A. Manz, H. Becker), Springer, Berlin 1998, 131.

[9] J. P. Donoghue, Nat. Neurosci. 2002, 5, 1085.

[10] K. D. Wise, IEEE Eng. Med. Biol. Magazine 2005, 24, 22.

[11] T. M. Pearce, J. C. Williams, Lab Chip 2007, 7, 30.

[12] G. A. Silva, Nat. Rev. Neurosci. 2006, 7, 65.

[13] J. M. Koziara, P. R. Lockman, D. D. Allen, R. Mumper, J. Pharm. Res. 2003, 20, 1772.

[14] P. Yao, J. Huang, C. Kang, P. Pu, J. Chang, Zhongguo Yixue Kexuepuan Xuebao 2006, 28, 481.

[15] A. Bianco, K. Kostarelos, C. D. Partidos, M. Prato, Chem. Comm. 2005, 571.

[16] M. K. Gheith, T. C. Pappas, A. V. Liopo, V. A. Sinani, B. S. Shim, M. Motamedi, J. P. Wicksted, N. A. Kotov, Adv. Mater. 2006, 18, 2975.

[17] L. Wang, D. Fine, D. Sharma, L. Torsi, A. Dodabalapur, Anal. Bioanal. Chem. 2006, 384, 310.

[18] T. J. Webster, M. C. Waid, J. L. McKenzie, R. L. Price, J. U. Ejiofor, Nanotechnology 2004, 15, 48. 
[19] M. K. Gheith, V. A. Sinani, J. P. Wicksted, R. L. Matts, N. A. Kotov, Adv. Mater. 2005, 17, 2663

[20] F. Patolsky, B. P. Timko, G. Yu, Y. Fang, A. B. Greytak, G. Zheng, C. M. Lieber, Science 2006, 313, 1100.

[21] G. Ruan, A. Agrawal, A. M. Smith, X. Gao, S. Nie, Rev. Fluoresc. 2006, 3, 181.

[22] M. R. Warnement, I. D. Tomlinson, S. J. Rosenthal, Curr. Nanosci. 2007, 3, 273.

[23] K. T. Thurn, E. M. B. Brown, A. Wu, S. Vogt, B. Lai, J. Maser, T. Paunesku, G. E. Woloschak, Nanoscale Res. Lett. 2007, 2, 430

[24] K. Khosravi-Darani, A. Pardakhty, H. Honarpisheh, V. S. N. M. Rao, M. R. Mozafari, Micron 2007, 38, 804.

[25] W. Cai, A. R. Hsu, Z.-B. Li, X. Chen, Nanoscale Res. Lett. 2007, 2, 265.

[26] H. M. E. Azzazy, M. M. H. Mansour, S. C. Kazmierczak, Clin. Biochem. 2007, 40, 917.

[27] W. Jiang, A. Singhal, H. Fischer, S. Mardyani, W. C. W. Chan, BioMEMS Biomed. Nanotechnol. 2006, 3, 137.

[28] I. L. Medintz, H. T. Uyeda, E. R. Goldman, H. Mattoussi, Nat. Mater. 2005, 4, 435 .

[29] J. L. West, N. J. Halas, Annu. Rev. Biomed. Eng. 2003, 5, 285.

[30] J. E. Jensen, R. R. Conn, G. Hazelrigg, J. E. Hewett, Am. J. Sports Med. $1985,13,27$.

[31] A. H. Gordon, C. W. Schwabe, The Quick and the Dead: Biomedical Theory in Ancient Egypt, Brill Academic Publishers, Leiden, The Netherlands 2004.

[32] P. L. Gildenberg, Pain Med. 2006, 7, S7.

[33] W. L. C. Rutten, Annu. Rev. Biomed. Eng. 2002, 4, 407.

[34] R. A. Normann, Nat. Clin. Pract. Neuro. 2007, 3, 444.

[35] L. Galvani, De Bononiensi Scientarium et Artium Instituto adque Academia Commentarii $1791(7), 363$.

[36] M. Pruel, in: A History of Neurosurgery (Eds: S, Greenblatt, T, Dagitf, M. Epstein ), American Association of Neurological Surgeons, Park Ridge, IL 1997, p. 99.

[37] W. Isaacson, Benjamin Franklin: An American Life, Simon \& Schuster, New York 2003.

[38] B. Franklin, Phil. Trans. Roy. Soc. 1758(50), 481.

[39] A. L. Hodgkin, A. F. Huxley, Nature 1939, 144, 710.

[40] C. A. Terzuolo, T. Araki, Ann. N. Y. Acad. Sci. 1963, 94, 547.

[41] M. Verseano, K. Negishi, J. Gen. Physiol. 1960, 43, 177.

[42] F. A. Spelman, IEEE Eng. Med. Biol. Magazine 1999, 18, 27.

[43] K. D. Wise, J. B. Angell, A. Starr, IEEE Trans. Biomed. Eng. 1970, BME-17, 238.

[44] K. D. Wise, J. B. Angell, IEEE Trans. Biomed. Eng. 1975, BME-22, 212.

[45] K. Jones, P. Campbell, R. Normann, Ann. Biomed. Eng. 1992, 20, 423.

[46] K. Bradley, Pain Med. 2006, 7, S27.

[47] D. Harnack, C. Winter, W. Meissner, T. Reum, A. Kupsch, R. Morgenstern, J. Neurosci. Meth. 2004, 138, 207.

[48] J. M. Kerns, A. J. Fakhouri, H. P. Weinrib, J. A. Freeman, Neuroscience 1991, 40, 93

[49] D. R. Merrill, M. Bikson, J. G. R. Jefferys, J. Neurosci. Meth. 2005, 141, 171.

[50] A. L. Benabid, Curr. Opin. Neurobiol. 2003, 13, 696.

[51] M. A. Lebedev, M. A. L. Nicolelis, Trends Neurosci. 2006, 29, 536.

[52] R. R. Llinas, K. D. Walton, M. Nakao, I. Hunter, P. A. Anquetil, J. Nanoparticle Res. 2005, 7, 111

[53] F. A. Mussa-Ivaldi, L. E. Miller, Trends Neurosci. 2003, 26, 329.

[54] F. A. Spelman, Audiol. Neurootol. 2006, 11, 77.

[55] a) P. L. Marzella, G. M. Clark, Acta Otolaryngol. 1999, 119, 407; b) X. Navarro, T. B. Krueger, N. Lago, S. Micera, T. Stieglitz, P. Dario, J. Periph. Nerv. Syst. 2005, 10, 229

[56] G. Schalk, J. Neural Eng. 2008, 5, P1.

[57] C. Haberler, F. Alesch, P. R. Mazal, P. Pilz, K. Jellinger, M. M. Pinter, J. A. Hainfellner, H. Budka, Ann. Neurol. 2000, 48, 372.

[58] J. L. Vitek, T. Hashimoto, J. Peoples, M. R. DeLong, R. A. E. Bakay, Movement Disord. 2004, 19, 907.
[59] J.-Y. Chang, J. Pharmacol. Exp. Ther. 2004, 309, 1.

[60] C. Hamani, M. P. McAndrews, M. Cohn, M. Oh, D. Zumsteg, C. M. Shapiro, R. A. Wennberg, A. M. Lozano, Ann. Neurol. 2008, 63, 119.

[61] M. W. Keith, Microsurgery 2001, 21, 256.

[62] K. E. Matzel, U. Stadelmaier, M. Hohenfellner, W. Hohenberger, Dis. Colon Rectum 2001, 44, 59.

[63] J. Rozman, B. Zorko, M. Bunc, M. Zitko, Artif. Organs 2002, 26, 241.

[64] J. Andrews Russell, Ann. N. Y. Acad. Sci. 2003, 993, 1.

[65] P. J. Goodnick, A. J. Rush, M. S. George, L. B. Marangell, H. A. Sackeim, Expert Opin. Pharmacother. 2001, 2, 1061.

[66] E. H. Paul, III, B. N. Charles, Expert Opin. Pharmacother. 2006, 7, 2323.

[67] T. Matsumoto, T. Kanno, Peptides 1984, 5, 285.

[68] O. E. Osadchii, V. M. Pokrovskii, O. G. Kompaniets, A. N. Kurzanov, Bull. Exp. Biol. Med. 1997, 123, 427.

[69] M. Yamazaki, T. Sakaguchi, Brain Res. 1989, 484, 357.

[70] A. Yoshizaki, Y. Takagi, T. Kanno, Biomed. Res. 1992, 13, 303.

[71] N. I. Ziyatdinova, A. A. Gainullin, A. U. Ziganshin, T. L. Zefirov, Bull. Exp. Biol. Med. 2004, 137, 425.

[72] T. Stieglitz, J.-U. Meyer, Microsystems 2007, 16, 41.

[73] J. Gimsa, B. Habel, U. Schreiber, U. V. Rienen, U. Strauss, U. Gimsa, J. Neurosci. Meth. 2005, 142, 251.

[74] D. B. McCreery, W. F. Agnew, T. G. H. Yuen, L. Bullara, IEEE Trans. Biomed. Eng. 1990, 37, 996.

[75] R. D. Foreman, B. Linderoth, J. L. Ardell, K. W. Barron, M. J. Chandler, S. S. Hull, G. J. TerHorst, M. J. L. Dejongste, J. A. Armour, Cardiovasc. Res. 2000, 47, 367

[76] L. C. R. Wim, Annu. Rev. Biomed. Eng. 2002, 4, 407.

[77] B. Coburn, Crit. Rev. Biomed. Eng. 1989, 17, 133

[78] F. Rattay, Neuroscience 1999, 89, 335.

[79] C. C. McIntyre, M. Savasta, L. K.-L. Goff, J. L. Vitek, Clin. Neurophysiol. 2004, 115, 1239

[80] E. J. Tehovnik, A. S. Tolias, F. Sultan, W. M. Slocum, N. K. Logothetis, J. Neurophysiol. 2006, 96, 512 .

[81] S. R. F. Rattay, P. Lutter, K. Minassian, B. Jilge, M. R. Dimitrijevic, Neuromodulation 2003, 6, 42.

[82] B. Wessling, A. Besmehn, W. Mokwa, U. Schnakenberg, J. Electrochem. Soc. 2007, 154, F83

[83] E. Slavcheva, R. Vitushinsky, W. Mokwa, U. Schnakenberg, J. Electrochem. Soc. 2004, 151, E226.

[84] I.-S. Lee, C.-N. Whang, J.-C. Park, D.-H. Lee, W.-S. Seo, Biomaterials 2003, 24, 2225.

[85] T. M. Silva, J. Rito, M. G. S. Ferreira, I. Fonseca, K. Watkins, J. Mater. Sci.: Mater. Med. 1996, 7, 261.

[86] S. F. Cogan, A. A. Guzelian, W. F. Agnew, T. G. H. Yuen, D. B. McCreery, J. Neurosci. Meth. 2004, 137, 141.

[87] M. HajjHassan, V. Chodavarapu, S. Musallam, Sensors 2008, 8, 6704.

[88] P. J. Rousche, D. S. Pellinen, D. P. Pivin, Jr, J. C. Williams, R. J. Vetter, D. R. Kipke, IEEE Trans. Biomed. Eng. 2001, 48, 361.

[89] A. J. Engler, S. Sen, H. L. Sweeney, D. E. Discher, Cell. 2006, 126, 677.

[90] D. E. Discher, P. Janmey, Y.I. Wang, Science 2005, 310, 1139.

[91] H. Specht, F. Krueger, H. J. Wachter, O. Keitel, C. Leitold, M. Frericks, Med. Device Mater. III, Proc. 3rd Mater. Processes Med. Devices Conf. 2006, p. 169.

[92] U. M. Twardoch, J. Appl. Electrochem. 1994, 24, 835.

[93] J. N. Turner, W. Shain, D. H. Szarowski, M. Andersen, S. Martins, M. Isaacson, H. Craighead, Exp. Neurol. 1999, 156, 33.

[94] D. H. Szarowski, M. D. Andersen, S. Retterer, A. J. Spence, M. Isaacson, H. G. Craighead, J. N. Turner, W. Shain, Brain Res. 2003, 983, 23.

[95] Y.-T. Kim, R. W. Hitchcock, M. J. Bridge, P. A. Tresco, Biomaterials 2004, $25,2229$.

[96] R. Biran, D. C. Martin, P. A. Tresco, Exp. Neurol. 2005, 195, 115.

[97] D. J. Edell, V. V. Toi, V. M. McNeil, L. D. Clark, IEEE Trans. Biomed. Eng. 1992, 39, 635. 
[98] S. J. A. Davies, M. T. Fitch, S. P. Memberg, A. K. Hall, G. Raisman, J. Silver, Nature 1997, 390, 680.

[99] R. W. Griffith, D. R. Humphrey, Neurosci. Lett. 2006, 406, 81.

[100] R. Griffith, J. Soria, J. G. Wood, Exp. Neurol. 2000, 161, 297.

[101] J. W. Fawcett, R. A. Asher, Brain Res. Bull. 1999, 49, 377.

[102] A. M. P. Turner, N. Dowell, S. W. P. Turner, L. Kam, M. Isaacson, J. N. Turner, H. G. Craighead, W. Shain, J. Biomed. Mater. Res. 2000, 51, 430.

[103] P. M. St, John, L. Kam, S. W. Turner, H. G. Craighead, M. Issacson, J. N. Turner, W. Shain, J. Neurosci. Meth. 1997, 75, 171.

[104] J. Subbaroyan, C. Martin David, R. Kipke Daryl, J. Neural Eng. 2005, 2, 103.

[105] H. Lee, R. V. Bellamkonda, W. Sun, M. E. Levenston, J. Neural Eng. 2005, $2,81$.

[106] A. Gilletti, J. Muthuswamy, J. Neural Eng. 2006, 3, 189.

[107] G. C. McConnell, T. M. Schneider, J. Owens, R. V. Bellamkonda, IEEE Trans. Biomed. Eng. 2007, 54, 1.

[108] R. Biran, D. C. Martin, P. A. Tresco, J. Biomed. Mater. Res, Part A 2007, 82A, 169.

[109] Y. T. Kim, R. W. Hitchcock, M. J. Bridge, P. A. Tresco, Biomaterials 2004, 25, 2229.

[110] E. A. Nunamaker, E. K. Purcell, D. R. Kipke, J. Biomed. Mater. Res, Part A 2007, 83A, 1128.

[111] J. P. Seymour, D. R. Kipke, Biomaterials 2007, 28, 3594

[112] F. Keohan, X. F. Wei, A. Wongsarnpigoon, E. Lazaro, J. E. Darga, W. M. Grill, J. Biomater. Sci, Polym. Ed. 2007, 18, 1057.

[113] C. S. Bjornsson, S. J. Oh, Y. A. Al-Kofahi, Y. J. Lim, K. L. Smith, J. N. Turner S. De, B. Roysam, W. Shain, S. J. Kim, J. Neural Eng. 2006, 3, 196.

[114] V. S. Polikov, P. A. Tresco, W. M. Reichert, J. Neurosci. Meth. 2005, 148, 1.

[115] A. W. Unterberg, J. Stover, B. Kress, K. L. Kiening, Neuroscience 2004, 129, 1021.

[116] M. T. Fitch, J. Silver, Exp. Neurol. 1997, 148, 587.

[117] S. F. Bernatchez, P. J. Parks, D. F. Gibbons, Biomaterials 1996, 17, 2077.

[118] J. E. Sanders, C. E. Stiles, C. L. Hayes, J. Biomed. Mater. Res. 2000, 52, 231.

[119] C. S. Chen, J. Tan, J. Tien, Annu. Rev. Biomed. Eng. 2004, 6, 275.

[120] C. S. Chen, M. Mrksich, S. Huang, G. M. Whitesides, D. E. Ingber, Science 1997, 276, 1425.

[121] Y.-T. Kim, V. K. Haftel, S. Kumar, R. V. Bellamkonda, Biomaterials 2008, 29, 3117.

[122] L. Bekar, W. Libionka, G.-F. Tian, Q. Xu, A. Torres, X. Wang, D. Lovatt, E. Williams, T. Takano, J. Schnermann, R. Bakos, M. Nedergaard, Nat. Med. 2008, 14, 75 .

[123] M. L. Kringelbach, N. Jenkinson, S. L. F. Owen, T. Z. Aziz, Nat. Rev. Neurosci. 2007, 8, 623.

[124] J. T. Moyer, S. F. Danish, J. Neurosci. 2007, 27, 1799.

[125] J. S. Perlmutter, J. W. Mink, Annu. Rev. Neurosci. 2006, 29, 229.

[126] J. L. Vitek, Mov. Disord. 2002, 17, S69.

[127] J. O. Dostrovsky, R. Levry, J. P. Wu, W. D. Hutchison, R. R. Tasker, A. M. Lozano, J. Neurophysiol. 2000, 84, 570.

[128] C. Beurrier, B. Bioulac, J. Audin, C. Hammond, J. Neurophysiol. 2001, 85 , 1351.

[129] C. B. McCracken, A. A. Grace, J. Neurosci. 2007, 27, 12601.

[130] G. Peleg, A. Lewis, M. Linial, L. M. Loew, Proc. Natl. Acad. Sci. USA 1999, 96, 6700 .

[131] J. A. Kloepfer, N. Cohen, J. L. Nadeau, J. Phys. Chem. B 2004, 108, 17042.

[132] V. Salnikov, Y. O. Lukyanenko, C. A. Frederick, W. J. Lederer, V. Lukyanenko, Biophys. J. 2007, 92, 1058.

[133] J. Winter, C. Schmidt, B. Korgel, Optimization of quantum dot-Nerve cell interfaces, presented at Symposium on Quantum Dots, Nanoparticles and Nanowires held at the 2003 MRS Fall Meeting, Boston, MA, December 01-05, 2003.

[134] V. Nechyporuk-Zloy, C. Stock, H. Schillers, H. Oberleithner, A. Schwab, Am. J. Physiol. Cell Physiol. 2006, 291, C266.

[135] S. Ramachandran, N. E. Merrill, R. H. Blick, D. W. V. D. Weide, Biosens. Bioelectron. 2005, 20, 2173.
[136] J. L. Nadeau, S. J. Clarke, C. A. Hollmann, D. M. Bahcheli, IEEE, Quantum dot-FRET systems for imaging of neuronal action potentials, presented at 28th Annual International Conference of the IEEE-Engineeringin-Medicine-and-Biology-Society, New York, NY, August 30-September 03, 2006

[137] G. Oberdorster, E. Oberdorster, J. Oberdorster, Environ. Health Perspect. 2005, 113, 823.

[138] E. Oberdorster, Environ. Health Perspect. 2004, 112, 1058.

[139] C. Kirchner, T. Liedl, S. Kudera, T. Pellegrino, A. Munoz Javier, H. E. Gaub, S. Stolzle, N. Fertig, W. J. Parak, Nano Lett. 2005, 5, 331.

[140] S. Clarke, J. Nadeau, D. Bahcheli, Z. Zhang, C. Hollmann, Conf. Proc. IEEE Eng. Med. Biol. Soc. 2005, 1, 504

[141] J. Lovric, S. J. Cho, F. M. Winnik, D. Maysinger, Chem. Biol. 2005, 12, 1227.

[142] A. M. Derfus, W. C. W. Chan, S. N. Bhatia, Nano Lett. 2004, 4, 11.

[143] A. A. Shvedova, V. Castranova, E. R. Kisin, D. Schwegler-Berry, A. R. Murray, V. Z. Gandelsman, A. Maynard, P. Baron, J. Toxicol. Environ Health A 2003, 66, 1909.

[144] C. W. Lam, J. T. James, R. McCluskey, R. L. Hunter, Toxicol. Sci. 2004, 77 126

[145] D. B. Warheit, B. R. Laurence, K. L. Reed, D. H. Roach, G. A. Reynolds, T. R. Webb, Toxicol. Sci. 2004, 77, 117.

[146] N. W. Shi Kam, T. C. Jessop, P. A. Wender, H. Dai, J. Am. Chem. Soc. 2004 126, 6850.

[147] Y. Sato, A. Yokoyama, K. Shibata, Y. Akimoto, S. Ogino, Y. Nodasaka, T. Kohgo, K. Tamura, T. Akasaka, M. Uo, K. Motomiya, B. Jeyadevan, M. Ishiguro, R. Hatakeyama, F. Watari, K. Tohji, Mol. Biosyst. 2005, 1, 176.

[148] D. Cui, F. Tian, C. S. Ozkan, M. Wang, H. Gao, Toxicol. Lett. 2005, 155, 73.

[149] S. K. Manna, S. Sarkar, J. Barr, K. Wise, E. V. Barrera, O. Jejelowo, A. C. Rice-Ficht, G. T. Ramesh, Nano Lett. 2005, 5, 1676.

[150] C. M. Sayes, F. Liang, J. L. Hudson, J. Mendez, W. Guo, J. M. Beach, V. C. Moore, C. D. Doyle, J. L. West, W. E. Billups, K. D. Ausman, V. L. Colvin, Toxicol. Lett. 2006, 161, 135.

[15 1] M. P. Mattson, R. C. Haddon, A. M. Rao, J. Mol. Neurosci. 2000, 14, 175

[152] V. Lovat, D. Pantarotto, L. Lagostena, B. Cacciari, M. Grandolfo, M. Righi, G. Spalluto, M. Prato, L. Ballerini, Nano Lett. 2005, 5, 1107.

[153] M. A. Correa-Duarte, N. Wagner, J. Rojas-Chapana, C. Morsczeck, M. Thie, M. Giersig, Nano Lett. 2004, 4, 2233.

[154] J. A. Rojas-Chapana, M. Giersig, J. Nanosci. Nanotechnol. 2006, 6, 316.

[155] B. Diaz, C. Sanchez-Espinel, M. Arruebo, J. Faro, E. de Miguel, S. Magadan, C. Yague, R. Fernandez-Pacheco, M. R. Ibarra, J. Santamaria, A. Gonzalez-Fernandez, Small 2008, 4, 2025

[156] N. G. Bastus, E. Casals, S. Vazquez-Campos, V. Puntes, Nanotoxicology 2008, 2, 99

[157] M. J. D. Clift, B. Rothen-Rutishauser, D. M. Brown, R. Duffin, K. Donaldson, L. Proudfoot, K. Guy, V. Stone, Toxicol. Appl. Pharmacol. 2008, 232, 418.

[158] C. M. Sayes, A. M. Gobin, K. D. Ausman, J. Mendez, J. L. West, V. L. Colvin, Biomaterials 2005, 26, 7587.

[159] L. H. Ding, J. Stilwell, T. T. Zhang, O. Elboudwarej, H. J. Jiang, J. P. Selegue, P. A. Cooke, J. W. Gray, F. Q. F. Chen, Nano Lett. 2005, 5, 2448.

[160] J. R. Gurr, A. S. S. Wang, C. H. Chen, K. Y. Jan, Toxicology 2005, 213, 66

[161] W. S. Lin, Y. W. Huang, X. D. Zhou, Y. F. Ma, Toxicol. Appl. Pharmacol. 2006, 217, 252

[162] C. Buzea, I. I. Pacheco, K. Robbie, Biointerphases 2007, 2, MR17.

[163] H. C. Fischer, W. C. W. Chan, Curr. Opin. Biotechnol. 2007, 18, 565.

[164] M. A. Dobrovolskaia, S. E. McNeil, Nat. Nano 2007, 2, 469

[165] R. Duncan, L. Izzo, Adv. Drug Deliv. Rev. 2005, 57, 2215.

[166] S. Dokka, D. Toledo, X. G. Shi, V. Castranova, Y. Rojanasakul, Pharm. Res. 2000, 17, 521

[167] H. L. Wong, A. M. Rauth, R. Bendayan, J. L. Manias, M. Ramaswamy, Z. S Liu, S. Z. Erhan, X. Y. Wu, Pharm. Res. 2006, 23, 1574.

[168] R. FernandezUrrusuno, E. Fattal, J. M. Rodrigues, J. Feger, P. Bedossa, P. Couvreur, J. Biomed. Mater. Res. 1996, 31, 401. 
[169] B. Heurtault, P. Saulnier, B. Pech, J. E. Proust, J. P. Benoit, Biomaterials 2003, 24, 4283.

[170] J. Lovric, H. S. Bazzi, Y. Cuie, G. R. Fortin, F. M. Winnik, D. Maysinger, J. Mol. Med. 2005, 83, 377.

[171] W. H. Chan, N. H. Shiao, P. Z. Lu, Toxicol. Lett. 2006, 167, 191.

[172] A. O. Choi, S. J. Cho, J. Desbarats, J. Lovric, D. Maysinger, J. Nanobiotechnol. 2007, 5, 1.

[173] D. Maysinger, M. Behrendt, M. Lalancette-Hebert, J. Kriz, Nano Lett. 2007, 7, 2513.

[174] T. Q. Vu, R. Maddipati, T. A. Blute, B. J. Nehilla, L. Nusblat, T. A. Desai, Nano Lett. 2005, 5, 603.

[175] M. P. Waalkes, Mutat. Res. 2003, 533, 107.

[176] A. Hartwig, T. Schwerdtle, Toxicol. Lett. 2002, 127, 47.

[177] G. S. Shukla, R. S. Srivastava, S. V. Chandra, Fundam. Appl. Toxicol. 1988, 11, 229.

[178] A. O. Choi, S. E. Brown, M. Szyf, D. Maysinger, J. Mol. Med. 2007, 86, 291.

[179] T. Zhang, J. L. Stilwell, D. Gerion, L. Ding, O. Elboudwarej, P. A. Cooke, J. W. Gray, A. P. Alivisatos, F. F. Chen, Nano Lett. 2006, 6, 800.

[180] E. Jan, S. J. Byrne, M. Cuddihy, A. M. Davies, Y. Volkov, Y. K. Gun'ko, N. A. Kotov, ACS Nano 2008, 2, 928.

[181] S. J. Cho, D. Maysinger, M. Jain, B. Roder, S. Hackbarth, F. M. Winnik, Langmuir 2007, 23, 1974.

[182] A. C. Samia, X. Chen, C. Burda, J. Am. Chem. Soc. 2003, 125, 15736.

[183] V. A. Sinani, D. S. Koktysh, B.-G. Yun, R. L. Matts, T. C. Pappas, M. Motamedi, S. T. Thomas, N. A. Kotov, Nano Lett. 2003, 3, 1177.

[184] A. Hoshino, K. Fujioka, T. Oku, M. Suga, Y. F. Sasaki, T. Ohta, M. Yasuhara, K. Suzuki, K. Yamamoto, Nano Lett. 2004, 4, 2163.

[185] A. Hoshino, N. Manabe, K. Fujioka, K. Suzuki, M. Yasuhara, K. Yamamoto, J. Artif. Organs 2007, 10, 149.

[186] A. Shiohara, A. Hoshino, K. Hanaki, K. Suzuki, K. Yamamoto, Microbiol. Immunol. 2004, 48, 669.

[187] M. L. Schipper, N. Nakayama-Ratchford, C. R. Davis, N. W. S. Kam, P. Chu, Z. Liu, X. Sun, H. Dai, S. S. Gambhir, Nat. Nanotechnol. 2008, 3, 216.

[188] W. Yang, P. Thordarson, J. J. Gooding, S. P. Ringer, F. Braet, Nanotechnology 2007, 18, 412001/1.

[189] G. Angelini, S. Boncompagni, P. De Maria, M. De Nardi, A. Fontana, C. Gasbarri, E. Menna, Carbon 2007, 45, 2479.

[190] M. Prato, K. Kostarelos, A. Bianco, Acc. Chem. Res. 2008, 41, 60.

[191] J. Guo, X. Zhang, Q. Li, W. Li, Nucl. Med. Biol. 2007, 34, 579.

[192] Y. Sato, A. Yokoyama, K.-I. Shibata, F. Watari, K. Tohji, Tanso 2006, 225, 364.

[193] J. M. Ashcroft, K. B. Hartman, Y. Mackeyev, C. Hofmann, S. Pheasant, L. B. Alemany, L. J. Wilson, Nanotechnology 2006, 17, 5033.

[194] J. C. Carrero-Sanchez, A. L. Elias, R. Mancilla, G. Arrellin, H. Terrones, J. P. Laclette, M. Terrones, Nano Lett. 2006, 6, 1609.

[195] N. W. S. Kam, Z. Liu, H. Dai, Angew. Chem. Int. Ed. 2006, 45, 577

[196] N. W. S. Kam, Z. Liu, H. Dai, J. Am. Chem. Soc. 2005, 127, 12492

[197] H. Dumortier, S. Lacotte, G. Pastorin, R. Marega, W. Wu, D. Bonifazi, J.-P. Briand, M. Prato, S. Muller, A. Bianco, Nano Lett. 2006, 6, 3003.

[198] V. E. Kagan, Y. Y. Tyurina, V. A. Tyurin, N. V. Konduru, A. I. Potapovich, A. N. Osipov, E. R. Kisin, D. Schwegler-Berry, R. Mercer, V. Castranova, A. A. Shvedova, Toxicol. Lett. 2006, 165, 88.

[199] A. Magrez, S. Kasas, V. Salicio, N. Pasquier, J. W. Seo, M. Celio, S. Catsicas, B. Schwaller, L. Forro, Nano Lett. 2006, 6, 1121.

[200] H. Hu, Y. Ni, V. Montana, R. C. Haddon, V. Parpura, Nano Lett. 2004, 4, 507.

[201] Y. Ni, H. Hu, E. B. Malarkey, B. Zhao, V. Montana, R. C. Haddon, V. Parpura, J. Nanosci. Nanotechnol. 2005, 5, 1707.

[202] H. Hu, Y. Ni, S. K. Mandal, V. Montana, B. Zhao, R. C. Haddon, V. Parpura, J. Phys. Chem. B 2005, 109, 4285.

[203] T. C. Pappas, W. M. S. Wickramanyake, E. Jan, M. Motamedi, M. Brodwick, N. A. Kotov, Nano Lett. 2007, 7, 513.

[204] K. Wang, H. A. Fishman, H. Dai, J. S. Harris, Nano Lett. 2006, 6, 2043.

[205] C. J. Smith, B. J. Shaw, R. D. Handy, Aquat. Toxicol. 2007, 82, 94.
[206] A. V. Liopo, M. P. Stewart, J. Hudson, J. M. Tour, T. C. Pappas, J. Nanosci. Nanotechnol. 2006, 6, 1365

[207] A. Helland, P. Wick, A. Koehler, K. Schmid, C. Som, Environ. Health Perspect. 2007, 115, 1125.

[208] A. Mazzatenta, M. Giugliano, S. Campidelli, L. Gambazzi, L. Businaro, H. Markram, M. Prato, L. Ballerini, J. Neurosci. 2007, 27, 6931.

[209] A. Huczko, H. Lange, Fullerene Sci. Technol. 2001, 9, 247.

[210] C. S. Sharma, S. Sarkar, A. Periyakaruppan, J. Barr, K. Wise, R. Thomas, B. L. Wilson, G. T. Ramesh, J. Nanosci. Nanotechnol. 2007, 7, 2466.

[211] K. Pulskamp, S. Diabate, H. F. Krug, Toxicol. Lett. 2007, 168, 58.

[212] M. Bottini, S. Bruckner, K. Nika, N. Bottini, S. Bellucci, A. Magrini, A. Bergamaschi, T. Mustelin, Toxicol. Lett. 2006, 160, 121.

[213] J. M. Worle-Knirsch, K. Pulskamp, H. F. Krug, Nano Lett. 2006, 6, 1261.

[214] N. A. Monteiro-Riviere, R. J. Nemanich, A. O. Inman, Y. Y. Wang, J. E. Riviere, Toxicol. Lett. 2005, 155, 377.

[215] D. Pantarotto, J. P. Briand, M. Prato, A. Bianco, Chem. Commun. (Camb.) 2004, 16.

[216] D. Pantarotto, R. Singh, D. McCarthy, M. Erhardt, J. P. Briand, M. Prato, K. Kostarelos, A. Bianco, Angew. Chem. Int. Ed. Engl. 2004, 43, 5242.

[217] A. Nimmagadda, K. Thurston, M. U. Nollert, P. S. McFetridge, J. Biomed. Mater. Res. 2006, 76, 614 .

[218] F. Tian, D. Cui, H. Schwarz, G. G. Estrada, H. Kobayashi, Toxicol. In Vitro 2006, 20, 1202.

[219] A. M. Rajnicek, S. Britland, C. D. McCaig, J. Cell Sci. 1997, 110, 2905

[220] G. P. Dillon, X. Yu, R. V. Bellamkonda, J. Biomed. Mater. Res. 2000, 51, 510.

[221] G. Jia, H. Wang, L. Yan, X. Wang, R. Pei, T. Yan, Y. Zhao, X. Guo, Environ. Sci. Technol. 2005, 39, 1378

[222] P. Wick, P. Manser, L. K. Limbach, U. Dettlaff-Weglikowska, F. Krumeich, S. Roth, W. J. Stark, A. Bruinink, Toxicol. Lett. 2007, 168, 121.

[223] P. M. Raja, J. Connolley, G. P. Ganesan, L. Ci, P. M. Ajayan, O. Nalamasu, D. M. Thompson, Toxicol. Lett. 2007, 169, 51

[224] D. H. Kim, D. C. Martin, Biomaterials 2006, 27, 3031.

[225] D. A. Eavarone, X. J. Yu, R. V. Bellamkonda, J. Biomed. Mater. Res. 2000, 51,10

[226] J. M. Saul, A. Annapragada, J. V. Natarajan, R. V. Bellamkonda, J. Control. Release 2003, 92, 49

[227] J. M. Saul, A. V. Annapragada, R. V. Bellamkonda, J. Control. Release 2006, 114, 277

[228] J. Kreuter, Adv. Drug Deliv. Rev. 2001, 47, 65.

[229] Y. Xie, L. Y. Ye, X. B. Zhang, W. Cui, J. N. Lou, T. Nagai, X. P. Hou, J. Control. Release 2005, 105, 106.

[230] D. Papahadjopoulos, T. M. Allen, A. Gabizon, E. Mayhew, K. Matthay, S. K. Huang, K. D. Lee, M. C. Woodle, D. D. Lasic, C. Redemann, F. J. Martin, Proc. Natl. Acad. Sci. USA 1991, 88, 11460.

[231] A. Weissenbock, M. Wirth, F. Gabor, J. Control. Release 2004, 99, 383.

[232] D. J. Bharali, I. Klejbor, E. K. Stachowiak, P. Dutta, I. Roy, N. Kaur, E. J. Bergey, P. N. Prasad, M. K. Stachowiak, Proc. Natl. Acad Sci. USA 2005, 102, 11539.

[233] L. L. Zou, L. Huang, R. L. Hayes, C. Black, Y. H. Qiu, J. R. Perez-Polo, W. Le, G. L. Clifton, K. Yang, Gene Ther. 1999, 6, 994.

[234] S. Jin, K. M. Ye, Biotechnol. Prog. 2007, 23, 32.

[235] A. Bianco, K. Kostarelos, M. Prato, Curr. Opin. Chem. Biol. 2005, 9, 674.

[236] C. E. Thomas, A. Ehrhardt, M. A. Kay, Nat. Rev. Genet. 2003, 4, 346

[237] K. Yang, G. L. Clifton, R. L. Hayes, J. Neurotrauma 1997, 14, 281.

[238] J. Panyam, V. Labhasetwar, Adv. Drug Deliv. Rev. 2003, 55, 329.

[239] J. Kreuter, D. Shamenkov, V. Petrov, P. Ramge, K. Cychutek, C. KochBrandt, R. Alyautdin, J. Drug Target 2002, 10, 317.

[240] W. Sun, C. Xie, H. Wang, Y. Hu, Biomaterials 2004, 25, 3065.

[241] W. Sun, H. Wang, C. Xie, Y. Hu, X. Yang, H. Xu, J. Control. Release 2006, 115, 259.

[242] G. Liu, P. Men, P. L. Harris, R. K. Rolston, G. Perry, M. A. Smith, Neurosci. Lett. 2006, 406, 189

[243] W. Lu, Y. Zhang, Y. Tan, K. Hu, X. Jiang, S. Fu, J. Control. Release 2005, 107, 428 
[244] W. Lu, Q. Sun, J. Wan, Z. She, X.-G. Jiang, Cancer Res. 2006, 66, 11878.

[245] N. Tagmatarchis, H. Shinohara, Mini Rev. Med. Chem. 2001, 1, 339.

[246] E. Tóth, R. D. Bolskar, A. Borel, G. González, L. Helm, A. E. Merbach, B. Sitharaman, L. J. Wilson, J. Am. Chem. Soc. 2005, 127, 799.

[247] P. P. Fatouros, F. D. Corwin, Z.-J. Chen, W. C. Broaddus, J. L. Tatum, B. Kettenmann, Z. Ge, H. W. Gibson, J. L. Russ, A. P. Leonard, J. C. Duchamp, H. C. Dorn, Radiology 2006, 240, 756.

[248] E. H. Lo, T. Dalkara, M. A. Moskowitz, Nat. Rev. Neurosci. 2003, 4, 399.

[249] J. X. Wilson, A. W. Gelb, J. Neurosurg. Anesthesiol. 2002, 14, 66.

[250] R. Raghupathi, D. I. Graham, T. K. McIntosh, J. Neurotrauma 2000, 17, 927.

[251] A. Verma, J. Head. Trauma Rehabil. 2000, 15, 1149.

[252] L. L. Dugan, D. M. Turetsky, C. Du, D. Lobner, M. Wheeler, C. R. Almli, C. K.-F. Shen, T.-Y. Luh, D. W. Choi, T.-S. Lin, Proc. Natl. Acad. Sci. USA 1997, 94, 9434.

[253] H. Jin, W. Q. Chen, X. W. Tang, L. Y. Chiang, C. Y. Yang, J. V. Schloss, J. Y. Wu, J. Neurosci. Res. 2000, 62,600.

[254] L. L. Dugan, E. G. Lovett, K. L. Quick, J. Lotharius, T. T. Lin, K. L. O’Malley, Parkinsonism Relat. Disord. 2001, 7, 243.

[255] J. D. Hartgerink, E. Beniash, S. I. Stupp, Science 2001, 294, 1684.

[256] K. L. Niece, J. D. Hartgerink, J. J. Donners, S. I. Stupp, J. Am. Chem. Soc. 2003, 125, 7146.

[257] G. A. Silva, C. Czeisler, K. L. Niece, E. Beniash, D. A. Harrington, J. A. Kessler, S. I. Stupp, Science 2004, 303, 1352.

[258] T. D. B. Nguyen-Vu, H. Chen, A. M. Cassell, R. Andrews, M. Meyyappan, J. Li, Small 2006, 2, 89.

[259] R. G. Ellis-Behnke, Y. X. Liang, S. W. You, D. K. Tay, S. Zhang, K. F. So, G. E. Schneider, Proc. Natl. Acad. Sci. 2006, 103, 5054.

[260] T. C. Holmes, Trends Biotechnol. 2002, 20, 16.

[261] S. I. Stupp, V. V. LeBonheur, K. Walker, L. S. Li, K. E. Huggins, M. Keser, A. Amstutz, Science 1997, 276, 384.

[262] S. Zhang, Nat. Biotechnol. 2003, 21, 1171.

[263] S. Zhang, D. M. Marini, W. Hwang, S. Santoso, Curr. Opin. Chem. Biol. 2002, 6, 865 .

[264] J. D. Hartgerink, E. Beniash, S. I. Stupp, Proc. Natl. Acad. Sci. USA 2002, 99, 5133.

[265] A. Hoshino, K. Fujioka, T. Oku, S. Nakamura, M. Suga, Y. Yamaguchi, K. Suzuki, M. Yasuhara, K. Yamamoto, Microbiol. Immunol. 2004, 48, 985.

[266] J. Kim, J. E. Lee, S. H. Lee, J. H. Yu, J. H. Lee, T. G. Park, T. Hyeon, Adv. Mater. 2008, 20, 478.

[267] Y.-W. Jun, J.-W. Seo, J. Cheon, Acc. Chem. Res. 2008, 41, 179.

[268] H. B. Na, J. H. Lee, K. An, Y. I. Park, M. Park, I. S. Lee, D.-H. Nam, S. T. Kim, S.-H. Kim, S.-W. Kim, K.-H. Lim, K.-S. Kim, S.-O. Kim, T. Hyeon, Angew. Chem. Int. Ed. 2007, 46, 5397.

[269] H. L. Liu, J. H. Wu, J. H. Min, Y. K. Kim, J. Appl. Phys. 2008, 103, 07D529/1.

[270] P. R. Lockman, R. J. Mumper, M. A. Khan, D. D. Allen, Drug Dev. Ind. Pharm. 2002, 28, 1.

[271] K. Karunya, M. D. D. Kandimalla, J. Pharm. Sci. 2005, 94, 613.

[272] F. Cengelli, D. Maysinger, F. Tschudi-Monnet, X. Montet, C. Corot, A. Petri-Fink, H. Hofmann, L. Juillerat-Jeanneret, J. Pharmacol. Exp. Ther. 2006, 318, 108.

[273] C. Tu, Y. Yang, M. Gao, Nanotechnology 2008, 19, 105601/1.

[274] F. Hu, Z. Li, C. Tu, M. Gao, J. Colloid Interface Sci. 2007, 311, 469.

[275] A. Jordan, K. Maier-Hauff, J. Nanosci. Nanotechnol. 2007, 7, 4604.

[276] C. H. Liu, Z. You, J. Ren, Y. R. Kim, K. Eikermann-Haerter, P. K. Liu, FASEB J. 2008, 22, 1193.

[277] B. Chertok, A. E. David, Y. Huang, V. C. Yang, J. Control. Release 2007, 122, 315.

[278] G. R. Reddy, M. S. Bhojani, P. McConville, J. Moody, B. A. Moffat, D. E. Hall, G. Kim, Y.-E. L. Koo, M. J. Woolliscroft, J. V. Sugai, T. D. Johnson, M. A. Philbert, R. Kopelman, A. Rehemtulla, B. D. Ross, Clin. Cancer Res. 2006, 12, 6677.
[279] A. Jordan, R. Scholz, K. Maier-Hauff, F. K. H. van Landeghem, N. Waldoefner, U. Teichgraeber, J. Pinkernelle, H. Bruhn, F. Neumann, B. Thiesen, A. von Deimling, R. Felix, J. Neurooncol. 2006, 78, 7.

[280] B. Chertok, B. A. Moffat, A. E. David, F. Yu, C. Bergemann, B. D. Ross, V. C. Yang, Biomaterials 2007, 29, 487.

[281] T. K. Jain, M. K. Reddy, M. A. Morales, D. L. Leslie-Pelecky, V. Labhasetwar, Mol. Pharm. 2008, 5, 316 .

[282] J. S. Kim, T.-J. Yoon, K. N. Yu, B. G. Kim, S. J. Park, H. W. Kim, K. H. Lee, S. B. Park, J.-K. Lee, M. H. Cho, Toxicol. Sci. 2006, 89, 338.

[283] A. Watson, X. Wu, M. Bruchez, Biotechniques 2003, 34, 296.

[284] F. Tokumasu, J. Dvorak, J. Microsc. 2003, 211, 256.

[285] J. K. Jaiswal, E. R. Goldman, H. Mattoussi, S. M. Simon, Nat. Methods 2004, 1, 73.

[286] M. E. Akerman, W. C. Chan, P. Laakkonen, S. N. Bhatia, E. Ruoslahti, Proc. Natl. Acad. Sci. USA 2002, 99, 12617.

[287] W. C. Chan, D. J. Maxwell, X. Gao, R. E. Bailey, M. Han, S. Nie, Curr. Opin. Biotechnol. 2002, 13, 40.

[288] X. Gao, S. Nie, Trends Biotechnol. 2003, 21, 371.

[289] S. Pathak, E. Cao, M. C. Davidson, S. Jin, G. A. Silva, J. Neurosci. 2006, 26, 1893.

[290] M. Dahan, S. Levi, C. Luccardini, P. Rostaing, B. Riveau, A. Triller, Science 2003, 302, 442.

[291] B. Zuber, I. Nikonenko, P. Klauser, D. Muller, J. Dubochet, Proc Natl. Acad. Sci. 2005, 102, 19192.

[292] M. J. Dalby, N. Gadegaard, M. O. Riehle, C. D. W. Wilkinson, A. S. G Curtis, Int. J. Biochem. Cell Biol. 2004, 36, 2005.

[293] V. Nurcombe, M. Aumailley, R. Timpl, D. Edgar, Eur.J. Biochem. 1989, 180 9.

[294] G. A. Abrams, S. L. Goodman, P. F. Nealey, M. Franco, C. J. Murphy, Cell Tissue Res. 2000, 299, 39.

[295] A. M. Taylor, S. W. Rhee, C. H. Tu, D. H. Cribbs, C. W. Cotman, N. L. Jeon, Langmuir 2003, 19, 1551.

[296] M. J. Mahoney, R. R. Chen, J. Tan, W. M. Saltzman, Biomaterials 2004, 26, 771.

[297] P. G. Gross, L. P. Weiner, E. P. Kartalov, A. Scherer, Crit. Rev. Neurobiol. 2005, 17, 119

[298] S. W. Rhee, A. M. Taylor, C. H. Tu, D. H. Cribbs, C. W. Cotman, N. L. Jeon, Lab Chip 2005, 5, 102.

[299] L. J. Millet, M. E. Stewart, J. V. Sweedler, R. G. Nuzzo, M. U. Gillette, Lab Chip 2007, 7, 987.

[300] S. Lang, A. C. von Philipsborn, A. Bernard, F. Bonhoeffer, M. Bastmeyer Anal. Bioanal. Chem. 2008, 390, 809.

[301] B. Vahidi, J. W. Park, H. J. Kim, N. L. Jeon, J. Neurosci. Methods 2008, 170 188.

[302] C. J. Wang, X. Li, B. Lin, S. Shim, G.-I. Levchenko, A. Ming, Lab Chip 2008 8, 227.

[303] J. W. Park, B. Vahidi, A. M. Taylor, S. W. Rhee, N. L. Jeon, Nat. Protoc. 2006 1, 2128.

[304] D. R. Reyes, E. M. Perruccio, S. P. Becerra, L. E. Locascio, M. Gaitan Langmuir 2004, 20, 8805.

[305] S. P. Forry, D. R. Reyes, M. Gaitan, L. E. Locascio, Langmuir 2006, 22, 5770

[306] N. Z. Mehenti, H. A. Fishman, S. F. Bent, Biomed. Microdevices 2007, 9, 579.

[307] L. Rowe, M. Almasri, K. Lee, N. Fogleman, G. J. Brewer, Y. Nam, B. C. Wheeler, J. Vukasinovic, A. Glezer, A. B. Frazier, Lab Chip 2007, 7, 475.

[308] C. J. Emmett, W. Jaquesberg, P. J. Seeley, Neuroscience 1990, 38, 213.

[309] S. J. A. Davies, P. M. Field, G. Raisman, Exp. Neurol. 1996, 142, 203.

[310] A. Beric, P. J. Kelly, A. Rezai, D. Sterio, A. Mogilner, M. Zonenshayn, B. Kopell, Stereotact. Funct. Neurosurg. 2001, 77, 73.

[311] D. W. M. Arrigan, Analyst 2004, 129, 1157.

[312] J. M. Corey, B. C. Wheeler, G. J. Brewer, IEEE Trans. Biomed. Eng. 1996, 43, 944.

[313] Y.-Y. Luk, M. Kato, M. Mrksich, Langmuir 2000, 16, 9604.

[314] C. R. Jenney, J. M. Anderson, J. Biomed. Mater. Res. 1999, 44, 206. 
[315] E. Ostuni, R. G. Chapman, R. E. Holmlin, S. Takayama, G. M. Whitesides, Langmuir 2001, 17, 5605.

[316] R. Singhvi, A. Kumar, G. P. Lopez, G. N. Stephanopoulos, D. I. C. Wang, G. M. Whitesides, D. E. Ingber, Science 1994, 264, 696.

[317] E. Ostuni, B. A. Grzybowski, M. Mrksich, C. S. Roberts, G. M. Whitesides, Langmuir 2003, 19, 1861.

[318] J. B. Brzoska, I. B. Azouz, F. Rondelez, Langmuir 1994, 10, 4367.

[319] Q.-Y. Liu, M. Coulombe, J. Dumm, K. M. Shaffer, A. E. Schaffner, J. L. Barker, J. J. Pancrazio, D. A. Stenger, W. Ma, Dev. Brain Res. 2000, 120, 223.

[320] C. Wyart, C. Ybert, L. Bourdieu, C. Herr, C. Prinz, D. Chatenay, J. Neurosci. Methods 2002, 117, 123.

[321] L. Kam, W. Shain, J. N. Turner, R. Bizios, Biomaterials 2001, 22, 1049.

[322] K. K. Parker, A. L. Brock, C. Brangwynne, R. J. Mannix, N. Wang, E. Ostuni, N. A. Geisse, J. C. Adams, G. M. Whitesides, D. E. Ingber, FASEB 2002, 16, 1195.

[323] S. Rohr, R. Flückiger-Labrada, J. P. Kucera, Eur. J. Physiol. 2003, 446, 125.

[324] H. G. Craighead, S. W. Turner, R. C. Davies, C. James, A. M. Perez, P. M. St, John, M. Isaacson, W. Shain, J. N. Turner, G. J. Banker, Biomed. Microdev. 1998, 1, 49.

[325] B. Wojciak-Stothard, M. Denyer, M. Mishra, R. A. Brown, In Vitro Cell. Dev. Biol. 1997, 33, 110.

[326] A. Curtis, C. Wilkinson, Biomaterials 1997, 18, 1573.

[327] F. Johansson, P. Carlbergb, N. Danielsenc, L. Monteliusb, M. Kanjea, Biomaterials 2006, 27, 1251.

[328] D. M. Brunette, J. Dent. Res. 1995, 74, 406.

[329] P. Clark, P. Connolly, A. S. Curtis, J. A. Dow, C. D. Wilkinson, J. Cell Sci. 1991, 99, 73

[330] P. Clark, P. Connolly, A. S. G. Curtis, J. A. T. Dow, C. D. W. Wilkinson, Development 1990, 108, 635 .

[331] A. S. G. Curtis, M. Varde, J. Natl. Cancer Inst. 1964, 33, 15.

[332] E. T. Denbraber, J. E. Deruijter, L. A. Ginsel, A. F. von Recum, J. A. Jansen, Biomaterials 1996, 17, 2037.

[333] K. Matsuzaka, F. Walboomers, A. de Ruijter, J. A. Jansen, Clin. Oral Implants Res. 2000, 11, 325.

[334] A. F. von Recum, T. G. van Kooten, J. Biomater. Sci. Polym. Ed. 1995, 7, 181.

[335] P. Weiss, J. Exp. Zool. 1945, 100, 353.

[336] R. Sorkin, T. Gabay, P. Blinder, D. Baranes, E. Ben-Jacob, Y. Hanein, J. Neural Eng. 2006, 3, 95.

[337] S. Britland, H. Morgan, B. WojiakStodart, M. Riehle, A. Curtis, C. Wilkinson, Exp. Cell Res. 1996, 228, 313.

[338] C. Miller, S. Jeftinija, S. Mallapragada, Tissue Eng. 2001, 7, 705

[339] R. G. Flemming, C. J. Murphy, G. A. Abrams, S. L. Goodman, P. F. Nealey, Biomaterials 1999, 20, 573

[340] J. D. Foley, E. W. Grunwald, P. F. Nealey, C. J. Murphy, Biomaterials 2005, 26, 3639.

[341] L. S. Chou, J. D. Firth, V. J. Uitto, D. M. Brunette, J. Cell Sci. 1995, 108, 1563

[342] Y. W. Fan, F. Z. Cui, L. N. Chen, Y. Zhai, Q. Y. Xu, I. S. Lee, Appl. Surf. Sci. 2002, 187, 313.

[343] Y. W. Fan, F. Z. Cui, S. P. Hou, Q. Y. Xu, L. N. Chen, I. S. Lee, J. Neurosci. Methods 2002, 120, 17.

[344] S. Turner, L. Kam, M. Isaacson, H. G. Craighead, W. Shain, J. Turner, J. Vac. Sci. Technol. B 1997, 15, 2848.

[345] A. Teixeira, G. A. Abrams, P. J. Bertics, C. J. Murphy, P. F. Nealey, J. Cell Sci. 2003, 116, 1881

[346] A. S. G. Curtis, Eur. Cells Mater. 2004, 8, 27.

[347] B. T. D. Nguyen-Vu, H. Chen, A. M. Cassell, R. J. Andrews, M. Meyyappan, J. Li, IEEE Trans. Biomed. Eng. 2007, 54, 1121.

[348] B. S. Shim, P. Podsiadlo, D. G. Lilly, A. Agarwal, J. Lee, Z. Tang, S. Ho, P. Ingle, D. Paterson, W. Lu, N. A. Kotov, Nano Lett. 2007, 7, 3266.

[349] A. S. G. Curtis, N. Gadegaard, M. J. Dalby, M. O. Riehle, C. D. W. Wilkinson, G. Aitchison, IEEE Trans. Nanobiosci. 2004, 3, 61.
[350] M. J. Dalby, M. J. Biggs, N. Gadegaard, G. Kalna, C. D. W. Wilkinson, A. S. G. Curtis, J. Cell. Biochem. 2007, 100, 326.

[351] M. J. Dalby, D. Giannaras, M. O. Riehle, N. Gadegaard, S. Affrossman, A. S. G. Curtis, Biomaterials 2004, 25, 77.

[352] M. J. Dalby, M. O. Riehle, D. S. Sutherland, H. Agheli, A. S. G. Curtis, Eur. Cells Mater. 2005, 9, 1

[353] R. Biran, M. D. Noble, P. A. Tresco, Exp. Neurol. 2003, 184, 141.

[354] G. A. Brook, J. M. Lawrence, B. Shah, G. Raisman, Exp. Neurol. 1994, 126, 31.

[355] M. E. Manwaring, J. F. Walsh, P. A. Tresco, Biomaterials 2004, 25, 3631.

[356] J. F. Walsh, M. E. Manwaring, P. A. Tresco, Tissue Eng. 2005, 11, 1085.

[357] D. H. Kim, S. M. Richardson-Burns, J. L. Hendricks, C. Sequera, D. C. Martin, Adv. Funct. Mater. 2007, 17, 79.

[358] C. Vieu, F. Carcenac, A. Pepin, Y. Chen, M. Mejias, A. Lebib, L. Manin-Ferlazzo, L. Couraud, H. Launois, Appl. Surf. Sci. 2000, 164, 111.

[359] J. Norman, T. Desai, Ann. Biomed. Eng. 2006, 34, 89.

[360] K. B. Lee, S. J. Park, C. A. Mirkin, J. C. Smith, M. Mrksich, Science 2002, 295, 1702.

[361] S. F. Lyuksyutov, R. A. Vaia, P. B. Paramonov, S. Juhl, L. Waterhouse, R. M. Ralich, G. Sigalov, E. Sancaktar, Nat. Mater. 2003, 2, 468.

[362] Y. W. Fan, F. Z. Cui, S. P. Hou, Q. Y. Xu, L. N. Chen, I. S. Lee, J. Neurosci. Methods 2002, 120, 17.

[363] H. Ai, H. Meng, I. Ichinose, S. A. Jones, D. K. Mills, Y. M. Lvov, X. Qiao, J. Neurosci. Methods 2003, 128, 1.

[364] J. S. Mohammed, M. A. DeCoster, M. J. McShane, Biomacromolecules 2004, 5, 1745.

[365] F. Yang, R. Murugan, S. Wang, S. Ramakrishna, Biomaterials 2005, 26, 2603.

[366] Z. M. Huang, Y. Z. Zhang, M. Kotaki, S. Ramakrishna, Compos. Sci. Technol. 2003, 63, 2223.

[367] J. Y. Yang, D. H. Kim, J. L. Hendricks, M. Leach, R. Northey, D. C. Martin, Acta Biomater. 2005, 1, 125.

[368] J. Y. Yang, D. C. Martin, Sens. Actuators B-Chem. 2004, 101, 133.

[369] H. Shen, Y. T. Kim, R. Bellamkonda, S. Kumar, Abstr. Pap. Am. Chem. Soc. 2006, 231, 453-PMSE.

[370] Y. T. Kim, V. K. Haftel, S. Kumar, R. V. Bellamkonda, Biomaterials 2008, 29, 3117.

[371] Y. Katayama, R. Montenegro, T. Freier, R. Midha, J. S. Belkas, M. S. Shoichet, Biomaterials 2006, 27, 505

[372] D. W. Branch, B. C. Wheeler, G. J. Brewer, D. E. Leckband, IEEE Trans. Biomed. Eng. 2000, 47, 290.

[373] J. C. Chang, G. J. Brewer, B. C. Wheeler, Biosens. Bioelectron. 2001, 16, 527.

[374] H. Takano, J.-Y. Sul, M. L. Mazzanti, R. T. Doyle, P. G. Haydon, M. D. Porter, Anal. Chem. 2002, 74, 4640.

[375] B. C. Wheeler, J. M. Corey, G. J. Brewer, D. W. Branch, J. Biomech. Eng. 1999, 121, 73.

[376] M. Matsuzawa, K. Umemura, D. Beyer, K. Sugoka, W. Knoll, Thin Solid Films 1997, 305, 74

[377] a) A. Offenhausser, C. Sprossler, M. Matsuzawa, W. Knoll, Neurosci. Lett. 1997, 223, 9. b) Enabling Technologies for Cultured Neural Networks (Eds: D. A, Stenger, T. M. McKenna) Academic Press, San Diego 1994, pp. 284.

[378] M. Matsuzawa, S. Tokumitsu, W. Knoll, P. Liesi, J. Neurosci. Res. 1998, 53, 114.

[379] C. Wilkinson, A. Curtis, Phys. World 1999, 12, 45.

[380] G. Bayer, A. Borck, WO Patent Application 2004-EP5550, 2004112891, 2004.

[381] X. Zhang, S. Prasad, S. Niyogi, A. Morgan, M. Ozkan, C. S. Ozkan, Sens. Actuators B-Chem. 2005, 106, 843.

[382] W. Shain, L. Spataro, J. Dilgen, K. Haverstick, S. Retterer, M. Isaacson, M. Saltzman, J. N. Turner, IEEE Trans. Neural Syst. Rehabil. Eng. 2003, 11, 186.

[383] Y. Zhong, R. V. Bellamkonda, Brain Res. 2007, 1148, 15.

[384] G. Decher, Science 1997, 277, 1232.

[385] W. He, R. V. Bellamkonda, Biomaterials 2005, 26, 2983. 
[386] Z. Y. Tang, Y. Wang, P. Podsiadlo, N. A. Kotov, Adv. Mater. 2006, 18, 3203.

[387] E. Jan, N. A. Kotov, Nano Lett. 2007, 7, 1123.

[388] W. He, G. C. McConnell, R. V. Bellamkonda, J. Neural Eng. 2006, 3, 316.

[389] N. Benkirane-Jessel, P. Schwinte, P. Falvey, R. Darcy, Y. Haikel, P. Schaaf, J. C. Voegel, J. Ogier, Adv. Funct. Mater. 2004, 14, 174.

[390] N. Benkirane-Jessel, P. Lavalle, F. Meyer, F. Audouin, B. Frisch, P. Schaaf, J. Ogier, G. Decher, J. C. Voegel, Adv. Mater. 2004, 16, 1507.

[391] R. A. Green, N. H. Lovell, G. G. Wallace, L. A. Poole-Warren, Biomaterials 2008, 29, 3393.

[392] M. R. Abidian, D. H. Kim, D. C. Martin, Adv. Mater. 2006, 18, 405.

[393] S. M. Richardson-Burns, J. L. Hendricks, B. Foster, L. K. Povlich, D. H. Kim, D. C. Martin, Biomaterials 2007, 28, 1539.

[394] M. R. Abidian, D. C. Martin, Biomaterials 2008, 29, 1273.

[395] J. S. Foos, S. M. Erker, Mater. Res. Soc. Symp. Proc. 1986, 55, 281.

[396] L. Li, A. Lundkvist, D. Andersson, U. Wilhelmsson, N. Nagai, A. C. Pardo, C. Nodin, A. Stahlberg, K. Aprico, K. Larsson, T. Yabe, L. Moons, A. Fotheringham, I. Davies, P. Carmeliet, J. P. Schwartz, M. Pekna, M. Kubista, F. Blomstrand, N. Maragakis, M. Nilsson, M. Pekny, J. Cereb. Blood Flow Metab. 2007, 28, 468.

[397] T. Nyberg, A. Shimada, K. Torimitsu, J. Neurosci. Methods 2007, 160, 16.

[398] W. R. Stauffer, X. T. Cui, Biomaterials 2006, 27, 2405.

[399] N. Gomez, C. E. Schmidt, J. Biomed. Mater. Res, Part A 2007, 81A, 135.

[400] Z. Zhang, M. Rouabhia, Z. Wang, C. Roberge, G. Shi, P. Roche, J. Li, L. H. Dao, Artif. Organs 2007, 31, 13.

[401] C. E. Schmidt, V. R. Shastri, J. P. Vacanti, R. Langer, PNAS 1997, 94, 8948.

[402] X. Cui, J. Wiler, M. Dzaman, R. A. Altschuler, D. C. Martin, Biomaterials 2003, 24, 777.

[403] E. M. Kelliher, T. L. Rose, Mater. Res. Soc. Symp. Proc. 1988, 110, 23.

[404] X. Cui, V. A. Lee, Y. Raphael, J. A. Wiler, J. F. Hetke, D. J. Anderson, D. C. Martin, J. Biomed. Mater. Res. 2001, 56, 261.

[405] X. Y. Cui, D. C. Martin, Sens. Actuators B-Chem. 2003, 89, 92.

[406] R. Wadhwa, C. F. Lagenaur, X. T. Cui, J. Control. Release 2006, 110, 531.

[407] G. R. Dieckman, A. B. Dalton, P. A. Johnson, J. Razal, J. Chen, G. M. Giordano, E. Munoz, I. H. Musselman, R. H. Baughman, R. K. Draper, J. Am. Chem. Soc. 2003, 125, 1770.

[408] E. Katz, I. Willner, Angew. Chem. Int. Ed. 2004, 43, 6042.

[409] F. Balavoine, P. Schultz, C. Richard, V. Mallouh, T. W. Ebbesen, C. Mioskowski, Angew. Chem. Int. Ed. 1999, 38, 1912.

[410] R. J. Chen, S. Bangsaruntip, K. A. Drouvalakis, N. W. S. Kam, M. Shim, Y. M. Li, W. Kim, P. J. Utz, H. J. Dai, Proc. Nat. Acad. Sci. USA 2003, 100, 4984.

[411] F. Patolsky, G. Zheng, O. Hayden, M. Lakadamyali, X. Zhuang, C. M. Lieber, Proc. Natl. Acad. Sci. USA 2004, 101, 14017.

[412] Z. Guo, P. J. Sadler, S. C. Tsang, Adv. Mater. 1998, 10, 701.

[413] S. C. Tsang, Z. Guo, Y. K. Chen, M. L. H. Green, H. A. O. Hill, T. W. Hambley, P. J. Sadler, Angew. Chem. Int. Ed. 1997, 36, 2198.

[414] S. Li, P. He, J. Dong, Z. Guo, L. Dai, J. Am. Chem. Soc. 2005, 127, 14.

[415] S. Hampel, D. Kunze, D. Haase, K. Kaermer, M. Rauschenbach, M. Ritschel, A. Leonhardt, J. Thomas, S. Oswald, V. Hoffmann, B. Buechner, Nanomedicine 2008, 3, 175.

[416] S. M. Garg, V. A. Deshmukh, Nanosci. Nanotechnol. 2007, 1, 45.

[417] S. Prakash, A. G. Kulamarva, Recent Pat. Drug Delivery Formulation 2007, 1, 214.

[418] Z. Liu, X. Sun, N. Nakayama-Ratchford, H. Dai, ACS Nano 2007, 1, 50.

[419] K. Kostarelos, L. Lacerda, G. Pastorin, W. Wu, S. Wieckowski, J. Luangsivilay, S. Godefroy, D. Pantarotto, J.-P. Briand, S. Muller, M. Prato, A. Bianco, Nat. Nanotechnol. 2007, 2, 108.

[420] E. W. Keefer, B. Botternan, M. I. Romero, A. F. Rossi, G. W. Gross, Nat. Nanotechnol. 2008, 3, 434.

[421] M. Olek, J. Ostrander, S. Jurga, H. Moehwald, N. Kotov, K. Kempa, M. Giersig, Nano Lett. 2004, 4, 1889.

[422] B. Shim, N. A. Kotov, Langmuir 2005, 21, 9381.
[423] B. S. Shim, Z. Tang, M. P. Morabito, A. Agarwal, H. Hong, N. A. Kotov, Chem. Mater. 2007, 19, 5467.

[424] T. Gabay, M. Ben-David, I. Kalifa, R. Sorkin, Z. R. Abrams, E. Ben-Jacob, Y. Hanein, Nanotechnology 2007, 18, 035201.

[425] T. Gabay, E. Jakobs, E. Ben-Jacob, Y. Hanein, Physica A 2005, 350 611.

[426] P. G. Whitten, A. A. Gestos, G. M. Spinks, K. J. Gilmore, G. G. Wallace, J. Biomed. Mater. Res. B Appl. Biomater. 2006, 828, 37.

[427] P. Fromherz, A. Offenhausser, T. Vetter, J. Weis, Science 1991, 252, 1290.

[428] T. D. Parsons, D. Kleinfeld, F. Raccuiabehling, B. M. Salzberg, Biophys. J. 1989, 56, 213.

[429] A. Offenhausser, C. Sprossler, M. Matsuzawa, W. Knoll, Biosens. Bioelectron. 1997, 12, 819.

[430] E. Neher, B. Sakmann, Nature 1976, 260, 799.

[431] G. Hans-Joachim, Angew. Chem. Int. Ed. Eng. 1992, 31, 45.

[432] P. Fromherz, A. Stett, Phys. Rev. Lett. 1995, 75, 1670.

[433] K. Wise, J. Angell, A microprobe with integrated amplifiers for neurophysiology, presented at Solid-State Circuits Conference. Digest of Technical Papers. 1971 IEEE International, 1971.

[434] K. Najafi, K. D. Wise, IEEE J. Solid-St. Circ. 1986, 21, 1035.

[435] W. L. C. Rutten, H. J. van Wier, J. H. M. Put, IEEE Trans. Biomed. Eng. 1991, 38, 192.

[436] K. Changhyun, K. D. Wise, IEEE J. Solid-St. Circ. 1996, 31, 1230.

[437] S. M. Yoon, E. Tokumitsu, H. Ishiwara, IEEE Trans. Electron Dev. 2000, 47, 1630.

[438] J. Pine, J. Neurosci. Meth. 1980, 2, 19.

[439] W. G. Regehr, J. Pine, D. B. Rutledge, IEEE Trans. Biomed. Eng. 1988, 35, 1023.

[440] G. Zeck, P. Fromherz, Proc. Natl. Acad. Sci. 2001, 98, 10457.

[441] S. Vassanelli, P. Fromherz, Appl. Phys. A: Mater. Sci. Process. 1998, 66, 459.

[442] M. Voelker, P. Fromherz, Small 2005, 1, 206.

[443] P. Bonifazi, P. Fromherz, Adv. Mater. 2002, 14, 1190.

[444] M. Ulbrich, P. Fromherz, Adv. Mater. 2001, 13, 344.

[445] M. Merz, P. Fromherz, Adv. Funct. Mater. 2005, 15, 739.

[446] A. Lambacher, M. Jenkner, M. Merz, B. Eversmann, R. A. Kaul, F. Hofmann, R. Thewes, P. Fromherz, Appl. Phys. A: Mater. Sci. Process. 2004, 79, 1607.

[447] M. Hutzler, A. Lambacher, B. Eversmann, M. Jenkner, R. Thewes, P. Fromherz, J. Neurophysiol. 2006, 96, 1638.

[448] R. A. Kaul, N. I. Syed, P. Fromherz, Phys. Rev. Lett. 2004, 92, 038102.

[449] Y. Cui, Q. Wei, H. Park, C. M. Lieber, Science 2001, 293, 1289.

[450] J. Hahm, C. M. Lieber, Nano Lett. 2004, 4, 51.

[45 1] J. T. Hu, T. W. Odom, C. M. Lieber, Acc. Chem. Res. 1999, 32, 435.

[452] E. Stern, J. F. Klemic, D. A. Routenberg, P. N. Wyrembak, D. B. Turner-Evans, A. D. Hamilton, D. A. LaVan, T. M. Fahmy, M. A. Reed, Nature 2007, 445, 519

[453] G. Zheng, F. Patolsky, Y. Cui, W. U. Wang, C. M. Lieber, Nat. Biotechnol. 2005, 23, 1294

[454] W. Sant, M. L. Pourciel-Gouzy, J. Launay, T. D. Conto, R. A. Colin, S. Martinez, P. Temple-Boyer, Sens. Actuators B-Chem. 2004, 103, 260.

[455] M. J. Schőning, A. Poghossian, Analyst 2002, 127, 1137.

[456] A. P. Soldatkin, J. Montoriol, W. Sant, C. Martelet, N. Jaffrezic-Renault, Biosens. Bioelectron. 2003, 19, 131.

[457] S. A. Campbell, The Science and Engineering of Microelectronic Fabrication Oxford University Press, New York, NY 1996.

[458] A. Cohen, J. Shappir, S. Yitzchiak, M. E. Spira, Biosens. Bioelectron. 2006 22, 656 .

[459] A. Cohen, M. E. Spira, S. Yitshaik, G. Borghs, O. Shwartzglass, J. Shappir, Biosens. Bioelectron. 2004, 19, 1703.

[460] P. Fromherz, Chem. Phys. Chem. 2002, 3, 276.

[461] R. Weis, B. Mueller, P. Fromherz, Phys. Rev. Lett. 1996, 76, 327.

[462] G. Wrobel, R. Seifert, S. Ingebrandt, J. Enderlein, H. Ecken, A. Baumann, U. B. Kaupp, A. Offenhäusser, Biophys. J. 2005, 89, 3628. 
[463] X. Zhou, J. Moran-Mirabal, M. G. Craighead, P. L. McEuen, Nat. Nanotech. 2007, 2, 185

[464] N. J. Sniadecki, R. A. Desai, S. A. Ruiz, C. S. Chen, Ann. Biomed. Eng. 2006, $34,59$.

[465] A. Javey, S. Nam, R. S. Friedman, H. Yan, C. M. Lieber, Nano Lett. 2007, 7, 773.

[466] D. Whang, S. Jin, Y. Wu, C. M. Lieber, Nano Lett. 2003, 3, 1255.

[467] I. Schoen, P. Fromherz, Biophys. J. 2007, 92, 1096.

[468] M. Tarek, Biophys. J. 2005, 88, 4045.

[469] E. Steidl, E. Neveu, D. Bertrand, B. Buisson, Brain Res. 2006, 1096, 70.

[470] A. T. Gulledge, G. J. Stuart, J. Neurosci. 2003, 23, 11363.

[471] M. E. Larkum, J. J. Zhu, J. Neurosci. 2002, 22, 6991.

[472] U. Windhorst, H. Johansson, Modern Techniques in Neuroscience Research: Electrical Activity of Individual Neurons In Situ: Extra- and Intracellular, Springer, New York, NY 1999.

[473] L. G. Nowak, J. Bullier, Exp. Brain Res. 1998, 118, 477.

[474] G. J. Stuart, B. Sakmann, Nature. 1994, 367, 69.

[475] M. Zochowski, M. Wachowiak, C. X. Falk, L. B. Cohen, Y.-W. Lam, S. Antic, D. Zecevic, Biol. Bull. 2000, 198, 1.

[476] N. Bhadra, K. L. Kilgore, IEEE Trans. Neural Syst. Rehabil. 2004, 12, 313.

[477] K. L. Casey, M. Blick, Brain Res. 1969, 13, 155.

[478] M. Sassen, M. Zimmermann, Pfleugers Arch. 1973, 341, 179.

[479] N. Gomez, J. O. Winter, F. Shieh, A. E. Saunders, B. A. Korgel, C. E. Schmidt, Talanta 2005, 67, 462.

[480] M. Frydrych, P. Silfsten, S. Parkkinen, J. Parkkinen, T. Jaaskelainen, BioSystems 2000, 54, 131.

[481] T. Yamashita, T. Shima, Y. Nishizaki, M. Kimura, H. Hara, S. Inoue, Jpn. J. Appl. Phys. 2008, 47, 1924.

[482] M. Kimura, T. Shima, K. Yamashita, Y. Uraoka, Application: JP Patent 2006-262746, 2008079799 2008

[483] E. Funatsu, Y. Nitta, J.-Y. Tanaka, K. Kyuma, Jpn. J. Appl. Phys, Part 2 1999, 38, L938.

[484] W. Roush, Science 1995, 268, 637.

[485] J.-M. Seo, S. J. Kim, H. Chung, E. T. Kim, H. G. Yu, Y. S. Yu, Mater. Sci. Eng. C 2004, C24, 185.

[486] E. M. Callaway, R. Yuste, Curr. Opin. Neurobiol. 2002, 12, 587.

[487] H. Bayley, Nat. Chem. Biol. 2006, 2, 11.

[488] B. V. Zemelman, G. A. Lee, M. Ng, G. Miesenbock, Neuron 2002, 33, 15.

[489] S. Q. Lima, G. Miesenboeck, Cell 2005, 121, 141.

[490] V. Nikolenko, K. E. Poskanzer, R. Yuste, Nat. Methods 2007, 4, 943.

[491] F. Zhang, L.-P. Wang, M. Brauner, J. F. Liewald, K. Kay, N. Watzke, P. G. Wood, E. Bamberg, G. Nagel, A. Gottschalk, K. Deisseroth, Nature 2007, 446, 633.

[492] V. Gradinaru, K. R. Thompson, F. Zhang, M. Mogri, K. Kay, M. B. Schneider, K. Deisseroth, J. Neurosci. 2007, 27, 14231.

[493] R. D. Airan, E. S. Hu, R. Vijaykumar, M. Roy, L. A. Meltzer, K. Deisseroth, Curr. Opin. Neurobiol. 2007, 17, 587.

[494] R. D. Airan, L. A. Meltzer, M. Roy, Y. Gong, H. Chen, K. Deisseroth, Science 2007, 317, 819.

[495] J. O. Winter, T. Y. Liu, B. A. Korgel, C. E. Schmidt, Adv. Mater. 2001, 13, 1673.

[496] T. Cassagneau, T. E. Mallouk, J. H. Fendler, J. Am. Chem. Soc. 1998, 120, 7848

[497] C. Tedeschi, H. Moehwald, S. Kirstein, J. Am. Chem. Soc. 2001, 123, 954.

[498] M. Durstock, M. Rubner, Langmuir 2001, 17, 7865.
[499] Y. Zhong, R. V. Bellamkonda, J. Control. Release 2005, 106, 309

[500] W. He, G. C. McConnell, T. M. Schneider, R. V. Bellamkonda, Adv. Mater. 2007, 19, 3529.

[501] B. E. Gliner, Application: US Patent 2002-112301, 2003 187490, 2003.

[502] Z. Hu, D. M. Zhou, R. Greenberg, T. Thundat, Biomaterials 2006, 27, 2009.

[503] A. Hung, D. Zhou, R. Greenberg, J. W. Judy, in Proc. Electrochem. Soc., Microfabricated Systems and MEMS VII, PV2004-09 (Eds: J. L. Davidson, P. J. Hesketh, D. Misra, E. Shoji), ECS, New Jersey 2004, 72-80.

[504] I. Manna, W. M. Steen, K. G. Watkins, Surf. Eng.: Mater. Sci. I, Proc. Symp. 2000, p. 377.

[505] K. G. Watkins, W. M. Steen, I. Manna, D. F. Williams, S. Rhodes, P. Mazzoldi, S. Lo Russo, M. G. S. Ferreira, J. T. Rito, T. Moura-Silva, A. M. P. Simoes, Laser Inst. Am. (Publ.) 1996, 81, A37.

[506] X. Y. Cui, V. A. Lee, Y. Raphael, J. A. Wiler, J. F. Hetke, D. J. Anderson, D. C. Martin, J. Biomed. Mater. Res. 2001, 56, 261.

[507] A. A. Mamedov, N. A. Kotov, M. Prato, D. Guldi, J. Wicksted, A. Hirsch, Nat. Mater. 2002, 1, 190.

[508] F. Patolsky, B. P. Timko, G. F. Zheng, C. M. Lieber, MRS Bulletin 2007, 32, 142.

[509] A. Agarwal, S. W. Huang, M. O'Donnell, K. C. Day, M. Day, N. Kotov, S. Ashkenazi, J. Appl. Phys. 2007, 102, 064701/1.

[510] G. L. Fain, Molecular and Cellular Physiology of Neurons, Harvard University Press, Cambridge, MA 1999.

[511] M. London, M. Häusser, Annu. Rev. Neurosci. 2005, 28, 503.

[512] F. Hofmann, E. Guenther, H. Hämmerle, C. Liebrock, V. Berezin, E. Bock, H. Volkmer, Brain Res. 2004, 1071, 184.

[513] R. Genov, M. Stanacevic, M. Naware, G. Cauwenberghs, N. V. Thakor, IEEE. Trans. Circ. -I 2006, 53, 2371.

[514] A. Stett, U. Egert, E. Guenther, F. Hofmann, T. Meyer, W. Nisch, H. Haemmerle, Anal. Bioanal. Chem. 2003, 377, 486.

[515] S. Joo, R. B. Brown, Chem. Rev. 2008, 108, 638.

[516] P. Estrela, A. G. Stewart, S. D. Keighley, P. Migliorato, J. Korean Phys. Soc. 2006, 48, S22.

[517] H. E. Katz, Electroanalysis 2004, 16, 1837.

[518] A. Bachtold, P. Hadley, T. Nakanishi, C. Dekker, Science 2001, 294, 1317. [519] A. Javey, J. Guo, Q. Wang, M. Lundstrom, H. J. Dai, Nature 2003, 424, 654.

[520] E. S. Snow, F. K. Perkins, E. J. Houser, S. C. Badescu, T. L. Reinecke, Science 2005, 307, 1942

[521] B. L. Allen, P. D. Kichambare, A. Star, Adv. Mater. 2007, 19, 1439.

[522] P. Avouris, J. Chen, Mater. Today 2006, 9, 46.

[523] G. Gruner, Anal. Bioanal. Chem. 2006, 384, 322.

[524] J. Lee, M. J. Cuddihy, N. A. Kotov, Tissue Eng, Part B 2008, 14, 61.

[525] J. Lee, S. Shanbhag, N. A. Kotov, J. Mater. Chem. 2006, 16, 3558.

[526] G. Martino, S. Pluchino, Nat. Rev. Neurosci. 2006, 7, 395.

[527] H.-J. Song, C. F. Stevens, F. H. Gage, Nat. Neurosci. 2002, 5, 438

[528] J. Ban, P. Bonifazi, G. Pinato, F. D. Broccard, L. Studer, V. Torre, M. E. Ruaro, Stem Cells 2007, 25, 738.

[529] L. Bai, S. L. Gerson, R. H. Miller, in The Cell Cycle in the Central Nervous System (Ed: D. Janigro), Humana Press, Totowa, NJ 2006, 511.

[530] Y. M. Elcin, Adv. Exp. Med. Biol. 2004, 553, 301.

[531] R. L. Gardner, C. R. Biol. 2007, 330, 465

[532] S. H. Hwang, M. H. Kim, I. H. Yang, J. Y. Bahk, H. Han, Biotechnol. Bioprocess Eng. 2007, 12, 32.

[533] J. J. Ross, C. M. Verfaillie, Philos. Trans. R. Soc, B 2008, 363, 199. 